\title{
QUANTUM UNIQUE ERGODICITY OF DEGENERATE EISENSTEIN SERIES ON $G L(n)$
}

\author{
LIYANG ZHANG
}

\begin{abstract}
We prove quantum unique ergodicity for a subspace of the continuous spectrum spanned by the degenerate Eisenstein Series on $G L(n)$.
\end{abstract}

\section{CONTENTS}

1. Introduction

1.1. Introduction

1.2. Strategy for proof of Theorem 1.1

2. Automorphic Forms on $G L(n)$

2.1. Automorphic Functions, Automorphic Forms, and Fourier Expansion 4

2.2. Parabolic Subgroups and Eisenstein Series

2.3. Incomplete Eisenstein Series and Spectral Decomposition

3. Fourier Expansion of Degenerate Eisenstein Series

3.1. Fourier Expansion of Degenerate Eisenstein Series

3.2. Constant Term Computation

4. Cuspidal and Non-Minimal Eisenstein Contribution 19

4.1. Some Basic Lemmata 19

4.2. Partition of the Form $n=n_{1}+\cdots+n_{r-1}+1 \quad 20$

4.3. A $G L(2)$ Calculation 26

4.4. Cuspidal Contribution 27

4.5. Partition of type $n=n_{1}+\cdots+n_{r}, n_{r} \geq 2 . \quad 29$

5. Main Term From Minimal Parabolic Contribution 32

References

\section{INTRODUCTION}

1.1. Introduction. In the classical setting, the evolution of a dynamical system $(X, \mu, T)$ can be described by the geodesic flow:

$$
g_{t}: T^{*} X \rightarrow T^{*} X
$$

where $g_{t} \mathbf{x}_{0}=\mathbf{x}_{t}$ is given by the Hamiltonian. We say the system is ergodic if for every $f \in L_{\mu}^{2}$ and for almost every starting position $\mathbf{x}_{0}$ the time average becomes the spatial average:

$$
\lim _{S \rightarrow \infty} \frac{1}{S} \int_{0}^{S} f\left(g_{t}\left(\mathbf{x}_{0}\right)\right) d t=\frac{1}{\mu(X)} \int_{X} f(\mathbf{x}) d \mathbf{x}
$$

A classic example of an ergodic dynamical system is the Bunimovich Stadium [Bu][BS].

\footnotetext{
Key words and phrases. Quantum Unique Ergodicity, $G L(n)$ Eisenstein Series, Incomplete Eisenstein Series,

The author was supported by the following grants from Alex Kontorovich: NSF CAREER grant DMS-1254788 and DMS1455705 .
} 
In the quantum setting, the evolution of a system is governed by the Schrödinger equation:

$$
-\frac{\hbar^{2}}{2 m} \Delta \psi_{n}=\lambda_{n} \psi_{n}
$$

where $\Delta$ is the Laplacian. A system is quantum uniquely ergodic if in the semiclassical limit $(\hbar \rightarrow 0)$, each individual eigenfunction $\left|\psi_{n}\right|^{2}$ become equi-distributed.

Let $(M, \mu)$ be a Riemannian manifold with laplacian $\Delta$ and let $\psi_{n}$ a set of orthonormal eigenfunctions of $\Delta$ with corresponding eigenvalues $0 \leq \lambda_{1} \leq \lambda_{2} \leq \ldots$ Schnirelman [Sh], Colin de Verdiére [Co] and Zelditch [Ze1] proved quantum ergodicity: eigenfunctions of $\Delta$ become equi-distributed with respect to the volume measure in the high energy limit along a subsequence $n_{k}$ of density one. Zelditch [Ze2] extended this result to the modular surface, which is not compact. Hejhal-Rackner [HR], Rudnick-Sarnak [RS] conjectured that there are no exceptional subsequences, that is, there is quantum unique ergodicity. The arithmetic version of the conjecture was famously proved by Lindenstrauss [Li] and Soundararajan [S]. For higher rank, Silberman and Venkatesh formualted and proved some cases of quantum unique ergodicity for compact locally symmetric spaces in [SV1] and [SV2].

For non-compact quotients, the Laplacian $\Delta$ has continuous spectrum and one can formulate QUE for such. For example, on the modular surface the continuous spectrum is spanned by the Eisenstein series

$$
E(z, s)=\sum_{\gamma \in \Gamma_{\infty} \backslash S L(2, \mathbb{Z})} \Im(\gamma z)^{s} .
$$

Arithmetic quantum unique ergodicity of Eisenstein series on $G L(2)$ was first formulated and proved by Luo and Sarnak in [LS]. The unitary Eisenstein series $E\left(z, \frac{1}{2}+i t\right)$ is an eigenfunction of the hyperbolic Laplacian with eigenvalue $\frac{1}{4}+t^{2}$. Define $d \nu_{t}=\left|E\left(z, \frac{1}{2}+i t\right)\right|^{2} \frac{d x d y}{y^{2}}$. Then for compact Jordan measurable sets $A, B$ in $S L(2, \mathbb{Z}) \backslash S L(2, \mathbb{R}) / S O(2, \mathbb{R})$, Luo and Sarnak showed

$$
\lim _{t \rightarrow \infty} \frac{\nu_{t}(A)}{\nu_{t}(B)}=\frac{\operatorname{Vol}(A)}{\operatorname{Vol}(B)}
$$

which represents arithmetic quantum unique ergodicity for the continuous spectrum. More precisely, they showed

$$
\nu_{t}(A)=\frac{12}{\pi} \operatorname{Vol}(A) \log t+O(1)
$$

as $t \rightarrow \infty$. Note that ergodic methods typically do not give rates. We also remark that the constant $\frac{12}{\pi}$ is different from that in [LS] because of different normalization.

In this paper, we study the analog of Luo and Sarnak's result on a subspace of the $G L(n)$ continuous spectrum spanned by the degenerate Eisenstein series induced from the maximal parabolic subgroup with the constant function $E_{n-1,1}(z, s, 1)$. Eisenstein series are eigenfunctions of Casimir operators and on $G L(n)$, the analog of the hyperbolic Laplacian is $\Delta_{2, n}$ (see section 2) where

$$
\left(\Delta_{2, n}+\left(\frac{n^{2}-n}{8}+\frac{n^{2}-n}{2} t^{2}\right)\right) E_{n-1,1}\left(z, \frac{1}{2}+i t, 1\right)=0
$$

Define $\mu_{n, t}=\left|E_{n-1,1}\left(z, \frac{1}{2}+i t, 1\right)\right|^{2} d^{*} z$ where $d^{*} z$ is the Haar measure. We will show the following:

Theorem 1.1. Let $A, B$ be compact Jordan measurable subsets of $S L(n, \mathbb{Z}) \backslash S L(n, \mathbb{R}) / S O(n, \mathbb{R})$. Then for $n \geq 2$,

$$
\lim _{t \rightarrow \infty} \frac{\mu_{n, t}(A)}{\mu_{n, t}(B)}=\frac{\operatorname{Vol}(A)}{\operatorname{Vol}(B)}
$$

More precisely,

$$
\mu_{n, t}(A)=\frac{2}{\xi(n)} \operatorname{Vol}(A) \log t+O_{A}(1)
$$


as $t \rightarrow \infty$.

Remark 1.1. This theorem will serve as a stepping stone towards proving quantum unique ergodicity of higher rank non-degenerate Eisenstein series. QUE of higher rank non-degenerate Eisenstein series is a much more difficult problem as it is related to the shifted convolution problem involving generalized divisor functions and Fourier coefficients of higher rank Maass cusp forms.

1.2. Strategy for proof of Theorem 1.1. We use the spectral decomposition of $\mathfrak{L}^{2}\left(S L_{n}(\mathbb{Z}) \backslash X_{n}\right)$ to divide the proof of Theorem 1.1 into evaluating the following three type of integrals (see Theorem 2.2):

$$
\begin{aligned}
& \int_{S L_{n}(\mathbb{Z}) \backslash X_{n}} \phi(z)\left|E_{n-1,1}\left(z, \frac{1}{2}+i t, 1\right)\right|^{2} d^{*} z \\
& \int_{S L_{n}(\mathbb{Z}) \backslash X_{n}} E_{1, \ldots, 1}(z, \eta)\left|E_{n-1,1}\left(z, \frac{1}{2}+i t, 1\right)\right|^{2} d^{*} z \\
& \int_{S L_{n}(\mathbb{Z}) \backslash X_{n}} E_{n_{1}, \ldots, n_{r}}\left(z, \psi, u_{1}, \ldots, u_{r}\right)\left|E_{n-1,1}\left(z, \frac{1}{2}+i t, 1\right)\right|^{2} d^{*} z .
\end{aligned}
$$

Here $\phi(z)$ is a $G L(n)$ cusp form, $E_{1, \ldots, 1}(z, \eta)$ is an incomplete Eisenstein series associated to the minimal parabolic subgroup, and $E_{n_{1}, \ldots, n_{r}}\left(z, \psi, u_{1}, \ldots, u_{r}\right)$ is an incomplete Eisenstein series associated to the $\left(n_{1}, \ldots, n_{r}\right)$ parabolic subgroup induced from cusp forms $u_{j}$ on $G L\left(n_{j}\right)$. These integrals will be evaluated in sections 4 and 5 .

Acknowledgement. I would like to thank Alex Kontorovich for suggesting the problem, many enlightening discussions, and careful readings of the various versions of this paper. I also would like to thank Valentin Blomer for helpful comments. 


\section{Automorphic Forms on $G L(n)$}

Here we give a brief introduction to automorphic forms on $G L(n)$, make some preliminary computations and set the notation for the rest of the paper.

2.1. Automorphic Functions, Automorphic Forms, and Fourier Expansion. We are working over the generalized upper half space $X_{n}:=G L_{n}(\mathbb{R}) /\left(O_{n}(\mathbb{R}) \cdot \mathbb{R}^{*}\right)$. By the Iwasawa decomposition (see section 1.2 of $[\mathrm{Go}]), X_{n}$ consists of matrices of the form $z=x \cdot y$ with

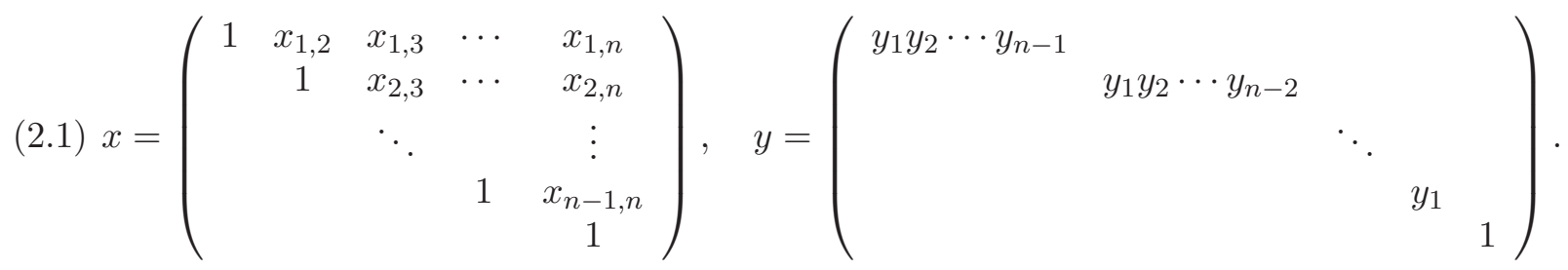

where $x_{i, j} \in \mathbb{R}$ and $y_{k}>0$. We say a function $f$ is automorphic if

$$
f(\gamma z)=f(z) \quad \forall \gamma \in S L_{n}(\mathbb{Z}), \forall z \in X_{n} .
$$

The space $X_{n}$ is equipped with a left $G L_{n}(\mathbb{R})$-invariant measure $d^{*} z$ on $X_{n}$ given explicitly by

$$
d^{*} z=c_{n} d^{*} x d^{*} y
$$

where

$$
c_{n}=n^{-1} \prod_{\ell=2}^{n} \xi(\ell)^{-1}, \quad d^{*} x=\prod_{1 \leq i<j \leq n} d x_{i, j}, \quad \prod_{k=1}^{n-1} d_{k}^{-k(n-k)} \frac{d y_{k}}{y_{k}} .
$$

Throughout this paper, we define the completed zeta-function $\xi(s)$ to be $\pi^{-\frac{s}{2}} \Gamma\left(\frac{s}{2}\right) \zeta(s)$. The normalization for this measure is chosen so that

$$
\int_{S L_{n}(\mathbb{Z}) \backslash X_{n}} 1 d^{*} z=1
$$

Our interest lies in the Hilbert space $\mathfrak{L}\left(S L_{n}(\mathbb{Z}) \backslash X_{n}\right)$ where the inner product is defined by

$$
\langle f, g\rangle=\int_{S L_{n}(\mathbb{Z}) \backslash X_{n}} f(z) \bar{g}(z) d^{*} z .
$$

For a smooth function $f$ in $\mathfrak{L}\left(S L_{n}(\mathbb{Z}) \backslash X_{n}\right)$, standard Fourier theory with the automorphy of $f$ give rise to a Fourier expansion of $f$.

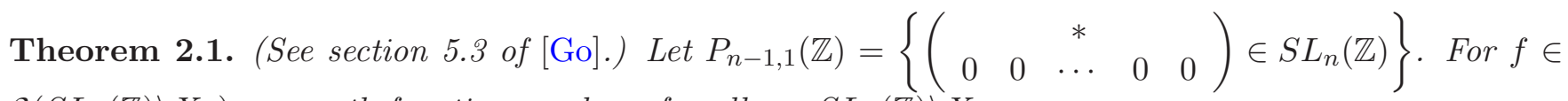
$\mathfrak{L}\left(S L_{n}(\mathbb{Z}) \backslash X_{n}\right)$ a smooth function, we have for all $z \in S L_{n}(\mathbb{Z}) \backslash X_{n}$

$$
f(z)=\left.\sum_{m_{1}=0}^{\infty} \sum_{m_{2}=0}^{\infty} \sum_{\gamma_{2} \in P_{1,1} \backslash S L_{2}(\mathbb{Z})}^{\prime} \cdots \sum_{m_{n-1}=0}^{\infty} \sum_{\gamma_{n-1} \in P_{n-2,1} \backslash S L_{n-1}(\mathbb{Z})}^{\prime} \hat{f}_{\left(m_{1}, \ldots, m_{n-1}\right)}(z)\right|_{\gamma_{2} \cdots \gamma_{n-1}} .
$$

where the slash operator is defined by

$$
\left.g(z)\right|_{\gamma_{2} \cdots \gamma_{n-1}}=g\left(\left(\begin{array}{cc}
\gamma_{2} & \\
& I_{n-2}
\end{array}\right) \cdots\left(\begin{array}{cc}
\gamma_{n-1} & \\
& 1
\end{array}\right) \cdot z\right)
$$


and for each $2 \leq h \leq n-1$, the primed summation over each $P_{h-1,1} \backslash S L_{h}(\mathbb{Z})$ is summed only if $m_{h} \neq 0$. Let $U_{n}(\mathbb{Z})\left(U_{n}(\mathbb{R})\right)$ denote the group of $n \times n$ upper triangular matrices with integer (real) entries and 1 's on the diagonal

$$
\hat{f}_{\left(m_{1}, \ldots, m_{n-1}\right)}(z)=\int_{0}^{1} \cdots \int_{0}^{1} \phi(u \cdot z) e\left(-m_{1} u_{1,2}-m_{2} u_{2,3}-\cdots-m_{n-1} u_{n-1, n}\right) \prod_{1 \leq i<j \leq n} d u_{i, j}
$$

and

$$
u=\left(\begin{array}{ccccc}
1 & u_{1,2} & u_{1,3} & \cdots & u_{1, n} \\
& 1 & u_{2,3} & \cdots & u_{2, n} \\
& & \ddots & & \vdots \\
& & & 1 & u_{n-1, n} \\
& & & & 1
\end{array}\right) \in U_{n}(\mathbb{R}) .
$$

Our goal is to describe a spectral decomposition of the space $\mathfrak{L}\left(S L_{n}(\mathbb{Z}) \backslash X_{n}\right)$. It is natural to turn to the theory of differential operators. Let $\mathfrak{D}_{n}$ be the center of the universal enveloping algebra of the Lie algebra $\mathfrak{g l}_{n}(\mathbb{R})$. Let $E_{i, j} \in \mathfrak{g l}_{n}(\mathbb{R})$ denote the matrix with a 1 at the $(i, j)$ entry and zeros elsewhere. We define the differential operator $D_{i, j}$ by

$$
\left(D_{i, j} f\right)(g)=\left.\frac{\partial}{\partial t} f\left(g \cdot \exp \left(t E_{i, j}\right)\right)\right|_{t=0}
$$

for a smooth function $f: G L_{n}(\mathbb{R}) \mapsto \mathbb{C}$.

Proposition 2.1. (See section 2.3 of [Go].) For $n \geq 2$ and $2 \leq m \leq n$, the differential operators (Casimir operators)

$$
\Delta_{m, n}:=\sum_{i_{1}=1}^{n} \sum_{i_{2}=1}^{n} \cdots \sum_{i_{m}=1}^{n} D_{i_{1}, i_{2}} \circ D_{i_{2}, i_{3}} \circ \cdots \circ D_{i_{m}, i_{1}}
$$

generate $\mathfrak{D}_{n}$ as a polynomial algebra of rank $n-1$.

For $n \geq 2$ and $\nu=\left(\nu_{1}, \ldots, \nu_{n-1}\right) \in \mathbb{C}^{n-1}$, the $I$-function defined by

$$
I_{\nu}(z)=\prod_{i=1}^{n-1} \prod_{j=1}^{n-1} y_{i}^{b_{i, j} \nu_{j}}
$$

where

$$
b_{i, j}= \begin{cases}i j & \text { if } i+j \leq n \\ (n-i)(n-j) & \text { if } i+j \geq n\end{cases}
$$

is an eigenfunction of all differential operators in $\mathfrak{D}_{n}$. Let $\lambda_{m, n}$ be the corresponding eigenvalues, i.e.

$$
\Delta_{n, m} I_{\nu}(z)=\lambda_{m, n} I_{\nu}(z) .
$$

Definition 2.1. An automorphic form $\phi(z) \in \mathcal{L}^{2}\left(S L_{n}(\mathbb{Z}) \backslash X_{n}\right)$ of spectral type $\nu \in \mathbb{C}^{n-1}$ is a smooth function satisfying:

(1) $\phi(\gamma z)=\phi(z), \quad \forall \gamma \in S L_{n}(\mathbb{Z}), \forall z \in X_{n}$,

(2) $\Delta_{m, n} \phi(z)=\lambda_{m, n} \phi(z)$;

if $\phi(z)$ also satisfies

$$
\int_{\left(S L_{n}(\mathbb{Z}) \cap U\right) \backslash U} \phi(u \cdot z) d u=0,
$$


for all matrices of the form

$$
U=\left\{\left(\begin{array}{ccccc}
I_{r_{1}} & & & & \\
& I_{r_{2}} & & * & \\
& & \ddots & & \\
& & & & I_{r_{m}}
\end{array}\right)\right\} \subset S L_{n}(\mathbb{R})
$$

with $r_{1}+\cdots+r_{m}=n$, then $\phi(z)$ is a Maass form.

For an automorphic form $\phi(z)$ of type $\nu$, the Fourier expansion (2.2) can be made more explicit by the theory of Whittaker functions. Multiplicity one theorem implies that each $\hat{\phi}_{\left(m_{1}, \ldots, m_{n-1}\right)}(z)$ can be expressed in terms of Whittaker functions:

$$
W_{\left(m_{1}, \ldots, m_{n-1}\right)}^{\nu}(z, w):=\int_{U_{n}(\mathbb{R})} I_{\nu}(w \cdot u \cdot z) e\left(-m_{1} u_{1,2}-\cdots-m_{n-1} u_{n-1, n}\right) \prod_{1 \leq i<j \leq n} d u_{i, j}
$$

where $w \in W_{n}$ is an element of the Weyl group. If $\phi(z)$ is a Maass cusp form, then (2.2) can be further simplified to

$$
\phi(z)=\left.\sum_{\gamma \in U_{n-1}(\mathbb{Z}) \backslash S L_{n-1}(\mathbb{Z})} \sum_{m_{1}=1}^{\infty} \cdots \sum_{m_{n-2}=1}^{\infty} \sum_{m_{n-1} \neq 0} a_{\left(m_{1}, \ldots, m_{n-1}\right)} W_{\left(m_{1}, \ldots, m_{n-1}\right)}^{\nu}\left(z, w_{l}\right)\right|_{\gamma},
$$

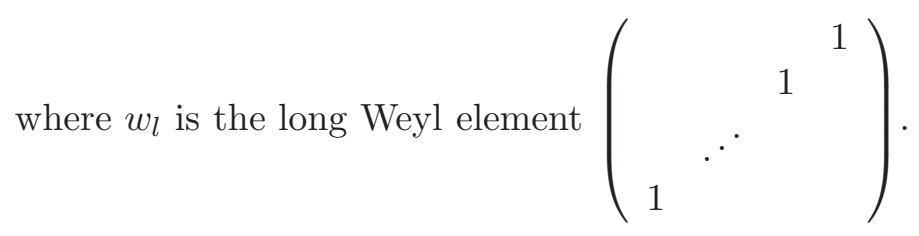

2.2. Parabolic Subgroups and Eisenstein Series. Here we give a summary of Langlands' theory of Eisenstein series associated to Maass forms.

Definition 2.2. The standard parabolic subgroup $P_{n_{1}, \ldots, n_{r}}(\mathbb{R}) \subset G L_{n}(\mathbb{R})$ associated to the partition $n=n_{1}+n_{2}+\cdots+n_{r}$ is defined to be the group of matrices of the form

$$
\left(\begin{array}{cccc}
\mathfrak{m}_{n_{1}} & * & \cdots & * \\
0 & \mathfrak{m}_{n_{2}} & \cdots & * \\
\vdots & \vdots & \ddots & \vdots \\
0 & 0 & \cdots & \mathfrak{m}_{n_{r}}
\end{array}\right)
$$

where $\mathfrak{m}_{n_{i}} \in G L_{n_{i}}(\mathbb{R})$ for $1 \leq i \leq r$. We also define $P_{n_{1}, \ldots, n_{r}}(\mathbb{Z})=P_{n_{1}, \ldots, n_{r}}(\mathbb{R}) \cap S L_{n}(\mathbb{Z})$. Two parabolic subgroups $P_{n_{1}, \ldots, n_{r}}(\mathbb{R}), P_{n_{1}^{\prime}, \ldots, n_{r}^{\prime}}(\mathbb{R})$ of $G L_{n}(\mathbb{R})$ are said to be associate if the set $\left\{n_{1}, \ldots, n_{r}\right\}$ is a permutation of the set $\left\{n_{1}^{\prime}, \ldots, n_{r}^{\prime}\right\}$.

A parabolic subgroup $P_{n_{1}, \ldots, n_{r}}(\mathbb{R})$ can be decomposed into

$$
P_{n_{1}, \ldots, n_{r}}(\mathbb{R})=N_{n_{1}, \ldots, n_{r}}(\mathbb{R}) M_{n_{1}, \ldots, n_{r}}(\mathbb{R}),
$$


where

$$
N_{n_{1}, \ldots, n_{r}}(\mathbb{R})=\left\{\left(\begin{array}{cccc}
I_{n_{1}} & * & \cdots & * \\
0 & I_{n_{2}} & \cdots & * \\
\vdots & \vdots & \ddots & \vdots \\
0 & 0 & \cdots & I_{n_{r}}
\end{array}\right) \in G L_{n}(\mathbb{R}), I_{k} \text { is an } k \times k \text { identity matrix }\right\}
$$

is the unipotent radical and

$$
M_{n_{1}, \ldots, n_{r}}(\mathbb{R})=\left\{\left(\begin{array}{cccc}
\mathfrak{m}_{n_{1}} & 0 & \cdots & 0 \\
0 & \mathfrak{m}_{n_{2}} & \cdots & 0 \\
\vdots & \vdots & \ddots & \vdots \\
0 & 0 & \cdots & \mathfrak{m}_{n_{r}}
\end{array}\right), \mathfrak{m}_{k} \in G L_{n_{k}}(\mathbb{R})\right\}
$$

is the Levi component. This is the Langlands decomposition of parabolic subgroups. We define $N_{n_{1}, \ldots, n_{r}}(\mathbb{Z})=$ $N_{n_{1}, \ldots, n_{r}}(\mathbb{R}) \cap S L_{n}(\mathbb{Z}), M_{n_{1}, \ldots, n_{r}}(\mathbb{Z})=M_{n_{1}, \ldots, n_{r}}(\mathbb{R}) \cap S L_{n}(\mathbb{Z})$. For $g \in P_{n_{1}, \ldots, n_{r}}(\mathbb{R})$, Langlands decomposition naturally gives rise to the projection maps $\mathfrak{m}_{n_{i}}: P_{n_{1}, \ldots, n_{r}}(\mathbb{R}) \mapsto G L_{n_{i}}(\mathbb{R})$ by

$$
g=\left(\begin{array}{cccc}
I_{n_{1}} & * & \cdots & * \\
0 & I_{n_{2}} & \cdots & * \\
\vdots & \vdots & \ddots & \vdots \\
0 & 0 & \cdots & I_{n_{r}}
\end{array}\right) \cdot\left(\begin{array}{cccc}
\mathfrak{m}_{n_{1}}(g) & 0 & \cdots & 0 \\
0 & \mathfrak{m}_{n_{2}}(g) & \cdots & 0 \\
\vdots & \vdots & \ddots & \vdots \\
0 & 0 & \cdots & \mathfrak{m}_{n_{r}}(g)
\end{array}\right)
$$

To describe Eisenstein series associated to Maass forms we also need to define $I$-functions associated to parabolic subgroups.

Definition 2.3. Let $s=\left(s_{1}, \ldots, s_{r}\right) \in \mathbb{C}^{r}$ satisfying $\sum_{i=1}^{r} n_{i} s_{i}=0$ and for $z \in X_{n}$ in Iwasawa form (2.1) we define

$$
I_{s}\left(z, P_{n_{1}, \ldots, n_{r}}\right)=\left(\prod_{j_{1}=n-n_{1}+1}^{n} Y_{j_{1}}\right)^{s_{1}} \cdot\left(\prod_{j_{2}=n-n_{1}-n_{2}+1}^{n-n_{1}} Y_{j_{2}}\right)^{s_{2}} \cdots\left(\prod_{j_{r}=1}^{n_{r}} Y_{j_{r}}\right)^{s_{r}}
$$

where $Y_{1}, Y_{2}, \ldots, Y_{n}$ are defined by

$$
\left(\begin{array}{ccccc}
Y_{n} & & & \\
& Y_{n-1} & & \\
& & \ddots & \\
& & & Y_{1}
\end{array}\right)=\left(\begin{array}{lllll}
y_{1} y_{2} \cdots y_{n-1} & & & \\
& y_{1} y_{2} \cdots y_{n-2} & & \\
& & \ddots & \\
& & & y_{1} & \\
& & & & 1
\end{array}\right) .
$$

Definition 2.4. Let $P_{n_{1}, \ldots, n_{r}}(\mathbb{R})$ be a parabolic subgroup of $G L_{n}(\mathbb{R})$ with projection maps $\mathfrak{m}_{n_{i}}$ defined by Langlands decomposition. Let $\phi_{i}$ be Maass forms on $G L_{n_{i}}(\mathbb{R})$ for $1 \leq i \leq r$, and let $s=\left(s_{1}, \ldots, s_{r}\right) \in \mathbb{C}^{r}$ satisfy

$$
\sum_{i=1}^{r} n_{i} s_{i}=0
$$

We define the Eisenstein series $E_{\left(n_{1}, \ldots, n_{r}\right)}\left(z, s, \phi_{1}, \ldots, \phi_{r}\right)$ by

$$
E_{\left(n_{1}, \ldots, n_{r}\right)}\left(z, s, \phi_{1}, \ldots, \phi_{r}\right)=\sum_{\gamma \in P_{n_{1}, \ldots, n_{r}}(\mathbb{Z}) \backslash S L_{n}(\mathbb{Z})} \prod_{i=1}^{r} \phi_{i}\left(\mathfrak{m}_{i}(\gamma z)\right) I_{s}\left(\gamma z, P_{n_{1}, \ldots, n_{r}}\right) .
$$

Because of the relation $\sum_{i=1}^{r} n_{i} s_{i}=0$, we may eliminate $s_{r}$. So $E_{n_{1}, \ldots, n_{r}}\left(z, s, \phi_{1}, \ldots, \phi_{r}\right)$ is really a function of $z$ and $s=\left(s_{1}, \ldots, s_{r-1}\right) \in \mathbb{C}^{r-1}$ and we will use this convention for the remaining part of this paper. 
We want to especially point out that if we use the partition $n=1+\cdots+1$, we get the minimal parabolic Eisenstein series

$$
E_{(1, \ldots, 1)}(z, s)=\sum_{\gamma \in P_{1, \ldots, 1}(\mathbb{Z}) \backslash S L_{n}(\mathbb{Z})} I_{s}(\gamma z) .
$$

For the maximal parabolic $P_{n-1,1}$, we can define the totally degenerate Eisenstein series

$$
E_{(n-1,1)}(z, s, 1)=\sum_{\gamma \in P_{n-1,1}(\mathbb{Z}) \backslash S L_{n}(\mathbb{Z})} I_{s}\left(\gamma z, P_{n-1,1}\right) .
$$

This is the main object of study in this paper.

Langlands' theory of constant terms along arbitrary parabolic subgroups play a central role in understanding these Eisenstein series. We will use this theory to understand the Fourier expansion of some Eisenstein series.

Proposition 2.2. Let $n=n_{1}+\ldots+n_{r}$ be a partition in descending order with $n_{r} \geq 2$, and let $E_{n_{1}, \ldots, n_{r}}\left(z, s, \phi_{1}, \ldots, \phi_{r}\right)$ be an Eisenstein series. Then its constant terms along $P_{(n-1,1)}, P_{(n-3,1,2)}, P_{(1, \ldots, 1)}$ are zero:

$$
\begin{array}{r}
\int_{N_{n-1,1}(\mathbb{Z}) \backslash N_{n-1,1}(\mathbb{R})} E_{\left(n_{1}, \ldots, n_{r}\right)}\left(z, s, \phi_{1}, \ldots, \phi_{r}\right)=0, \\
\int_{N_{n-3,1,2}(\mathbb{Z}) \backslash N_{n-3,1,2}(\mathbb{R})} E_{\left(n_{1}, \ldots, n_{r}\right)}\left(z, s, \phi_{1}, \ldots, \phi_{r}\right)=0, \\
\int_{N_{1, \ldots, 1}(\mathbb{Z}) \backslash N_{n-1,1}(\mathbb{R})} E_{\left(n_{1}, \ldots, n_{r}\right)}\left(z, s, \phi_{1}, \ldots, \phi_{r}\right)=0 .
\end{array}
$$

Proof. This is a direct result of the proposition in II.1.7 of [MW]. Since $n_{i} \geq 2$ for all $1 \leq i \leq r$, $w M_{n_{1}, \ldots, n_{r}}(\mathbb{R}) w^{-1}$ cannot be contained in either $M_{(n-1,1)}(\mathbb{R}), M_{(n-3,1,2)}(\mathbb{R})$ or $M_{(1, \ldots, 1)}(\mathbb{R})$ for any Weyl element $w$.

Corollary 2.1. Let $n=n_{1}+\ldots+n_{r}$ be a partition in descending order with $n_{r} \geq 2$, then in the Fourier expansion of $E_{\left(n_{1}, \ldots, n_{r}\right)}\left(z, s, \phi_{1}, \ldots, \phi_{r}\right)$, the Fourier coefficients $a_{\left(m_{1}, \ldots, m_{n-2}, 0\right)}, a_{\left(m_{1}, \ldots m_{n-4}, 0,0, m_{n-1}\right)}$ and $a_{(0, \ldots, 0)}$ are all zero. 
Proof. Let $f(z) E_{\left(n_{1}, \ldots, n_{r}\right)}\left(z, s, \phi_{1}, \ldots, \phi_{r}\right)$. We have

$$
\begin{aligned}
& \hat{f}_{\left(m_{1}, \ldots, m_{n-2}, 0\right)}(z)=\int_{0}^{1} \cdots \int_{0}^{1} f(u \cdot z) e\left(-m_{1} u_{1,2}-m_{2} u_{2,3}-\cdots-m_{n-2} u_{n-2, n-1}\right) \prod_{1 \leq i<j \leq n} d u_{i, j} \\
& =\int_{0}^{1} \cdots \int_{0}^{1} f\left(\left(\begin{array}{ccccc}
1 & 0 & 0 & \cdots & u_{1, n} \\
& 1 & 0 & \cdots & u_{2, n} \\
& & \ddots & \cdots & \vdots \\
& & & 1 & u_{n-1, n} \\
& & & & 1
\end{array}\right) \cdot\left(\begin{array}{ccccc}
1 & u_{1,2} & \cdots & u_{1, n-1} & 0 \\
& 1 & \cdots & u_{2, n-1} & 0 \\
& & \ddots & \vdots & \vdots \\
& & & 1 & 0 \\
& & & & 1
\end{array}\right) \cdot z \prod_{1 \leq i \leq n-1} d u_{i, n}\right. \\
& \times e\left(-m_{1} u_{1,2}-m_{2} u_{2,3}-\cdots-m_{n-2} u_{n-2, n-1}\right) \prod_{1 \leq i<j \leq n-1} d u_{i, j} \\
& =\int_{0}^{1} \cdots \int_{0}^{1} \int_{N_{n-1,1}(\mathbb{Z}) \backslash N_{n-1,1}(\mathbb{R})} f\left(u^{\prime} \cdot\left(\begin{array}{ccccc}
1 & u_{1,2} & \cdots & u_{1, n-1} & 0 \\
& 1 & \cdots & u_{2, n-1} & 0 \\
& & \ddots & \vdots & \vdots \\
& & & 1 & 0 \\
& & & & 1
\end{array}\right) \cdot z\right) \prod_{1 \leq i \leq n-1} d u_{i, n}^{\prime} \\
& \times e\left(-m_{1} u_{1,2}-m_{2} u_{2,3}-\cdots-m_{n-2} u_{n-2, n-1}\right) \prod_{1 \leq i<j \leq n-1} d u_{i, j} \\
& =0 \text {, }
\end{aligned}
$$

by Proposition 2.2. For $\hat{f}_{\left(m_{1}, \ldots m_{n-4}, 0,0, m_{n-1}\right)}$, the proof is similar as we have

$$
\begin{aligned}
& u=\left(\begin{array}{ccccccc}
1 & 0 & \cdots & 0 & u_{1, n-2} & u_{1, n-1} & u_{1, n}-u_{1, n-1} u_{n-1, n} \\
& 1 & \ddots & \vdots & u_{2, n-2} & u_{2, n-1} & u_{2, n}-u_{2, n-1} u_{n-1, n} \\
& & \ddots & 0 & \vdots & \vdots & \vdots \\
& & & 1 & u_{n-3, n-2} & u_{n-3, n-1} & u_{n-3, n}-u_{n-3, n-1} u_{n-1, n} \\
& & & & 1 & u_{n-2, n-1} & u_{n-2, n}-u_{n-2, n-1} u_{n-1, n} \\
& & & & & 1 & 0
\end{array}\right) \\
& \times\left(\begin{array}{ccccccc}
1 & u_{1,2} & \cdots & u_{1, n-3} & 0 & 0 & 0 \\
& 1 & \cdots & u_{2, n-3} & 0 & 0 & 0 \\
& & \ddots & \vdots & \vdots & \vdots & \vdots \\
& & & 1 & 0 & 0 & 0 \\
& & & & 1 & 0 & 0 \\
& & & & & 1 & u_{n-1, n} \\
& & & & & & 1
\end{array}\right) .
\end{aligned}
$$

A simple change of variable $u_{i, n}-u_{i, n-1} u_{n-1, n} \mapsto u_{i, n}$ with the fact that $f$ is automorphic give the desired result again by Proposition 2.2. Finally $a_{(0, \ldots, 0)}=0$ is a direct result of the last part of Proposition 2.2.

2.3. Incomplete Eisenstein Series and Spectral Decomposition. Eisenstein series induced form Maass forms play a central role in the spectral decomposition of the space $\mathfrak{L}\left(S L_{n}(\mathbb{Z}) \backslash X_{n}\right)$. But these 
Eisenstein series fail to be in $\mathfrak{L}\left(S L_{n}(\mathbb{Z}) \backslash X_{n}\right)$. So we need the theory of incomplete Eisenstein series. (They are referred as pseudo-Eisenstein in $[\mathrm{MW}]$ ). Incomplete Eisenstein series were used in the study of quantum ergodicity of $G L(2)$ Eisenstein series in [LS]. The analogous theory of incomplete Eisenstein series in more general settings can be found in $[\mathrm{L}, \mathrm{MW}, \mathrm{Ve}]$. Here we give a brief summary for this theory on $G L(n)$ explicitly. First we clarify the definition of Mellin transform.

Definition 2.5. For $\eta \in C_{0}^{\infty}\left(\left(\mathbb{R}^{+}\right)^{n}\right)$, we define the $n$-dimensional Mellin transform of $\eta$ to be

$$
\tilde{\eta}\left(s_{1}, \ldots, s_{n}\right)=\int_{0}^{\infty} \cdots \int_{0}^{\infty} \eta\left(y_{1}, \ldots, y_{n}\right) y_{1}^{-s_{1}} \cdots y_{n}^{-s_{n}} \frac{d y_{1} \cdots d y_{n}}{y_{1} \cdots y_{n}}
$$

Definition 2.6. For $\eta \in C_{0}^{\infty}\left(\left(\mathbb{R}^{+}\right)^{r-1}\right)$, we define the incomplete Eisenstein series $E_{\left(n_{1}, \ldots, n_{r}\right)}\left(z, \eta, \phi_{1}, \ldots, \phi_{r}\right)$ associated to $E_{\left(n_{1}, \ldots, n_{r}\right)}\left(z, s, \phi_{1}, \ldots, \phi_{r}\right)$ by

$E_{\left(n_{1}, \ldots, n_{r}\right)}\left(z, \eta, \phi_{1}, \ldots, \phi_{r}\right)=\frac{1}{(2 \pi i)^{r-1}} \int_{(2)} \cdots \int_{(2)} \tilde{\eta}\left(s_{1}, \ldots, s_{r-1}\right) E_{\left(n_{1}, \ldots, n_{r}\right)}\left(z, s, \phi_{1}, \ldots, \phi_{r}\right) d s_{1} \cdots d s_{r-1}$.

Note that the convergence of these integrals in Definition 2.6 are guaranteed by the rapid decay of $\tilde{\eta}$ in imaginary parts of the arguments.

Let $\mathcal{H}_{\text {cusp }}$ denote the space of $G L(n)$ cusp forms, $\mathcal{H}_{\left(n_{1}, \ldots, n_{r}\right)}$ denote the space spanned by incomplete Eisenstein series $E_{\left(n_{1}, \ldots, n_{r}\right)}\left(z, \eta, \phi_{1}, \ldots, \phi_{r}\right)$. The following theorem is a summary of the results presented in Section II of [MW] applied to the group $G L(n)$.

Theorem 2.2. We have the following spectral decomposition of automorphic forms on $G L(n)$ :

$$
\mathfrak{L}\left(S L_{n}(\mathbb{Z}) \backslash X_{n}\right)=\mathcal{H}_{\text {cusp }} \oplus \bigoplus_{\left(\left(n_{1}, \ldots, n_{r}\right)\right)} \mathcal{H}_{\left(n_{1}, \ldots, n_{r}\right)} .
$$

Note that $\mathcal{H}_{\left(n_{1}, \ldots, n_{r}\right)}=\mathcal{H}_{\left(n_{1}^{\prime}, \ldots, n_{r}^{\prime}\right)}$ if $\left(n_{1}, \ldots, n_{r}\right)$ and $\left(n_{1}^{\prime}, \ldots, n_{r}^{\prime}\right)$ are associate. So $\left(\left(n_{1}, \ldots, n_{r}\right)\right)$ means that the decomposition is over representatives of associate partitions. For convenience, we always choose the partition in non-increasing order.

From this decomposition, we will prove our main theorem by estimating the following integrals as $t \rightarrow \infty$ as mentioned in the introduction:

$$
\begin{aligned}
& \int_{S L_{n}(\mathbb{Z}) \backslash X_{n}} \phi(z)\left|E_{n-1,1}\left(z, \frac{1}{2}+i t, 1\right)\right|^{2} d^{*} z \\
& \int_{S L_{n}(\mathbb{Z}) \backslash X_{n}} E_{1, \ldots, 1}(z, \eta)\left|E_{n-1,1}\left(z, \frac{1}{2}+i t, 1\right)\right|^{2} d^{*} z \\
& \int_{S L_{n}(\mathbb{Z}) \backslash X_{n}} E_{n_{1}, \ldots, n_{r}}\left(z, \psi, u_{1}, \ldots, u_{r}\right)\left|E_{n-1,1}\left(z, \frac{1}{2}+i t, 1\right)\right|^{2} d^{*} z .
\end{aligned}
$$

where $\phi(z)$ is a $G L(n)$ cusp form. 


\section{Fourier Expansion of Degenerate Eisenstein Series}

3.1. Fourier Expansion of Degenerate Eisenstein Series. In this section, we present a complete calculation of the Fourier expansion of the degenerate Eisenstein series.

Theorem 3.1. The degenerate Eisenstein series for $G L(n)$ has only degenerate terms in its FourierWhittaker expansion for $n \geq 3$. More precisely,

$$
E_{(n-1,1)}(z, s, 1)=\sum_{m_{1} \in \mathbb{Z}} \hat{\phi}_{\left(m_{1}, 0, \ldots, 0\right)}(z)+\sum_{i=2}^{n-1} \sum_{\gamma_{i} \in P_{(i-1,1)}(\mathbb{Z}) \backslash S L_{i}(\mathbb{Z})} \sum_{m_{i}=1}^{\infty} \hat{\phi}_{\left(0, \ldots, 0, m_{i}, 0 \ldots, 0\right)}\left(\left(\begin{array}{ll}
\gamma_{i} & \\
& I_{n-i}
\end{array}\right) z\right) .
$$

where

$\hat{\phi}_{\left(m_{1}, \ldots, m_{n-1}\right)}(z, s):=\int_{0}^{1} \cdots \int_{0}^{1} E_{(n-1,1)}(u \cdot z, s, 1) e\left(-m_{1} u_{1,2}-m_{2} u_{2,3}-\cdots-m_{n-1} u_{n-1, n}\right) \prod_{1 \leq i<j \leq n} d u_{i, j}$

with $u \in U_{n}(\mathbb{R})$ and $I_{n-i}$ is the identity matrix of dimension $n-i$.

Furthermore, the coefficients are calculated to be

$$
\begin{gathered}
\hat{\phi}_{(0, \ldots, 0)}(z, s)=\sum_{k=0}^{n-1} \frac{2 \xi(n s-n+k+1)}{\xi(n s)}\left(y_{1} y_{2}^{2} \cdots y_{n-k-1}^{n-k-1}\right)^{(1-s)}\left(y_{n-k}^{k} y_{n-(k-1)}^{k-1} \cdots y_{n-1}\right)^{s} ; \\
\hat{\phi}_{\left(0, \ldots, 0, m_{k}, 0, \ldots, 0\right)}(z, s)= \\
\quad e\left(m_{k} x_{k, k+1}\right) \frac{2}{\xi(n s)}\left|m_{k}\right|^{\frac{n s}{2}-\frac{n-k}{2}} \sigma_{-n s+n-k}\left(\left|m_{k}\right|\right) K_{\frac{n s}{2}-\frac{n-k}{2}}\left(2 \pi\left|m_{k}\right| y_{n-k}\right) \\
\times\left(y_{1} y_{2}^{2} \cdots y_{n-k-1}^{n-k-1}\right)^{(1-s)}\left(y_{n-k}^{k} y_{n-(k-1)}^{k-1} \cdots y_{n-1}\right)^{s} y_{n-k}^{-\frac{n s}{2}+\frac{n-k}{2}} .
\end{gathered}
$$

We devote the reminding part of this section to proving the theorem. First rewrite the degenerate Eisenstein series in terms of Epstein Zeta function (see Section 10.7 of [Go] for a proof):

$$
\zeta(n s) E_{(n-1,1)}(z, s, 1)=\left(y_{1}^{n-1} y_{2}^{n-2} \cdots y_{n-1}\right)^{s} \sum_{\left(a_{1}, \ldots, a_{n}\right) \in \mathbb{Z}^{n} \backslash\{0\}}\left(b_{1}^{2}+\cdots+b_{n}^{2}\right)^{-n s / 2} .
$$

For

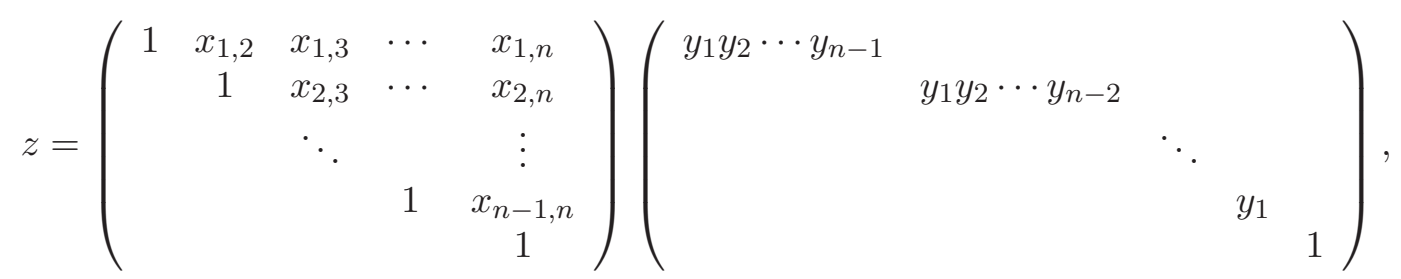

the $b_{i}$ 's are defined by

$$
\begin{aligned}
b_{1} & =a_{1} y_{1} \cdots y_{n-1} \\
b_{2} & =\left(a_{1} x_{1,2}+a_{2}\right) y_{1} \cdots y_{n-2} \\
& \vdots \\
b_{n} & =\left(a_{1} x_{1, n}+a_{2} x_{2, n}+\cdots+a_{n-1} x_{n-1, n}+a_{n}\right) .
\end{aligned}
$$

Let

$$
E_{(n-1,1)}^{*}(z, s):=\sum_{\left(a_{1}, \ldots, a_{n}\right) \in \mathbb{Z}^{n} \backslash\{0\}}\left(b_{1}^{2}+\cdots+b_{n}^{2}\right)^{-n s / 2} .
$$


First, we separate the terms with $a_{1}=0$ :

$$
\begin{aligned}
E_{(n-1,1)}^{*}(z, s) & =\sum_{\substack{\left(a_{2}, \ldots, a_{n}\right) \in \mathbb{Z}^{n-1} \backslash\{0\} \\
a_{1}=0}}\left(b_{2}^{2}+\cdots+b_{n}^{2}\right)^{-n s / 2}+\sum_{a_{1} \neq 0} \sum_{\left(a_{2}, \ldots, a_{n}\right) \in \mathbb{Z}^{n-1}}\left(b_{1}^{2}+\cdots+b_{n}^{2}\right)^{-n s / 2} \\
& =E_{(n-2,1)}^{*}\left(\pi_{n, n-1}(z), \frac{n}{n-1} s\right)+\mathcal{E}_{n}(z, s), \text { say. }
\end{aligned}
$$

Here $\pi_{n, n-k}: X_{n} \rightarrow X_{n-k}$ is the projection map:

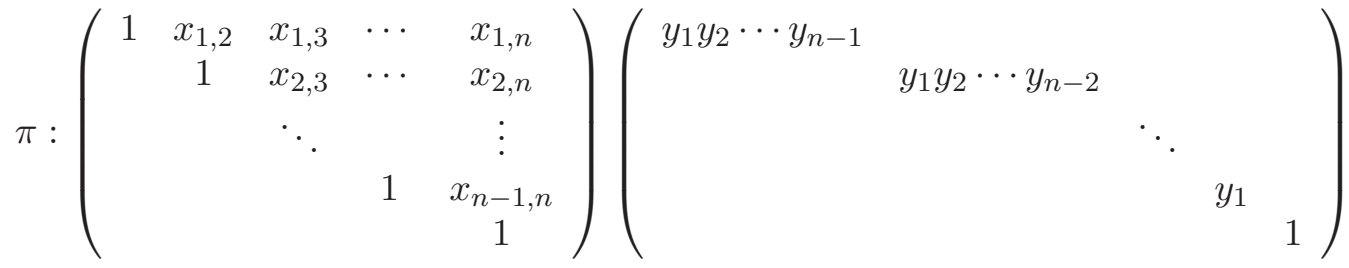

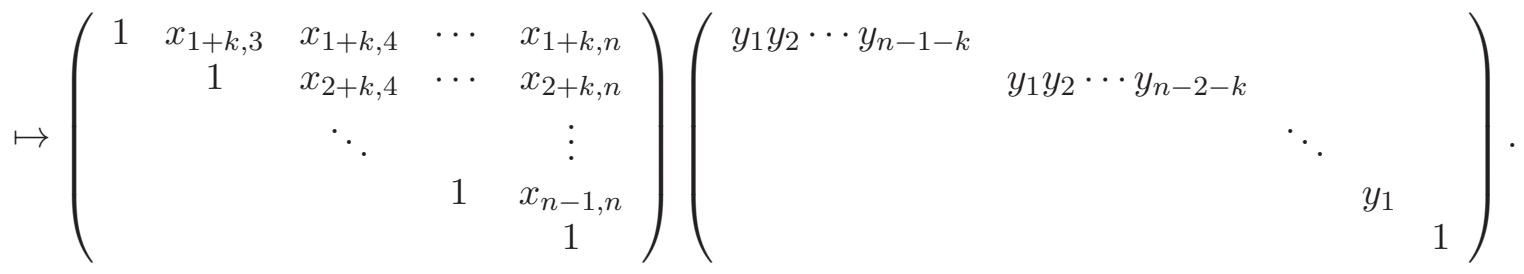

By Poisson summation,

$$
\mathcal{E}_{n}(z, s)=\sum_{a_{1} \neq 0} \sum_{\left(c_{2}, \ldots, c_{n}\right) \in \mathbb{Z}^{n-1}} \int_{-\infty}^{\infty} \cdots \int_{-\infty}^{\infty} \frac{e\left(-c_{2} a_{2}-\cdots-c_{n} a_{n}\right)}{\left(b_{1}^{2}+\cdots+b_{n}^{2}\right)^{n s / 2}} d a_{2} \cdots d a_{n} .
$$

Thus the Fourier-Whittaker coefficients of $E_{(n-1,1)}^{*}(z, s)$ are separated into two parts:

$$
\begin{aligned}
\frac{\zeta(n s)}{\left(y_{1}^{n-1} y_{2}^{n-2} \cdots y_{n-1}\right)^{s}} \hat{\phi}_{\left(m_{1}, \ldots, m_{n-1}\right)}(z, s)= & \int_{0}^{1} \cdots \int_{0}^{1}\left(E_{(n-2,1)}^{*}\left(\pi_{n, n-1}(u \cdot z), \frac{n}{n-1} s\right)+\mathcal{E}_{n}(u \cdot z, s)\right) \\
& \times e\left(-m_{1} u_{1,2}-m_{2} u_{2,3}-\cdots-m_{n-1} u_{n-1, n}\right) \prod_{1 \leq i<j \leq n} d u_{i, j} \\
= & \hat{\phi}_{\left(m_{1}, \ldots, m_{n-1}\right)}^{1}(z, s)+\hat{\phi}_{\left(m_{1}, \ldots, m_{n-1}\right)}^{2}(z, s),
\end{aligned}
$$

say. We treat these two parts in the following two lemmata.

Lemma 3.1. The first parts of the coefficients $\hat{\phi}_{\left(m_{1}, \ldots, m_{n-1}\right)}^{1}(z, s)$ have the following properties:

(1) $\hat{\phi}_{\left(m_{1}, \ldots, m_{n-1}\right)}^{1}(z, s)=0$ if $m_{1} \neq 0$;

(2) $\hat{\phi}_{\left(0, m_{2}, \ldots, m_{n-1}\right)}^{1}(z, s)=\frac{\zeta(n s)}{\left(y_{1}^{n-2} y_{2}^{n-3} \cdots y_{n-2}\right)^{\frac{n}{n-1} s}} \hat{\phi}_{\left(m_{2}, \ldots, m_{n-1}\right)}\left(\pi_{n, n-1}(z), \frac{n}{n-1} s\right)$.

Proof. By direct matrix multiplication, we find that

$$
\pi_{n, n-1}(u \cdot z)=\pi_{n, n-1}(u) \cdot \pi_{n, n-1}(z) .
$$

Thus the integral against the variable $u_{1,2}$ gives 0 unless $m_{1}=0$. This proves part (1). 
For part (2), again by equation 3.2 the integrals against the variables $u_{1, j}$ for $2 \leq j \leq n$ have no effect. So

$$
\begin{aligned}
& \int_{0}^{1} \cdots \int_{0}^{1} E_{(n-2,1)}^{*}\left(\pi_{n, n-1}(u \cdot z), \frac{n}{n-1} s\right) e\left(-m_{1} u_{1,2}-m_{2} u_{2,3}-\cdots-m_{n-1} u_{n-1, n}\right) \prod_{1 \leq i<j \leq n} d u_{i, j} \\
= & \int_{0}^{1} \cdots \int_{0}^{1} E_{(n-2,1)}^{*}\left(\pi_{n, n-1}(u) \cdot \pi_{n, n-1}(z), \frac{n}{n-1} s\right) e\left(-m_{2} u_{2,3}-\cdots-m_{n-1} u_{n-1, n}\right) \prod_{2 \leq i<j \leq n} d u_{i, j} \\
= & \frac{\zeta(n s)}{\left(y_{1}^{n-2} y_{2}^{n-3} \cdots y_{n-2}\right)^{\frac{n}{n-1}} s} \hat{\phi}_{\left(m_{2}, \ldots, m_{n-1}\right)}\left(\pi_{n, n-1}(z), \frac{n}{n-1} s\right) .
\end{aligned}
$$

Lemma 3.2. The term $\hat{\phi}_{\left(m_{1}, \ldots, m_{n-1}\right)}^{2}(z, s)$ in equation 3.1 can be nonzero only if $m_{2}=\cdots=m_{n-1}=0$. For $m_{1} \neq 0$

$$
\begin{aligned}
\hat{\phi}_{\left(m_{1}, 0 \ldots, 0\right)}^{2}(z, s)= & e\left(m_{1} x_{1,2}\right) \frac{2 \pi^{\frac{n s}{2}}}{\Gamma\left(\frac{n s}{2}\right)}\left|m_{1}\right|^{\frac{n s}{2}-\frac{n-1}{2}} \sigma_{-n s+n-1}\left(\left|m_{1}\right|\right)\left(y_{1}^{n-3} \cdots y_{n-3}\right)^{-1}\left(y_{1} \cdots y_{n-2}\right)^{-(n s-n+2)} \\
& \times y_{n-1}^{-\frac{n s}{2}+\frac{n-1}{2}} K_{\frac{n s}{2}-\frac{n-1}{2}}\left(2 \pi\left|m_{1}\right| y_{n-1}\right)
\end{aligned}
$$

for $m_{1}=0$

$$
\begin{aligned}
& \hat{\phi}_{(0, \ldots, 0)}^{2}(z, s) \\
= & \frac{2 \pi^{\frac{n-1}{2}} \zeta(n s-n+1) \Gamma\left(\frac{n s}{2}-\frac{n-1}{2}\right)}{\Gamma\left(\frac{n s}{2}\right)}\left(y_{1}^{n-2} \cdots y_{n-2}\right)^{-1}\left(y_{1} \cdots y_{n-1}\right)^{-n s+n-1} .
\end{aligned}
$$

Proof. Let $x^{\prime}$ denote the product of matrices $u \cdot x$. Define $b_{i}^{\prime}$ to be the same as $b_{i}$ with $x$ replaced by $x^{\prime}$. We now make the change of variable $b_{n}^{\prime} \mapsto a_{n}$ :

$$
\begin{aligned}
\hat{\phi}_{\left(m_{1}, \ldots, m_{n-1}\right)}^{2}(z, s)= & \int_{0}^{1} \cdots \int_{0}^{1} \sum_{a_{1} \neq 0} \sum_{\left(c_{2}, \ldots, c_{n}\right) \in \mathbb{Z}^{n-1}} \int_{-\infty}^{\infty} \cdots \int_{-\infty}^{\infty} \\
& \frac{e\left(-c_{2} a_{2}-\cdots-c_{n}\left(a_{n}-a_{1} x_{1, n}^{\prime}-\ldots-a_{n-1} x_{n-1, n}^{\prime}\right)\right)}{\left(b_{1}^{\prime 2}+\cdots+a_{n}^{2}\right)^{n s / 2}} d a_{2} \cdots d a_{n} \\
& \times e\left(-m_{1} u_{1,2}-m_{2} u_{2,3}-\cdots-m_{n-1} u_{n-1, n}\right) \prod_{1 \leq i<j \leq n} d u_{i, j} .
\end{aligned}
$$

We notice that the only entry with $u_{1, n}$ in the matrix $x^{\prime}$ is the $x_{1, n}^{\prime}$ entry. As $a_{1} \neq 0$, the integral against $u_{1, n}$ gives 0 unless $c_{n}=0$. After this simplification we have

$$
\begin{aligned}
\hat{\phi}_{\left(m_{1}, \ldots, m_{n-1}\right)}^{2}(z, s)= & \int_{0}^{1} \cdots \int_{0}^{1} \sum_{a_{1} \neq 0} \sum_{\left(c_{2}, \ldots, c_{n-1}\right) \in \mathbb{Z}^{n-2}} \int_{-\infty}^{\infty} \cdots \int_{-\infty}^{\infty} \frac{e\left(-c_{2} a_{2}-\cdots-c_{n-1} a_{n-1}\right)}{\left(b_{1}^{\prime 2}+\cdots+a_{n}^{2}\right)^{n s / 2}} d a_{2} \cdots d a_{n} \\
& \times e\left(-m_{1} u_{1,2}-m_{2} u_{2,3}-\cdots-m_{n-1} u_{n-1, n}\right) \frac{\prod_{1 \leq i<j \leq n} d u_{i, j}}{d u_{1, n}}
\end{aligned}
$$


Now make the change of variable $\frac{b_{n-1}^{\prime}}{y_{1}} \mapsto a_{n-1}$ :

$$
\begin{aligned}
\hat{\phi}_{\left(m_{1}, \ldots, m_{n-1}\right)}^{2}(z, s)= & \int_{0}^{1} \cdots \int_{0}^{1} \sum_{a_{1} \neq 0} \sum_{\left(c_{2}, \ldots, c_{n-1}\right) \in \mathbb{Z}^{n-2}} \int_{-\infty}^{\infty} \cdots \int_{-\infty}^{\infty} \\
& \times \frac{e\left(-c_{2} a_{2}-\cdots-c_{n-1}\left(a_{n-1}-a_{1} x^{\prime}{ }_{1, n-1}-\cdots-a_{n-2} x^{\prime}{ }_{n-2, n-1}\right)\right)}{\left(b_{1}^{\prime 2}+\cdots+a_{n-1}^{2} y_{1}^{2}+a_{n}^{2}\right)^{n s / 2}} d a_{2} \cdots d a_{n} \\
& \times e\left(-m_{1} u_{1,2}-m_{2} u_{2,3}-\cdots-m_{n-1} u_{n-1, n}\right) \frac{\prod_{1 \leq i<j \leq n} d u_{i, j}}{d u_{1, n}}
\end{aligned}
$$

The only term with $u_{1, n-1}$ is in the entry $x_{1, n-1}^{\prime}$. Integrating against $u_{1, n-1}$ with the fact that $a_{1} \neq 0$ shows $c_{n-1}=0$. Continuing in this fashion, we get

$$
\begin{aligned}
\hat{\phi}_{\left(m_{1}, \ldots, m_{n-1}\right)}^{2}(z, s)= & \int_{0}^{1} \cdots \int_{0}^{1} \sum_{a_{1} \neq 0} \sum_{c_{2} \in \mathbb{Z}} \int_{-\infty}^{\infty} \cdots \int_{-\infty}^{\infty} \frac{e\left(-c_{2}\left(a_{2}-a_{1} x_{1,2}-a_{1} u_{1,2}\right)\right)}{\left(a_{1}^{2} y_{1}^{2} \cdots y_{n-1}^{2}+\cdots+a_{n}^{2}\right)^{n s / 2}} d a_{2} \cdots d a_{n} \\
& \times e\left(-m_{1} u_{1,2}-m_{2} u_{2,3}-\cdots-m_{n-1} u_{n-1, n}\right) \frac{\prod_{1 \leq i<j \leq n} d u_{i, j}}{d u_{1, n} \cdots d u_{1,3}} .
\end{aligned}
$$

Clearly, this is zero unless $m_{2}=\cdots=m_{n-1}=0$.

To continue, we will use the following well-known identities:

$$
\begin{aligned}
\int_{-\infty}^{\infty}\left(A^{2} x^{2}+C\right)^{-\nu} d x & =\sqrt{\pi} \frac{\Gamma\left(\nu-\frac{1}{2}\right)}{\Gamma(\nu)}|A|^{-1} C^{-\nu+\frac{1}{2}} \\
\int_{-\infty}^{\infty}\left(A^{2} x^{2}+C^{2}\right)^{-\nu} e(-D x) d x & =\frac{2 \pi^{\nu}}{\Gamma(\nu)}|A|^{-\nu-\frac{1}{2}}|D|^{\nu-\frac{1}{2}}|C|^{\frac{1}{2}-\nu} K_{\nu-\frac{1}{2}}\left(\frac{2 \pi|C D|}{A}\right),
\end{aligned}
$$

where $A, C, D \in \mathbb{R}$. Continuing from 3.3, we have

$$
\hat{\phi}_{\left(m_{1}, 0 \ldots, 0\right)}^{2}(z, s)=\int_{0}^{1} \sum_{a_{1} \neq 0} \sum_{c_{2} \in \mathbb{Z}} \int_{-\infty}^{\infty} \cdots \int_{-\infty}^{\infty} \frac{e\left(-c_{2}\left(a_{2}-a_{1} x_{1,2}-a_{1} u_{1,2}\right)\right)}{\left(a_{1}^{2} y_{1}^{2} \cdots y_{n-1}^{2}+\cdots+a_{n}^{2}\right)^{n s / 2}} d a_{2} \cdots d a_{n} e\left(-m_{1} u_{1,2}\right) d u_{1,2} .
$$

For $m_{1}=0$,

$$
\begin{aligned}
\hat{\phi}_{(0,0 \ldots, 0)}^{2}(z, s) & =\int_{0}^{1} \sum_{a_{1} \neq 0} \sum_{c_{2} \in \mathbb{Z}} \int_{-\infty}^{\infty} \cdots \int_{-\infty}^{\infty} \frac{e\left(-c_{2}\left(a_{2}-a_{1} x_{1,2}-a_{1} u_{1,2}\right)\right)}{\left(a_{1}^{2} y_{1}^{2} \cdots y_{n-1}^{2}+\cdots+a_{n}^{2}\right)^{n s / 2}} d a_{2} \cdots d a_{n} d u_{1,2} \\
& =\sum_{a_{1} \neq 0} \int_{-\infty}^{\infty} \cdots \int_{-\infty}^{\infty} \frac{1}{\left(a_{1}^{2} y_{1}^{2} \cdots y_{n-1}^{2}+\cdots+a_{n}^{2}\right)^{n s / 2}} d a_{2} \cdots d a_{n} \\
& =\frac{2 \pi^{\frac{n-1}{2}} \zeta(n s-n+1) \Gamma\left(\frac{n s}{2}-\frac{n-1}{2}\right)}{\Gamma\left(\frac{n s}{2}\right)}\left(y_{1}^{n-2} \cdots y_{n-2}\right)^{-1}\left(y_{1} \cdots y_{n-1}\right)^{-n s+n-1}
\end{aligned}
$$

after repeatedly applying 3.4. For $m_{1} \neq 0$,

$$
\begin{aligned}
\hat{\phi}_{\left(m_{1}, 0 \ldots, 0\right)}^{2}(z, s)= & \int_{0}^{1} \sum_{a_{1} \neq 0} \sum_{c_{2} \in \mathbb{Z}} \int_{-\infty}^{\infty} \cdots \int_{-\infty}^{\infty} \frac{e\left(-c_{2}\left(a_{2}-a_{1} x_{1,2}-a_{1} u_{1,2}\right)\right)}{\left(a_{1}^{2} y_{1}^{2} \cdots y_{n-1}^{2}+\cdots+a_{n}^{2}\right)^{n s / 2}} d a_{2} \cdots d a_{n} e\left(-m_{1} u_{1,2}\right) d u_{1,2} \\
= & e\left(m_{1} x_{1,2}\right) \sum_{\substack{a_{1} \neq 0 c_{2} \in \mathbb{Z} \\
a_{1} c_{2}=m_{1}}} \int_{-\infty}^{\infty} \cdots \int_{-\infty}^{\infty} \frac{e\left(-c_{2} a_{2}\right)}{\left(a_{1}^{2} y_{1}^{2} \cdots y_{n-1}^{2}+\cdots+a_{n}^{2}\right)^{n s / 2}} d a_{2} \cdots d a_{n} \\
= & e\left(m_{1} x_{1,2}\right) \frac{2 \pi^{\frac{n s}{2}}}{\Gamma\left(\frac{n s}{2}\right)}\left|m_{1}\right|^{\frac{n s}{2}-\frac{n-1}{2}} \sigma_{-n s+n-1}\left(\left|m_{1}\right|\right)\left(y_{1}^{n-3} \cdots y_{n-3}\right)^{-1}\left(y_{1} \cdots y_{n-2}\right)^{-(n s-n+2)} \\
& \times y_{n-1}^{-\frac{n s}{2}+\frac{n-1}{2}} K_{\frac{n s}{2}-\frac{n-1}{2}}\left(2 \pi\left|m_{1}\right| y_{n-1}\right) .
\end{aligned}
$$


Finally the calculation of the Fourier expansion is complete after a simple induction on $n$ using equation 3.1 .

3.2. Constant Term Computation. In this section, we utilize the Fourier expansion computation to investigate the following two constant terms:

$$
\begin{gathered}
\int_{(\mathbb{Z} \backslash \mathbb{R})^{n-1}} E_{(n-1,1)}(z, s, 1) \prod_{h=1}^{n-1} d x_{h, n}, \\
\int_{(\mathbb{Z} \backslash \mathbb{R})^{n-1}}\left|E_{(n-1,1)}\left(z, \frac{1}{2}+i t, 1\right)\right|^{2} \prod_{h=1}^{n-1} d x_{h, n}
\end{gathered}
$$

to prepare for calculation in next section. First, we need a lemma.

Lemma 3.3. Let $\gamma_{k}=\left(\begin{array}{ccccc} & * & & \\ & & & & \\ \gamma_{k, 1} & \gamma_{k, 2} & \cdots & \gamma_{k, k-1} & \gamma_{k, k}\end{array}\right) \in S L_{k}(\mathbb{Z})$ and let

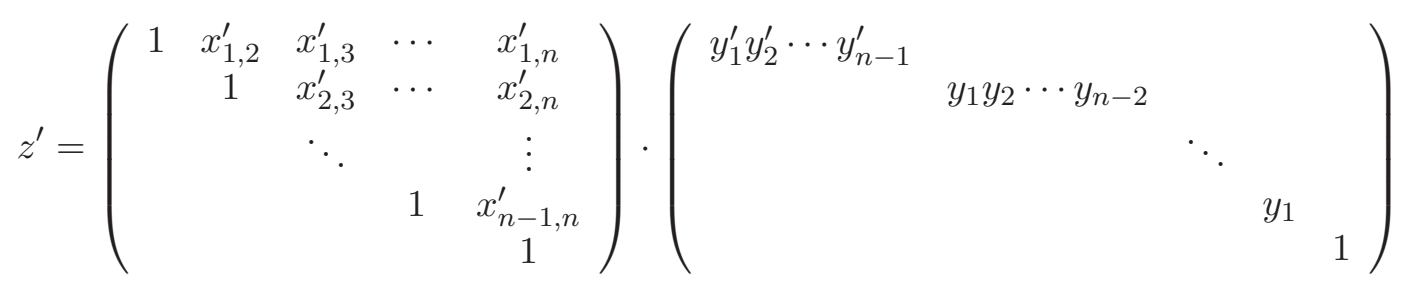

be the product $\left(\begin{array}{cc}\gamma_{k} & \\ & I_{n-k}\end{array}\right) \cdot z$ in Iwasawa form, then

$$
y_{j}^{\prime}=y_{j} \quad \text { for } 1 \leq j \leq n-k-1
$$

$$
y_{n-k}^{\prime k} y_{n-(k-1)}^{\prime k-1} \cdots y_{n-1}^{\prime}=y_{n-k}^{k} y_{n-(k-1)}^{k-1} \cdots y_{n-1}
$$

$$
y_{n-k}^{\prime}=y_{n-k}\left(b_{1}^{2}+\cdots+b_{k}^{2}\right)^{\frac{1}{2}}
$$

where

$$
\begin{aligned}
b_{1} & =\gamma_{k, 1} y_{n-k+1} \cdots y_{n-1} \\
b_{2} & =\left(\gamma_{k, 1} x_{1,2}+\gamma_{k, 2}\right) y_{n-k+1} \cdots y_{n-2} \\
& \vdots \\
b_{k-1} & =\left(\gamma_{k, 1} x_{1, k-1}+\gamma_{k, 2} x_{2, k-1}+\cdots \gamma_{k, k-2} x_{k-2, k-1}+\gamma_{k, k-1}\right) y_{n-k+1} \\
b_{k} & =\gamma_{k, 1} x_{1, k}+\gamma_{k, 2} x_{2, k}+\cdots \gamma_{k, k-1} x_{k-1, k}+\gamma_{k, k} .
\end{aligned}
$$


(4)

$$
x_{k, k+1}^{\prime}=\sum_{i=1}^{k} \gamma_{k, i} x_{i, k+1} .
$$

Proof. First we write

$$
x=\left(\begin{array}{cc}
X_{1} & V \\
& X_{2}
\end{array}\right) \quad y=\left(\begin{array}{cc}
Y_{1} y_{1} \cdots y_{n-k-1} & \\
& Y_{2}
\end{array}\right)=\left(\begin{array}{cc}
\tilde{Y}_{1} y_{1} \cdots y_{n-k} & \\
& Y_{2}
\end{array}\right),
$$

where $x \cdot y$ is the Iwasawa decomposition of $z$ and $X_{1}, Y_{1}$ are of dimension $k \times k, X_{2}, Y_{2}$ are of dimension $(n-k) \times(n-k)$. Matrix multiplication gives

$$
\left(\begin{array}{cc}
\gamma_{k} & \\
& I_{n-k}
\end{array}\right)\left(\begin{array}{cc}
X_{1} & V \\
& X_{2}
\end{array}\right)\left(\begin{array}{cc}
Y_{1} y_{1} \cdots y_{n-k-1} & \\
& Y_{2}
\end{array}\right)=\left(\begin{array}{cc}
\gamma_{k} \cdot X_{1} \cdot Y_{1} & \gamma_{k} \cdot V \\
& X_{2}
\end{array}\right)\left(\begin{array}{c}
I_{k} y_{1} \cdots y_{n-k-1} \\
Y_{2}
\end{array}\right) .
$$

By the Iwasawa decomposition, the matrix $\gamma_{i} \cdot X_{1} \cdot Y_{1}$ can be written as $X_{1}^{\prime} \cdot Y_{1}^{\prime} \cdot K_{1}^{\prime}$. So

$$
\left(\begin{array}{cc}
\gamma_{k} & \\
& I_{n-k}
\end{array}\right) z=\left(\begin{array}{cc}
X_{1}^{\prime} & \gamma_{k} \cdot V \\
& X_{2}
\end{array}\right)\left(\begin{array}{cc}
Y_{1}^{\prime} y_{1} \cdots y_{n-k-1} & \\
& Y_{2}
\end{array}\right)\left(\begin{array}{cc}
K^{\prime} & \\
& 1
\end{array}\right) \text {. }
$$

We have put $\left(\begin{array}{cc}\gamma_{k} & \\ & I_{n-k}\end{array}\right) z$ in Iwasawa form. Note that $\operatorname{det}\left(Y_{1}\right)=\operatorname{det}\left(Y_{1}^{\prime}\right)=y_{n-k}^{k} y_{n-(k-1)}^{k-1} \cdots y_{n-1}$ is invariant under the action of $\gamma_{k}$ as $\gamma_{k} \in S L_{k}(\mathbb{Z})$ has determinant 1 . At the same time, the variables $y_{1}, y_{2}, \ldots, y_{n-k-1}$ are unchanged.

The proof of the final part of this lemma is presented on P.309 of [Go].

\section{Proposition 3.1.}

$$
\begin{aligned}
& \int_{(\mathbb{Z} \backslash \mathbb{R})^{n-1}} E_{(n-1,1)}(z, s, 1) \prod_{h=1}^{n-1} d x_{h, n} \\
= & 2\left(y_{1}^{n-1} \cdots y_{n-1}\right)^{s}+\frac{\xi(n s-1)}{\xi(n s)}\left(y_{1}^{n-1} \cdots y_{n-1}\right)^{\frac{1-s}{n-1}} E_{(n-2,1)}\left(\mathfrak{m}_{n-1}(z), \frac{n s-1}{n-1}, 1\right) .
\end{aligned}
$$

Proof. By Theorem 3.1

$$
\begin{aligned}
& \int_{(\mathbb{Z} \backslash \mathbb{R})^{n-1}} E_{(n-1,1)}(z, s, 1) \prod_{h=1}^{n-1} d x_{h, n} \\
= & \int_{(\mathbb{Z} \backslash \mathbb{R})^{n-1}}\left(\sum_{m_{1} \in \mathbb{Z}} \hat{\phi}_{\left(m_{1}, 0, \ldots, 0\right)}(z)+\sum_{i=2}^{n-1} \sum_{\gamma_{i} \in P_{(i-1,1)}(\mathbb{Z}) \backslash S L_{i}(\mathbb{Z})} \sum_{m_{i}=1}^{\infty} \hat{\phi}_{\left(0, \ldots, 0, m_{i}, 0 \ldots, 0\right)}\left(\left(\begin{array}{cc}
\gamma_{i} & \\
& I_{n-i}
\end{array}\right) z\right)\right) \prod_{h=1}^{n-1} d x_{h, n}
\end{aligned}
$$

By the previous lemma, we see that only the term

$$
\sum_{\gamma_{i} \in P_{(i-1,1)}(\mathbb{Z}) \backslash S L_{i}(\mathbb{Z})} \sum_{m_{i}=1}^{\infty} \hat{\phi}_{\left(0, \ldots, 0, m_{i}, 0 \ldots, 0\right)}\left(\left(\begin{array}{cc}
\gamma_{i} & \\
& I_{n-i}
\end{array}\right) z\right)
$$

has the variables $x_{h, n}$ for $1 \leq h \leq n-1$ and these variables only appears in the exponential factor as $e\left(\sum_{j=1}^{n-1} \gamma_{n-1, j} x_{j, n}\right)$, where $\gamma_{a, b}$ are entries of the matrix $\gamma_{n-1}$. Thus

$$
\int_{(\mathbb{Z} \backslash \mathbb{R})^{n-1}} \sum_{\gamma_{i} \in P_{(i-1,1)}(\mathbb{Z}) \backslash S L_{i}(\mathbb{Z})} \sum_{m_{i}=1}^{\infty} \hat{\phi}_{\left(0, \ldots, 0, m_{i}, 0 \ldots, 0\right)}\left(\left(\begin{array}{cc}
\gamma_{i} & \\
& I_{n-i}
\end{array}\right) z\right) \prod_{h=1}^{n-1} d x_{h, n}=0 .
$$


So we have

$$
\begin{aligned}
& \int_{(\mathbb{Z} \backslash \mathbb{R})^{n-1}} E_{(n-1,1)}(z, s, 1) \prod_{h=1}^{n-1} d x_{h, n} \\
= & \sum_{m_{1} \in \mathbb{Z}} \hat{\phi}_{\left(m_{1}, 0, \ldots, 0\right)}(z)+\sum_{i=2}^{n-2} \sum_{\gamma_{i} \in P_{(i-1,1)}(\mathbb{Z}) \backslash S L_{i}(\mathbb{Z}) m_{m_{i}=1}}^{\infty} \hat{\phi}_{\left(0, \ldots, 0, m_{i}, 0 \ldots, 0\right)}\left(\left(\begin{array}{cc}
\gamma_{i} \\
I_{n-i}
\end{array}\right) z\right) \\
= & 2\left(y_{1}^{n-1} \cdots y_{n-1}\right)^{s}+\frac{\xi(n s-1)}{\xi(n s)}\left(y_{1}^{n-1} \cdots y_{n-1}\right)^{\frac{1-s}{n-1}} E_{(n-2,1)}\left(\mathfrak{m}_{n-1}(z), \frac{n s-1}{n-1}, 1\right)
\end{aligned}
$$

by comparing Fourier expansions.

\section{Proposition 3.2.}

$$
\begin{aligned}
& \int_{(\mathbb{Z} \backslash \mathbb{R})^{n-1}}\left|E_{(n-1,1)}(z, s, 1)\right|^{2} \prod_{h=1}^{n-1} d x_{h, n} \\
= & 4 \ell^{-2 n a}+\frac{|\xi(n s-1)|^{2}}{|\xi(n s)|^{2}}\left(y_{1}^{n-1} \cdots y_{n-1}\right)^{\frac{2-a}{n-1}}\left|E_{(n-2,1)}\left(\mathfrak{m}_{n-1}(z), \frac{n s-1}{n-1}, 1\right)\right|^{2} \\
& +2 \ell^{-\frac{n^{2} a-2 n a+n+i n^{2} b}{n-1}} \frac{\xi(n \bar{s}-1)}{\xi(n \bar{s})} E_{(n-2,1)}\left(\mathfrak{m}_{n-1}(z), \frac{n \bar{s}-1}{n-1}, 1\right) \\
& +2 \ell^{-\frac{n^{2} a-2 n a+n-i n^{2} b}{n-1}} \frac{\xi(n s-1)}{\xi(n s)} E_{(n-2,1)}\left(\mathfrak{m}_{n-1}(z), \frac{n s-1}{n-1}, 1\right) \\
& +\frac{8 \ell^{-2 n a}}{|\xi(s)|^{2}} \sum_{m_{n-1}=1}^{\infty} \gamma \in P_{(n-2,1)}(\mathbb{Z}) \backslash S L_{n-1}(\mathbb{Z}) \\
& \times\left. K_{\frac{n a-1}{2}+\frac{i n b}{2}}\left(2 \pi m_{n-1} \frac{\ell^{-\frac{n}{n-1}}}{\operatorname{det}\left(\mathfrak{m}_{n-1}(z)\right)^{\frac{1}{n-1}}}\right) K_{\frac{n a-1}{2}-\frac{i n b}{2}}\left(2 \pi m_{n-1} \frac{\ell^{-\frac{n}{n-1}}}{\operatorname{det}\left(\mathfrak{m}_{n-1}(z)\right)^{\frac{1}{n-1}}}\right)\right|_{\gamma}
\end{aligned}
$$

where $s=a+b i, \ell^{-n}=y_{1}^{n-1} \cdots y_{n-1}$ and $\operatorname{det}\left(\mathfrak{m}_{n-1}(z)\right)=y_{2}^{n-2} \cdots y_{n-1}$.

Proof. By the previous lemma we can open the square and find 


$$
\begin{aligned}
& \int_{(\mathbb{Z} \backslash \mathbb{R})^{n-1}}\left|E_{(n-1,1)}(z, s, 1)\right|^{2} \prod_{h=1}^{n-1} d x_{h, n} \\
& =\left|2\left(y_{1}^{n-1} \cdots y_{n-1}\right)^{s}+\frac{\xi(n s-1)}{\xi(n s)}\left(y_{1}^{n-1} \cdots y_{n-1}\right)^{\frac{1-s}{n-1}} E_{(n-2,1)}\left(\mathfrak{m}_{n-1}(z), \frac{n s-1}{n-1}, 1\right)\right|^{2} \\
& +\frac{8\left(y_{1}^{n-1} \cdots y_{n-1}\right)^{2 a}}{|\xi(s)|^{2}} \sum_{m_{n-1}=1}^{\infty} \sum_{\gamma \in P_{(n-2,1)}(\mathbb{Z}) \backslash S L_{n-1}(\mathbb{Z})} m_{n-1}^{n a-1} \sigma_{-n a+1-i n b}\left(m_{n-1}\right) \sigma_{-n a+1+i n b}\left(m_{n-1}\right) \\
& \times\left. K_{\frac{n a-1}{2}+\frac{i n b}{2}}\left(2 \pi m_{n-1} y_{1}\right) K_{\frac{n a-1}{2}-\frac{i n b}{2}}\left(2 \pi m_{n-1} y_{1}\right) y_{1}^{-n a+1}\right|_{\gamma} \\
& =4\left(y_{1}^{n-1} \cdots y_{n-1}\right)^{2 a}+\frac{|\xi(n s-1)|^{2}}{|\xi(n s)|^{2}}\left(y_{1}^{n-1} \cdots y_{n-1}\right)^{\frac{2-a}{n-1}}\left|E_{(n-2,1)}\left(\mathfrak{m}_{n-1}(z), \frac{n s-1}{n-1}, 1\right)\right|^{2} \\
& +2\left(y_{1}^{n-1} \cdots y_{n-1}\right)^{\frac{n a-2 a+1+i n b}{n-1}} \frac{\xi(n \bar{s}-1)}{\xi(n \bar{s})} E_{(n-2,1)}\left(\mathfrak{m}_{n-1}(z), \frac{n \bar{s}-1}{n-1}, 1\right) \\
& +2\left(y_{1}^{n-1} \cdots y_{n-1}\right)^{\frac{n a-2 a+1-i n b}{n-1}} \frac{\xi(n s-1)}{\xi(n s)} E_{(n-2,1)}\left(\mathfrak{m}_{n-1}(z), \frac{n s-1}{n-1}, 1\right) \\
& +\frac{8\left(y_{1}^{n-1} \cdots y_{n-1}\right)^{2 a}}{|\xi(s)|^{2}} \sum_{m_{n-1}=1}^{\infty} \sum_{\gamma \in P_{(n-2,1)}(\mathbb{Z}) \backslash S L_{n-1}(\mathbb{Z})} m_{n-1}^{n a-1} \sigma_{-n a+1-i n b}\left(m_{n-1}\right) \sigma_{-n a+1+i n b}\left(m_{n-1}\right) \\
& \times\left. K_{\frac{n a-1}{2}+\frac{i n b}{2}}\left(2 \pi m_{n-1} y_{1}\right) K_{\frac{n a-1}{2}-\frac{i n b}{2}}\left(2 \pi m_{n-1} y_{1}\right) y_{1}^{-n a+1}\right|_{\gamma}
\end{aligned}
$$

We get our desired result after the change of variable $\ell^{-n}=y_{1}^{n-1} \cdots y_{n-1}$ and $\operatorname{det}\left(\mathfrak{m}_{n-1}(z)\right)=y_{2}^{n-2} \cdots y_{n-1}$. 


\section{Cuspidal and Non-Minimal Eisenstein Contribution}

In this section we show that

(1)

$$
\int_{S L_{n}(\mathbb{Z}) \backslash X_{n}} \phi(z)\left|E_{n-1,1}\left(z, \frac{1}{2}+i t, 1\right)\right|^{2} d^{*} z=0,
$$

$(2)$

for $\phi(z)$ a cusp form on $G L(n)$ with $n \geq 3$.

$$
\int_{S L_{n}(\mathbb{Z}) \backslash X_{n}} E_{\left(n_{1}, \ldots, n_{r}\right)}\left(z, \psi, u_{1}, \ldots, u_{r}\right)\left|E_{n-1,1}\left(z, \frac{1}{2}+i t, 1\right)\right|^{2} d^{*} z=0
$$

(3)

if $n \geq 3$ and the partition $\left(n_{1}, \ldots, n_{r}\right)$ is not of type $(2,1, \ldots, 1)$.

$$
\int_{S L_{n}(\mathbb{Z}) \backslash X_{n}} E_{(2,1, \ldots, 1)}(z, \psi, \phi)\left|E_{n-1,1}\left(z, \frac{1}{2}+i t, 1\right)\right|^{2} d^{*} z=O_{\epsilon}\left(t^{-\frac{1}{2}+\epsilon}\right) .
$$

4.1. Some Basic Lemmata. Here we write down some basic lemmata that we will use repeatedly in the rest of the paper.

Lemma 4.1. (Stade's formula for $G L(2)$ )

The Mellin transform of product of $K$-Bessel functions can be evaluated (6.576.4 of [GR])

$$
\int_{0}^{\infty} K_{\mu}(y) K_{\nu}(y) y^{s} \frac{d y}{y}=2^{s-3} \frac{\Gamma\left(\frac{s+\mu+\nu}{2}\right) \Gamma\left(\frac{s+\mu-\nu}{2}\right) \Gamma\left(\frac{s-\mu+\nu}{2}\right) \Gamma\left(\frac{s-\mu-\nu}{2}\right)}{\Gamma(s)} .
$$

Lemma 4.2. We have the following beautiful formula given by Ramanujan [Ra].

$$
\sum_{n=1} \frac{\sigma_{a}(n) \sigma_{b}(n)}{n^{s}}=\frac{\zeta(s) \zeta(s-a) \zeta(s-b) \zeta(s-a-b)}{\zeta(2 s-a-b)}
$$

for $\operatorname{Re}(s)$ large enough.

Lemma 4.3. (Mellin inversion) (See 3.1.1 of $[\mathrm{PK}]$ )

The Mellin transform of a absolutely integrable function $f(x)$ on $(0, \infty)$ is defined by

$$
F(s)=\int_{0}^{\infty} x^{s-1} f(x) d x
$$

when the integral converges. If we suppose for small $\epsilon>0$

$$
f(x)=\left\{\begin{array}{lll}
O\left(x^{-a-\epsilon}\right) & \text { as } & x \rightarrow 0^{+} \\
O\left(x^{-b+\epsilon}\right) & \text { as } & x \rightarrow \infty
\end{array}\right.
$$

then for $a<c<b, f(x)$ can be recovered by Mellin inversion:

$$
f(x)=\frac{1}{2 \pi i} \int_{(c)} x^{-s} F(s) d s .
$$


4.2. Partition of the Form $n=n_{1}+\cdots+n_{r-1}+1$. We now fix a partition $n=n_{1}+\cdots+n_{r-1}+1 \neq 1+$ $\cdots+1$ in non-increasing order. Our goal this section is to reduce the calculation involving Eisenstein series associated to the parabolic subgroup $P_{n_{1}, \ldots, n_{r-1}, 1}$ to the calculation involving Eisenstein series associated to the parabolic subgroup $P_{n_{1}, \ldots, n_{r-1}}$. This argument can be repeated so that we are left with partitions of the form $n=n_{1}+\cdots+n_{r}$ with $n_{r} \geq 2$, which will be tackled in the next section.

First we have the following rearrangement of Eisenstein series

\section{Lemma 4.4.}

$$
\begin{aligned}
& E_{\left(n_{1}, \ldots, n_{r-1}, 1\right)}\left(z, s, \phi_{1}, \ldots, \phi_{r-1}, 1\right) \\
= & E_{(n-1,1)}\left(z, s^{\prime \prime}, E_{\left(n_{1}, \ldots, n_{r-1}\right)}\left(\mathfrak{m}_{n-1}(z), s^{\prime}, \phi_{1}, \ldots, \phi_{r-1}\right), 1\right)
\end{aligned}
$$

where $s^{\prime}=\left(s_{1}^{\prime}, \ldots, s_{r-1}^{\prime}\right) \in \mathbb{C}^{r-1}$ and $s^{\prime \prime} \in \mathbb{C}$ are given by

$$
s_{i}^{\prime}=s_{i}-\frac{n_{1} s_{1}+\cdots+n_{r-1} s_{r-1}}{n-1}, \quad s^{\prime \prime}=\frac{n_{1} s_{1}+\cdots+n_{r-1} s_{r-1}}{n-1} .
$$

Proof. By definition,

$$
\begin{aligned}
& E_{\left(n_{1}, \ldots, n_{r-1}, 1\right)}\left(z, s, \phi_{1}, \ldots, \phi_{r-1}, 1\right) \\
= & \sum_{\gamma \in P_{n_{1}, \ldots, n_{r-1}, 1}(\mathbb{Z}) \backslash S L_{n}(\mathbb{Z})} \prod_{i=1}^{r-1} \phi_{i}\left(\mathfrak{m}_{i}(\gamma z)\right) I_{s}\left(\gamma z, P_{n_{1}, \ldots, n_{r-1}, 1}\right) \\
= & \sum_{\beta \in P_{n_{1}, \ldots, n_{r-1}, 1}(\mathbb{Z}) \backslash P_{n-1,1}(\mathbb{Z})} \sum_{\alpha \in P_{n-1,1}(\mathbb{Z}) \backslash S L_{n}(\mathbb{Z})} \prod_{i=1}^{r-1} \phi_{i}\left(\mathfrak{m}_{i}(\beta \alpha z)\right) I_{s}\left(\beta \alpha z, P_{n_{1}, \ldots, n_{r-1}, 1}\right)
\end{aligned}
$$

Here we can write

$$
I_{s}\left(z, P_{n_{1}, \ldots, n_{r-1}, 1}\right)=I_{s^{\prime}}\left(\mathfrak{m}_{n-1}(z), P_{n_{1}, \ldots, n_{r-1}}\right)\left(y_{1}^{n-1} y_{2}^{n-2} \cdots y_{n-1}\right)^{s^{\prime \prime}} .
$$

where $s^{\prime}=\left(s_{1}^{\prime}, \ldots, s_{r-1}^{\prime}\right) \in \mathbb{C}^{r-1}$ and $s^{\prime \prime} \in \mathbb{C}$ are given by

$$
s_{i}^{\prime}=s_{i}-\frac{n_{1} s_{1}+\cdots+n_{r-1} s_{r-1}}{n-1}, \quad s^{\prime \prime}=\frac{n_{1} s_{1}+\cdots+n_{r-1} s_{r-1}}{n-1} .
$$

One easily can check that $n_{1} s_{1}^{\prime}+\cdots+n_{r-1} s_{r-1}^{\prime}=0$ so that $I_{s^{\prime}}\left(\mathfrak{m}_{n-1}(z), P_{n_{1}, \ldots, n_{r-1}}\right)$ is a well defined $I$-function with spectral parameter $s^{\prime}$. Thus we have

$$
\begin{aligned}
& E_{\left(n_{1}, \ldots, n_{r-1}, 1\right)}\left(z, s, \phi_{1}, \ldots, \phi_{r-1}, 1\right) \\
= & \left.\left.\sum_{\beta \in P_{n_{1}, \ldots, n_{r-1}, 1}(\mathbb{Z}) \backslash P_{n-1,1}(\mathbb{Z})} \sum_{\alpha \in P_{n-1,1}(\mathbb{Z}) \backslash S L_{n}(\mathbb{Z})} \prod_{i=1}^{r-1} \phi_{i}\left(\mathfrak{m}_{i}(z)\right) I_{s^{\prime}}\left(\mathfrak{m}_{n-1}(z), P_{n_{1}, \ldots, n_{r-1}}\right)\left(y_{1}^{n-1} y_{2}^{n-2} \cdots y_{n-1}\right)^{s^{\prime \prime}}\right|_{\beta}\right|_{\alpha} \\
= & \left.\sum_{\alpha \in P_{n-1,1}(\mathbb{Z}) \backslash S L_{n}(\mathbb{Z})} \sum_{\beta \in P_{n_{1}, \ldots, n_{r-1}, 1}(\mathbb{Z}) \backslash P_{n-1,1}(\mathbb{Z})}^{r-1} \prod_{i=1}^{r} \phi_{i}\left(\mathfrak{m}_{i}(\beta z)\right) I_{s^{\prime}}\left(\mathfrak{m}_{n-1}(\beta z), P_{n_{1}, \ldots, n_{r-1}}\right)\right)\left.\left(y_{1}^{n-1} y_{2}^{n-2} \cdots y_{n-1}\right)^{s^{\prime \prime}}\right|_{\alpha} \\
= & \left.\sum_{\alpha \in P_{n-1,1}(\mathbb{Z}) \backslash S L_{n}(\mathbb{Z})}\left(\sum_{\beta \in P_{n_{1}, \ldots, n_{r-1}}(\mathbb{Z}) \backslash S L_{n-1}(\mathbb{Z})} \prod_{i=1}^{r-1} \phi_{i}\left(\mathfrak{m}_{i}(\beta z)\right) I_{s^{\prime}}\left(\mathfrak{m}_{n-1}(\beta z), P_{n_{1}, \ldots, n_{r-1}}\right)\right)\left(y_{1}^{n-1} y_{2}^{n-2} \cdots y_{n-1}\right)^{s^{\prime \prime}}\right|_{\alpha} \\
= & \left.\sum_{\alpha \in P_{n-1,1}(\mathbb{Z}) \backslash S L_{n}(\mathbb{Z})} E_{\left(n_{1}, \ldots, n_{r-1}\right)}\left(\mathfrak{m}_{n-1}(z), s^{\prime}, \phi_{1}, \ldots, \phi_{r-1}\right)\left(y_{1}^{n-1} y_{2}^{n-2} \cdots y_{n-1}\right)^{s^{\prime \prime}}\right|_{\alpha} \\
= & E_{(n-1,1)}\left(z, s^{\prime \prime}, E_{\left(n_{1}, \ldots, n_{r-1}\right)}\left(\mathfrak{m}_{n-1}(z), s^{\prime}, \phi_{1}, \ldots, \phi_{r-1}\right), 1\right)
\end{aligned}
$$


as $\left(y_{1}^{n-1} y_{2}^{n-2} \cdots y_{n-1}\right)$ is invariant under the action of $\beta$ by Lemma 3.3.

Lemma 4.5. We have the decomposition of measurable space

$$
P_{n-1,1}(\mathbb{Z}) \backslash X_{n}=S L_{n-1}(\mathbb{Z}) \backslash X_{n-1} \times(\mathbb{R} / \mathbb{Z})^{n-1} \times \mathbb{R}^{+} .
$$

with

$$
d^{*} z=-\frac{1}{\xi(n)} d^{*} z^{\prime} \prod_{h=1}^{n-1} d x_{h, n} \ell^{n} \frac{d \ell}{\ell}
$$

where

$$
\ell=\left(\prod_{i=1}^{n-1} y_{i}^{n-i}\right)^{-\frac{1}{n}} \quad z^{\prime}=\mathfrak{m}_{n-1}(z)
$$

Proof. By matrix multiplication, we have

$$
\begin{aligned}
& z=X \cdot Y=\left(\begin{array}{ccccc}
1 & x_{1,2} & x_{1,3} & \cdots & x_{1, n} \\
& 1 & x_{2,3} & \cdots & x_{2, n} \\
& & \ddots & & \vdots \\
& & & 1 & x_{n-1, n} \\
& & & & 1
\end{array}\right)\left(\begin{array}{ccccc}
\ell y_{1} y_{2} \cdots y_{n-1} & & & & \\
& \ell y_{1} y_{2} \cdots y_{n-2} & & & \\
& & & & \\
& & & \ell y_{1} & \\
& & & & \ell
\end{array}\right) \\
& =\left(\begin{array}{ccccc}
1 & & & & x_{1, n} \\
& 1 & & & x_{2, n} \\
& & \ddots & & \vdots \\
& & & 1 & x_{n-1, n} \\
& & & & 1
\end{array}\right) \cdot\left(\begin{array}{cc}
z^{\prime} & \\
& 1
\end{array}\right) \cdot\left(\begin{array}{lll}
\ell^{-\frac{1}{n-1}} \cdot I_{n-1} & \\
& & \ell
\end{array}\right) \\
& =\left(\begin{array}{cc}
I_{n-1} & \bar{x}_{n} \\
& 1
\end{array}\right) \cdot\left(\begin{array}{cc}
z^{\prime} & \\
& 1
\end{array}\right) \cdot\left(\begin{array}{cc}
\ell^{-\frac{1}{n-1}} \cdot I_{n-1} & \\
&
\end{array}\right) \text {, }
\end{aligned}
$$

where

$$
z^{\prime}=\left(\begin{array}{ccccc}
1 & x_{1,2} & x_{1,3} & \cdots & x_{1, n-1} \\
& 1 & x_{2,3} & \cdots & x_{2, n-1} \\
& & \ddots & & \vdots \\
& & & 1 & x_{n-2, n-1} \\
& & & & 1
\end{array}\right)\left(\begin{array}{ccccc}
\ell^{\frac{n}{n-1}} y_{1} y_{2} \cdots y_{n-1} & & & & \\
& & \ell^{\frac{n}{n-1}} y_{1} y_{2} \cdots y_{n-2} & & \\
& & \ddots & \\
& & & & \ell^{\frac{n}{n-1}} y_{1}
\end{array}\right)=X^{\prime} \cdot Y^{\prime} .
$$

By direct computation, we have for $\gamma \in S L_{n-1}(\mathbb{Z})$

$$
\begin{aligned}
& \left(\begin{array}{ll}
\gamma & \\
& 1
\end{array}\right) \cdot z
\end{aligned}
$$

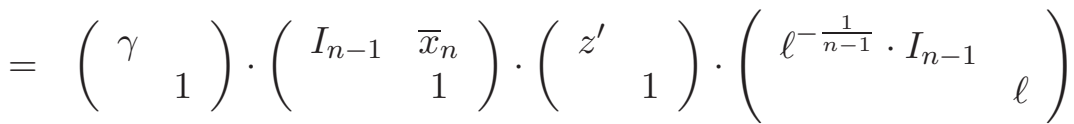

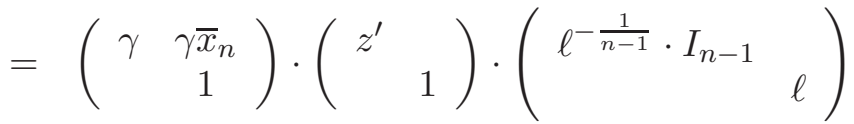

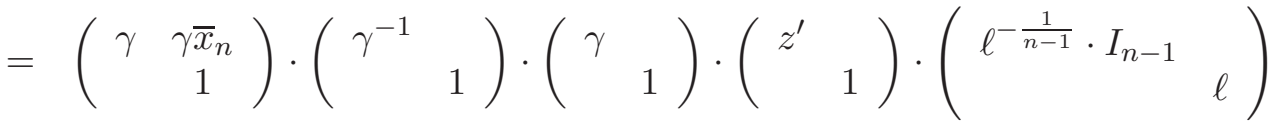

$$
\begin{aligned}
& =\left(\begin{array}{cc}
I_{n-1} & \gamma \bar{x}_{n} \\
& 1
\end{array}\right) \cdot\left(\begin{array}{cc}
\gamma z^{\prime} & \\
& 1
\end{array}\right) \cdot\left(\begin{array}{cc}
\ell^{-\frac{1}{n-1}} \cdot I_{n-1} & \\
&
\end{array}\right) \text {. }
\end{aligned}
$$


This gives the decomposition

$$
P_{n-1,1}(\mathbb{Z}) \backslash X_{n}=S L_{n-1}(\mathbb{Z}) \backslash X_{n-1} \times(\mathbb{R} / \mathbb{Z})^{n-1} \times \mathbb{R}^{+} .
$$

Let $F(z)$ be an automorphic function. We can unfold the following product:

$$
\begin{aligned}
& \int_{S L_{n} \backslash X_{n}} F(z) E_{n-1,1}(z, 1, s) d^{*} z \\
= & \int_{P_{n-1,1} \backslash X_{n}} F(z) \operatorname{det}(Y)^{s} d^{*} z \\
= & \int_{S L_{n-1}(\mathbb{Z}) \backslash X_{n-1}} \int_{0}^{\infty} \int_{(\mathbb{Z} / \mathbb{R})^{n-1}} F\left(\bar{x}_{n}, z^{\prime}, \ell\right) \ell^{-n s}\left(-\frac{n}{n-1}\right) d \bar{x}_{n} \ell^{n} \frac{d \ell}{\ell} d^{*} z^{\prime}
\end{aligned}
$$

Now define

$$
G\left(z^{\prime}\right):=\int_{0}^{\infty} \int_{(\mathbb{Z} / \mathbb{R})^{n-1}} F\left(\bar{x}_{n}, z^{\prime}, \ell\right) \ell^{-n s}\left(-\frac{n}{n-1}\right) d \bar{x}_{n} \ell^{n} \frac{d \ell}{\ell}
$$

We claim that $G\left(z^{\prime}\right)$ is automorphic.

$$
G\left(\gamma z^{\prime}\right)=\int_{0}^{\infty} \int_{(\mathbb{Z} / \mathbb{R})^{n-1}} F\left(\bar{x}_{n}, \gamma z^{\prime}, \ell\right) \ell^{-n s}\left(-\frac{n}{n-1}\right) d \bar{x}_{n} \ell^{n} \frac{d \ell}{\ell}
$$

With the fact that $F$ is automorphic we have

$$
\begin{aligned}
& F\left(\bar{x}_{n}, \gamma z^{\prime}, \ell\right) \\
& =F\left(\left(\begin{array}{cc}
I_{n-1} & \bar{x}_{n} \\
& 1
\end{array}\right) \cdot\left(\begin{array}{cc}
\gamma z^{\prime} & \\
& 1
\end{array}\right) \cdot\left(\begin{array}{ll}
\ell^{-\frac{1}{n-1}} \cdot I_{n-1} & \\
& \ell
\end{array}\right)\right) \\
& =F\left(\left(\begin{array}{cc}
\gamma & \\
& 1
\end{array}\right)\left(\begin{array}{cc}
I_{n-1} & \gamma^{-1} \bar{x}_{n} \\
& 1
\end{array}\right) \cdot\left(\begin{array}{cc}
z^{\prime} & \\
& 1
\end{array}\right) \cdot\left(\begin{array}{cc}
\ell^{-\frac{1}{n-1}} \cdot I_{n-1} & \\
&
\end{array}\right)\right) \\
& =F\left(\left(\begin{array}{cc}
I_{n-1} & \gamma^{-1} \bar{x}_{n} \\
& 1
\end{array}\right) \cdot\left(\begin{array}{ll}
z^{\prime} & \\
& 1
\end{array}\right) \cdot\left(\begin{array}{cc}
\ell^{-\frac{1}{n-1}} \cdot I_{n-1} & \\
&
\end{array}\right)\right) \text {. }
\end{aligned}
$$

Now since $\gamma \in S L_{n-1}(\mathbb{Z})$ and $F(z)$ is automorphic, the integral over $(\mathbb{Z} / \mathbb{R})^{n-1}$ is invariant under the action of $\gamma$ on $\bar{x}_{n}$. So

$$
\begin{aligned}
G\left(\gamma z^{\prime}\right) & =\int_{0}^{\infty} \int_{(\mathbb{Z} / \mathbb{R})^{n-1}} F\left(\bar{x}_{n}, \gamma z^{\prime}, \ell\right) \ell^{-n s}\left(-\frac{n}{n-1}\right) d \bar{x}_{n} \ell^{n} \frac{d \ell}{\ell} \\
& =\int_{0}^{\infty} \int_{(\mathbb{Z} / \mathbb{R})^{n-1}} F\left(\gamma^{-1} \bar{x}_{n}, z^{\prime}, \ell\right) \ell^{-n s}\left(-\frac{n}{n-1}\right) d \bar{x}_{n} \ell^{n} \frac{d \ell}{\ell} \\
& =\int_{0}^{\infty} \int_{(\mathbb{Z} / \mathbb{R})^{n-1}} F\left(\bar{x}_{n}, z^{\prime}, \ell\right) \ell^{-n s}\left(-\frac{n}{n-1}\right) d \bar{x}_{n} \ell^{n} \frac{d \ell}{\ell} \\
& =G\left(z^{\prime}\right) .
\end{aligned}
$$


Now let $\nu=a+b i$. By the definition of incomplete Eisenstein series and Lemma 4.5 we have

$$
\begin{aligned}
& \int_{S L_{n}(\mathbb{Z}) \backslash X_{n}} E_{\left(n_{1}, \ldots, n_{r-1}, 1\right)}\left(z, \eta, \phi_{1}, \ldots, \phi_{r-1}, 1\right)\left|E_{(n-1,1)}(z, \nu, 1)\right|^{2} d^{*} z \\
= & \int_{S L_{n}(\mathbb{Z}) \backslash X_{n}} \frac{1}{(2 \pi i)^{r-1}} \int_{(2)} \ldots \int_{(2)} \tilde{\eta}\left(s_{1}, \ldots, s_{r-1}\right) E_{\left(n_{1}, \ldots, n_{r-1}, 1\right)}\left(z, s, \phi_{1}, \ldots, \phi_{r-1}, 1\right) d s_{1} \cdots d s_{r-1} \\
& \times\left|E_{(n-1,1)}(z, \nu, 1)\right|^{2} d^{*} z \\
= & -\frac{1}{\xi(n)} \int_{S L_{n-1}(\mathbb{Z}) \backslash X_{n-1}} \int_{0}^{\infty} \int_{(\mathbb{Z} / \mathbb{R})^{n-1}} \frac{1}{(2 \pi i)^{r-1}} \int_{(2)} \cdots \int_{(2)} \tilde{\eta}\left(s_{1}, \ldots, s_{r-1}\right) \\
& E_{\left(n_{1}, \ldots, n_{r-1}\right)}\left(\mathfrak{m}_{n-1}(z), s^{\prime}, \phi_{1}, \ldots, \phi_{r-1}\right) \ell^{-n s^{\prime \prime}}\left|E_{(n-1,1)}(z, \nu, 1)\right|^{2} d s_{1} \cdots d s_{r-1} d^{*} z^{\prime} \prod_{h=1}^{n-1} d x_{h, n} \ell^{n} \frac{d \ell}{\ell}
\end{aligned}
$$

where $s^{\prime}=\left(s_{1}^{\prime}, \ldots, s_{r-1}^{\prime}\right) \in \mathbb{C}^{r-1}$ and $s^{\prime \prime} \in \mathbb{C}$ are given by

$$
s_{i}^{\prime}=s_{i}-\frac{n_{1} s_{1}+\cdots+n_{r-1} s_{r-1}}{n-1}, \quad s^{\prime \prime}=\frac{n_{1} s_{1}+\cdots+n_{r-1} s_{r-1}}{n-1} .
$$

as before.

Now by Proposition 3.2 we need to understand the following five integrals:

$$
\begin{aligned}
& \Xi_{1}=\frac{-1}{\xi(n)} \int_{S L_{n-1}(\mathbb{Z}) \backslash X_{n-1}} \int_{0}^{\infty} \frac{1}{(2 \pi i)^{r-1}} \int_{(2)} \cdots \int_{(2)} \tilde{\eta}(s) E_{\left(n_{1}, \ldots, n_{r-1}\right)}\left(z^{\prime}, s^{\prime}, \phi_{1}, \ldots, \phi_{r-1}\right) \ell^{-n s^{\prime \prime}} \\
& \times 4 \ell^{-2 n a} d s_{1} \cdots d s_{r-1} d^{*} z^{\prime} \ell^{n} \frac{d \ell}{\ell}, \\
& \Xi_{2}=\frac{-1}{\xi(n)} \int_{S L_{n-1}(\mathbb{Z}) \backslash X_{n-1}} \int_{0}^{\infty} \frac{1}{(2 \pi i)^{r-1}} \int_{(2)} \ldots \int_{(2)} \tilde{\eta}(s) E_{\left(n_{1}, \ldots, n_{r-1}\right)}\left(z^{\prime}, s^{\prime}, \phi_{1}, \ldots, \phi_{r-1}\right) \ell^{-n s^{\prime \prime}} \\
& \times \frac{|\xi(n \nu-1)|^{2}}{|\xi(n \nu)|^{2}} \ell^{-n \frac{2-a}{n-1}}\left|E_{(n-2,1)}\left(z^{\prime}, \frac{n \nu-1}{n-1}, 1\right)\right|^{2} d s_{1} \cdots d s_{r-1} d^{*} z^{\prime} \ell^{n} \frac{d \ell}{\ell}, \\
& \Xi_{3}=\frac{-1}{\xi(n)} \int_{S L_{n-1}(\mathbb{Z}) \backslash X_{n-1}} \int_{0}^{\infty} \frac{1}{(2 \pi i)^{r-1}} \int_{(2)} \cdots \int_{(2)} \tilde{\eta}(s) E_{\left(n_{1}, \ldots, n_{r-1}\right)}\left(z^{\prime}, s^{\prime}, \phi_{1}, \ldots, \phi_{r-1}\right) \ell^{-n s^{\prime \prime}} \\
& \times \ell^{-\frac{n^{2} a-2 n a+n+i n^{2} b}{n-1}} \frac{\xi(n \bar{\nu}-1)}{\xi(n \bar{\nu})} E_{(n-2,1)}\left(z^{\prime}, \frac{n \bar{\nu}-1}{n-1}, 1\right) d s_{1} \cdots d s_{r-1} d^{*} z^{\prime} \ell^{n} \frac{d \ell}{\ell}, \\
& \Xi_{3}^{\prime}=\frac{-1}{\xi(n)} \int_{S L_{n-1}(\mathbb{Z}) \backslash X_{n-1}} \int_{0}^{\infty} \frac{1}{(2 \pi i)^{r-1}} \int_{(2)} \cdots \int_{(2)} \tilde{\eta}(s) E_{\left(n_{1}, \ldots, n_{r-1}\right)}\left(z^{\prime}, s^{\prime}, \phi_{1}, \ldots, \phi_{r-1}\right) \ell^{-n s^{\prime \prime}} \\
& \times \ell^{-\frac{n^{2} a-2 n a+n-i n^{2} b}{n-1}} \frac{\xi(n \nu-1)}{\xi(n \nu)} E_{(n-2,1)}\left(z^{\prime}, \frac{n \nu-1}{n-1}, 1\right) d s_{1} \cdots d s_{r-1} d^{*} z^{\prime} \ell^{n} \frac{d \ell}{\ell},
\end{aligned}
$$




$$
\begin{aligned}
\Xi_{4}= & \frac{-1}{\xi(n)} \int_{S L_{n-1}(\mathbb{Z}) \backslash X_{n-1}} \int_{0}^{\infty} \frac{1}{(2 \pi i)^{r-1}} \int_{(2)} \cdots \int_{(2)} \tilde{\eta}(s) E_{\left(n_{1}, \ldots, n_{r-1}\right)}\left(z^{\prime}, s^{\prime}, \phi_{1}, \ldots, \phi_{r-1}\right) \ell^{-n s^{\prime \prime}} \\
& \times \frac{8 \ell^{-2 n a}}{|\xi(\nu)|^{2}} \sum_{m_{n-1}=1}^{\infty} \sum_{\gamma \in P_{(n-2,1)}(\mathbb{Z}) \backslash S L_{n-1}(\mathbb{Z})} m_{n-1}^{n a-1} \sigma_{-n a+1-i n b}\left(m_{n-1}\right) \sigma_{-n a+1+i n b}\left(m_{n-1}\right)\left(\frac{\ell^{-\frac{n}{n-1}}}{\operatorname{det}\left(z^{\prime}\right)^{\frac{1}{n-1}}}\right)^{-n a+1} \\
& \times\left. K_{\frac{n a-1}{2}+\frac{i n b}{2}}\left(2 \pi m_{n-1} \frac{\ell^{-\frac{n}{n-1}}}{\operatorname{det}\left(z^{\prime}\right)^{\frac{1}{n-1}}}\right) K_{\frac{n a-1}{2}-\frac{i n b}{2}}\left(2 \pi m_{n-1} \frac{\ell^{-\frac{n}{n-1}}}{\operatorname{det}\left(z^{\prime}\right)^{\frac{1}{n-1}}}\right)\right|_{\gamma} d s_{1} \cdots d s_{r-1} d^{*} z^{\prime} \ell^{n} \frac{d \ell}{\ell} .
\end{aligned}
$$

Lemma 4.6. For $\left(n_{1}, \ldots, n_{r-1}, 1\right) \neq(1, \ldots, 1)$, we have that $\Xi_{1}=0$.

Proof. Since $\left(n_{1}, \ldots, n_{r-1}, 1\right) \neq(1, \ldots, 1)$, the constant term of $E_{\left(n_{1}, \ldots, n_{r-1}\right)}\left(z^{\prime}, s^{\prime}, \phi_{1}, \ldots, \phi_{r-1}\right)$ along the minimal parabolic is 0 . So we are integrating an automorphic function without constant term over the entire quotient $S L_{n-1}(\mathbb{Z}) \backslash X_{n-1}$. We must have $\Xi_{1}=0$.

Lemma 4.7. For $\left(n_{1}, \ldots, n_{r-1}, 1\right) \neq(1, \ldots, 1)$, we have that $\Xi_{3}=\Xi_{3}^{\prime}=0$.

Proof. We can rearrange $\Xi_{3}$ and get

$$
\begin{aligned}
\Xi_{3}= & \frac{-1}{\xi(n)} \frac{\xi(n \bar{\nu}-1)}{\xi(n \bar{\nu})} \int_{S L_{n-1}(\mathbb{Z}) \backslash X_{n-1}} \int_{0}^{\infty} \frac{1}{(2 \pi i)^{r-1}} \int_{(2)} \cdots \int_{(2)} \tilde{\eta}(s) E_{\left(n_{1}, \ldots, n_{r-1}\right)}\left(z^{\prime}, s^{\prime}, \phi_{1}, \ldots, \phi_{r-1}\right) \ell^{-n s^{\prime \prime}} \\
& \times \ell^{-\frac{n^{2} a-2 n a+n+i n^{2} b}{n-1}} E_{(n-2,1)}\left(z^{\prime}, \frac{n \bar{\nu}-1}{n-1}, 1\right) d s_{1} \cdots d s_{r-1} d^{*} z^{\prime} \ell^{n} \frac{d \ell}{\ell}
\end{aligned}
$$
of

We can now apply the Mellin inversion theorem to eliminate the integral over $\ell$. Thus $\Xi_{3}$ has the shape

$$
\begin{aligned}
\Xi_{3}= & c \int_{\substack{S L_{n-1}(\mathbb{Z}) \backslash X_{n-1} \\
(2 \pi i)^{r-2}}} \frac{1}{(2)} \cdots \int_{(2)} f\left(s^{\prime}\right) E_{\left(n_{1}, \ldots, n_{r-1}\right)}\left(z^{\prime}, s^{\prime}, \phi_{1}, \ldots, \phi_{r-1}\right) \\
& \times E_{(n-2,1)}\left(z^{\prime}, \nu^{\prime}, 1\right) d s_{1}^{\prime} \cdots d s_{r-2}^{\prime} d^{*} z^{\prime}
\end{aligned}
$$

for some $f$ with rapid decay in the imaginary parts of the arguments. At this point, if $n_{r} \geq 2$, then unfolding with respect to $E_{(n-2,1)}\left(z^{\prime}, \nu^{\prime}, 1\right)$ and applying Proposition 2.2 reduce $\Xi_{3}$ to 0 . If $n_{r}=1$, we unfold with respect to $E_{\left(n_{1}, \ldots, n_{r-1}\right)}\left(z^{\prime}, s^{\prime}, \phi_{1}, \ldots, \phi_{r-1}\right)$ and apply 3.1 repeatedly until the last partition number is no longer 1 and apply Proposition 2.2.

Lemma 4.8. For $\left(n_{1}, \ldots, n_{r-1}, 1\right) \neq(1, \ldots, 1)$, we have that $\Xi_{4}=0$.

Proof.

$$
\begin{aligned}
\Xi_{4}= & \frac{-1}{\xi(n)} \int_{S L_{n-1}(\mathbb{Z}) \backslash X_{n-1}} \int_{0}^{\infty} \frac{1}{(2 \pi i)^{r-1}} \int_{(2)} \ldots \int_{(2)} \tilde{\eta}(s) E_{\left(n_{1}, \ldots, n_{r-1}\right)}\left(z^{\prime}, s^{\prime}, \phi_{1}, \ldots, \phi_{r-1}\right) \ell^{-n s^{\prime \prime}} \\
& \times \frac{8 \ell^{-2 n a}}{|\xi(\nu)|^{2}} \sum_{m_{n-1}=1}^{\infty} \sum_{\gamma \in P_{(n-2,1)}(\mathbb{Z}) \backslash S L_{n-1}(\mathbb{Z})} m_{n-1}^{n a-1} \sigma_{-n a+1-i n b}\left(m_{n-1}\right) \sigma_{-n a+1+i n b}\left(m_{n-1}\right)\left(\frac{\ell^{-\frac{n}{n-1}}}{\operatorname{det}\left(z^{\prime}\right)^{\frac{1}{n-1}}}\right)^{-n a+1} \\
& \times\left. K_{\frac{n a-1}{2}+\frac{i n b}{2}}\left(2 \pi m_{n-1} \frac{\ell^{-\frac{n}{n-1}}}{\operatorname{det}\left(z^{\prime}\right)^{\frac{1}{n-1}}}\right) K_{\frac{n a-1}{2}-\frac{i n b}{2}}\left(2 \pi m_{n-1} \frac{\ell^{-\frac{n}{n-1}}}{\operatorname{det}\left(z^{\prime}\right)^{\frac{1}{n-1}}}\right)\right|_{\gamma} d s_{1} \cdots d s_{r-1} d^{*} z^{\prime} \ell^{n} \frac{d \ell}{\ell}
\end{aligned}
$$

Recall that $\frac{\ell^{-\frac{n}{n-1}}}{\operatorname{det}\left(z^{\prime}\right)^{\frac{1}{n-1}}}=y_{1}$ and the effect of $\gamma$ on $y_{1}$ is given by part (3) of Lemma 3.3. We have $y_{1}^{\prime}=$ $y_{1}\left(b_{1}^{2}+\cdots+b_{n-1}^{2}\right)^{\frac{1}{2}}$ where $b_{i}^{\prime} s$ are defined in Lemma 3.3. We can then make the change of variable 
$m_{n-1} \frac{\ell^{-\frac{n}{n-1}}}{\operatorname{det}\left(z^{\prime}\right)^{\frac{1}{n-1}}}\left(b_{1}^{2}+\cdots+b_{n-1}^{2}\right)^{\frac{1}{2}} \mapsto u$. The sum of $\left(b_{1}^{2}+\cdots+b_{n-1}^{2}\right)^{\frac{1}{2}}$ over $P_{(n-2,1)}(\mathbb{Z}) \backslash S L_{n-1}(\mathbb{Z})$ is simply a degenerate Eisenstein series. The sum over $m_{n-1}$ of divisor functions is a ratio of Zeta functions by Lemma 4.2. This together with ratio of Gamma functions (formed after integrating over $u$ of the $K$-Bessel functions) will give completed Zeta functions. We can now apply the previous Lemma which shows that $\Xi_{4}=0$.

Proposition 4.1. For $\left(n_{1}, \ldots, n_{r-1}, 1\right) \neq(1, \ldots, 1)$, we have that

$$
\begin{aligned}
& \int_{S L_{n}(\mathbb{Z}) \backslash X_{n}} E_{\left(n_{1}, \ldots, n_{r-1}, 1\right)}\left(z, \eta, \phi_{1}, \ldots, \phi_{r-1}, 1\right)\left|E_{(n-1,1)}(z, \nu, 1)\right|^{2} d^{*} z \\
= & C \frac{|\xi(n \nu-1)|^{2}}{|\xi(n \nu)|^{2}} \int_{S L_{n-1}(\mathbb{Z}) \backslash X_{n-1}} \frac{1}{(2 \pi i)^{r-2}} \int_{(2)} \cdots \int_{(2)} \psi\left(s^{\prime}\right) E_{\left(n_{1}, \ldots, n_{r-1}\right)}\left(z^{\prime}, s^{\prime}, \phi_{1}, \ldots, \phi_{r-1}\right) \\
& \times\left|E_{(n-2,1)}\left(z^{\prime}, \frac{n \nu-1}{n-1}, 1\right)\right|^{2} d s_{1}^{\prime} \cdots d s_{r-2}^{\prime} d^{*} z^{\prime}
\end{aligned}
$$

for some absolute constant $C$.

Proof. From the previous three lemmas, we see that the only term left is $\Xi_{2}$. We have the following simplification for $\Xi_{2}$ :

$$
\begin{aligned}
\Xi_{2}= & \frac{-1}{\xi(n)} \int_{S L_{n-1}(\mathbb{Z}) \backslash X_{n-1}} \int_{0}^{\infty} \frac{1}{(2 \pi i)^{r-1}} \int_{(2)} \ldots \int_{(2)} \tilde{\eta}(s) E_{\left(n_{1}, \ldots, n_{r-1}\right)}\left(z^{\prime}, s^{\prime}, \phi_{1}, \ldots, \phi_{r-1}\right) \ell^{-n s^{\prime \prime}} \\
& \times \frac{|\xi(n \nu-1)|^{2}}{|\xi(n \nu)|^{2}} \ell^{-n \frac{2-a}{n-1}}\left|E_{(n-2,1)}\left(z^{\prime}, \frac{n \nu-1}{n-1}, 1\right)\right|^{2} d s_{1} \cdots d s_{r-1} d^{*} z^{\prime} \ell^{n} \frac{d \ell}{\ell} \\
= & -\frac{n^{2}-n}{n_{r-1}} \frac{|\xi(n \nu-1)|^{2}}{\xi(n)|\xi(n \nu)|^{2}} \int_{S L_{n-1}(\mathbb{Z}) \backslash X_{n-1}} \frac{1}{(2 \pi i)^{r-2}} \int_{(2)} \ldots \int_{(2)} \psi\left(s^{\prime}\right) E_{\left(n_{1}, \ldots, n_{r-1}\right)}\left(z^{\prime}, s^{\prime}, \phi_{1}, \ldots, \phi_{r-1}\right) \\
& \times\left|E_{(n-2,1)}\left(z^{\prime}, \frac{n \nu-1}{n-1}, 1\right)\right|^{2} d s_{1}^{\prime} \cdots d s_{r-2}^{\prime} d^{*} z^{\prime},
\end{aligned}
$$

where the factor $\frac{n^{2}-n}{n_{r-1}}$ comes from the Jacobian of the change of variables and

$$
\psi\left(s^{\prime}\right):=\tilde{\eta}\left(s_{1}^{\prime}+c, \ldots, s_{r-2}^{\prime}+c,-\left(s_{1}^{\prime}+\ldots+s_{r-2}^{\prime}+c\right)\right)
$$

where $c=\frac{2-a}{n-1}-1$.

This proposition can be used until we reach the following:

(1)

$$
\int_{S L_{2}(\mathbb{Z}) \backslash \mathbb{H}} \phi(z)|E(z, s)|^{2} d^{*} z
$$

$$
\int_{S L_{n}(\mathbb{Z}) \backslash X_{n}} \phi(z)\left|E_{n-1,1}(z, s, 1)\right|^{2} d^{*} z
$$

where $\phi$ is a cusp form on $G L(n)$ for $n \geq 3$.

$$
\int_{S L_{n}(\mathbb{Z}) \backslash X_{n}} E_{\left(n_{1}, \ldots, n_{r}\right)}\left(z, \eta, \phi_{1}, \ldots, \phi_{r}\right)\left|E_{(n-1,1)}(z, \nu, 1)\right|^{2} d^{*} z
$$


for $n=n_{1}+\ldots+n_{r}$ in non-increasing order with $n_{r} \geq 2$.

We will consider these three separately in the next three sections.

4.3. A $G L(2)$ Calculation. In this section, our goal is to show the following estimate.

\section{Theorem 4.1.}

$$
\int_{S L_{n}(\mathbb{Z}) \backslash X_{n}} E_{(2,1, \ldots, 1)}(z, \eta, \phi)\left|E_{n-1,1}\left(z, \frac{1}{2}+i t, 1\right)\right|^{2} d^{*} z \ll t^{-\frac{1}{2}+\epsilon} .
$$

Remark 4.1. We get this rate by only utilizing the convexity bounds for the Zeta function and L-functions associated to Maass forms.

Proof. First we apply Proposition $4.1(n-2)$ times and get

$$
\begin{aligned}
& \int_{S L_{n}(\mathbb{Z}) \backslash X_{n}} E_{(2,1, \ldots, 1)}(z, \eta, \phi)\left|E_{n-1,1}\left(z, \frac{1}{2}+i t, 1\right)\right|^{2} d^{*} z \\
\ll & \frac{\left|\xi\left(2-\frac{n}{2}+i n t\right)\right|^{2}}{\left|\xi\left(\frac{n}{2}+i n t\right)\right|^{2}} \int_{S L_{2}(\mathbb{Z}) \backslash X_{2}} \phi(z)\left|E\left(z, 1-\frac{n}{4}+i n t\right)\right|^{2} d^{*} z
\end{aligned}
$$

We follow the proof of Proposition 2.1 of [LS] closely. Consider

$$
\begin{aligned}
I_{\phi}(s) & :=\int_{S L_{2}(\mathbb{Z}) \backslash X_{2}} \phi(z) E\left(z, 1-\frac{n}{4}+i n t\right) E(z, s) d^{*} z \\
& =\int_{0}^{\infty} \int_{0}^{1} \phi(z) E\left(z, 1-\frac{n}{4}+i n t\right) y^{s} \frac{d x d y}{y^{2}}
\end{aligned}
$$

we will subsitute $s=1-\frac{n}{4}-i n t$ later in the proof. For odd forms this integral is 0 , so we only consider even forms. Fourier expansions of even forms $\phi(z)$ and $E(z, s)$ are given by:

$$
\begin{gathered}
\phi(z)=y^{\frac{1}{2}} \sum_{n=1}^{\infty} \rho_{\phi}(1) \lambda_{\phi}(n) K_{i t_{\phi}}(2 \pi n y) \cos (2 \pi n x), \\
E(z, s)=y^{s}+\frac{\xi(2 s-1)}{\xi(2 s)} y^{1-s}+\frac{2 y^{\frac{1}{2}}}{\xi(2 s)} \sum_{n=1}^{\infty} n^{s-\frac{1}{2}} \sigma_{1-2 s}(n) K_{s-\frac{1}{2}}(2 \pi n y) \cos (2 \pi n x) .
\end{gathered}
$$

Since $\phi$ is fixed, we can ignore the normalization constant $\rho_{\phi}(1)$. The spectral parameter $\lambda_{\phi}$ is related to $t_{\phi}$ by $\lambda_{\phi}=\frac{1}{4}+t_{\phi}^{2}$. The $L$-function associated to $u_{\phi}$ is defined by

$$
L(\phi, s):=\sum_{n=1}^{\infty} \frac{\lambda_{\phi}(n)}{n^{s}}=\prod_{p}\left(1-\lambda_{\phi}(p) p^{-s}+p^{-2 s}\right)^{-1} .
$$


So

$$
\begin{aligned}
I_{\phi}(s)= & \int_{0}^{\infty} \int_{0}^{1}\left(y^{\frac{1}{2}} \sum_{n=1}^{\infty} \lambda_{\phi}(n) K_{i t_{\phi}}(2 \pi n y) \cos (2 \pi n x)\right)\left(y^{1-\frac{n}{4}+i n t}+\frac{\xi\left(1-\frac{n}{2}+2 i n t\right)}{\xi\left(2-\frac{n}{2}+2 i n t\right)} y^{\frac{n}{4}-i n t}\right. \\
& \left.+\frac{2 y^{\frac{1}{2}}}{\xi\left(2-\frac{n}{2}+2 i n t\right)} \sum_{n^{\prime}=1}^{\infty} n^{\prime \frac{1}{2}-\frac{n}{4}+i n t} \sigma_{\frac{n}{2}-1-2 i n t}\left(n^{\prime}\right) K_{\frac{1}{2}-\frac{n}{4}+i n t}\left(2 \pi n^{\prime} y\right) \cos \left(2 \pi n^{\prime} x\right)\right) y^{s} \frac{d x d y}{y^{2}} \\
= & \frac{1}{\xi\left(2-\frac{n}{2}+2 i n t\right)} \int_{0}^{\infty} \sum_{n=1}^{\infty} \lambda_{\phi}(n) K_{i t_{\phi}}(2 \pi n y) n^{\frac{1}{2}-\frac{n}{4}+i n t} \sigma_{\frac{n}{2}-1-2 i n t}(n) K_{\frac{1}{2}-\frac{n}{4}+i n t}(2 \pi n y) y^{s} \frac{d y}{y} \\
= & \frac{1}{\xi\left(2-\frac{n}{2}+2 i n t\right)}\left(\sum_{n=1}^{\infty} \frac{\lambda_{\phi}(n) n^{\frac{1}{2}-\frac{n}{4}+i n t} \sigma_{\frac{n}{2}-1-2 i n t}(n)}{n^{s}}\right) \int_{0}^{\infty} K_{i t_{\phi}}(2 \pi y) K_{\frac{1}{2}-\frac{n}{4}+i n t}(2 \pi y) y^{s} \frac{d y}{y} .
\end{aligned}
$$

Following equation (15) of [LS], it can be shown that

$$
\sum_{n=1}^{\infty} \frac{\lambda_{\phi}(n) n^{\frac{1}{2}-\frac{n}{4}+i n t} \sigma_{\frac{n}{2}-1-2 i n t}(n)}{n^{s}}=\frac{L\left(\phi, s-\frac{1}{2}+\frac{n}{4}-i n t\right) L\left(\phi, s+\frac{1}{2}-\frac{n}{4}+i n t\right)}{\zeta(2 s)} .
$$

The Mellin transform of product of $K$-Bessel functions can be evaluated using 4.1, we have

$$
\begin{aligned}
& \int_{0}^{\infty} K_{i t_{\phi}}(2 \pi y) K_{\frac{1}{2}-\frac{n}{4}+i n t}(2 \pi y) y^{s} \frac{d y}{y} \\
= & \frac{2^{-3} \pi^{-s}}{\Gamma(s)} \Gamma\left(\frac{s+i t_{\phi}+\frac{1}{2}-\frac{n}{4}+i n t}{2}\right) \Gamma\left(\frac{s-i t_{\phi}+\frac{1}{2}-\frac{n}{4}+i n t}{2}\right) \Gamma\left(\frac{s+i t_{\phi}-\frac{1}{2}+\frac{n}{4}-i n t}{2}\right) \\
& \times \Gamma\left(\frac{s-i t_{\phi}-\frac{1}{2}+\frac{n}{4}-i n t}{2}\right) .
\end{aligned}
$$

Now let $s=1-\frac{n}{4}-i n t$, together we can estimate the following product by using Stirling's formula and the bound $L\left(u_{j}, \frac{1}{2}+i t\right) \ll|t|^{\frac{1}{2}+\epsilon}$ :

$$
\begin{aligned}
& \frac{\left|\xi\left(2-\frac{n}{2}+i n t\right)\right|^{2}}{\left|\xi\left(\frac{n}{2}+i n t\right)\right|^{2}} \frac{L\left(\phi, \frac{1}{2}-2 i n t\right) L\left(\phi, \frac{3-n}{2}\right)}{\xi\left(2-\frac{n}{2}+2 i n t\right) \xi\left(2-\frac{n}{2}-2 i n t\right)} \Gamma\left(\frac{\frac{3-n}{2}+i t_{\phi}}{2}\right) \Gamma\left(\frac{\frac{3-n}{2}-i t_{\phi}}{2}\right) \\
& \times \Gamma\left(\frac{\frac{1}{2}-2 i n t+i t_{\phi}}{2}\right) \Gamma\left(\frac{\frac{1}{2}-2 i n t-i t_{\phi}}{2}\right) \\
\ll_{\phi, \epsilon} & t^{-1} t^{\frac{1}{2}+\epsilon}=t^{-\frac{1}{2}+\epsilon} .
\end{aligned}
$$

\subsection{Cuspidal Contribution.}

\section{Proposition 4.2.}

$$
\int_{S L_{n}(\mathbb{Z}) \backslash X_{n}} \phi(z) E_{n-1,1}(z, s, 1) E_{n-1,1}\left(z, s^{\prime}, 1\right) d^{*} z=0 .
$$

for $n \geq 3$ and $\phi$ a Maass cusp form on $G L(n)$. 
Proof. Let $\phi(z)$ be a cusp form with Fourier-Whittaker expansion

$\phi(z)=\left.\sum_{\gamma \in U_{n-1}(\mathbb{Z}) \backslash S L_{n-1}(\mathbb{Z})} \sum_{m_{1}=1}^{\infty} \cdots \sum_{m_{n-2}=1}^{\infty} \sum_{m_{n-1} \neq 0} \frac{A\left(m_{1}, \ldots, m_{n-1}\right)}{\prod_{k=1}^{n-1}\left|m_{k}\right|^{k(n-k) / 2}} W_{\mathrm{Jac}}(M y, \nu) e\left(m_{1} x_{1}+\cdots+m_{n-1} x_{n-1}\right)\right|_{\gamma}$

where

$$
M=\left(\begin{array}{cccc}
m_{1} \cdots\left|m_{n}\right| & & & \\
& \ddots & & \\
& & m_{1} & \\
& & & 1
\end{array}\right) .
$$

Note we define the slash operator to be such that

$$
\left.f(z)\right|_{\gamma}=f\left(\left(\begin{array}{cc}
\gamma & \\
& 1
\end{array}\right) \cdot z\right) .
$$

By the Rankin-Selberg convolution on $G L(n)$,

$$
\begin{aligned}
& \int_{S L_{n}(\mathbb{Z}) \backslash X_{n}} \phi(z) E_{n}\left(z, s^{\prime}, 1\right) E_{n}(z, s, 1) d^{*} z \\
= & \int_{-\infty}^{\infty} \cdots \int_{-\infty}^{\infty} \int_{0}^{1} \cdots \int_{0}^{1} \sum_{m_{1}=1}^{\infty} \cdots \sum_{m_{n-2}=1}^{\infty} \sum_{m_{n-1} \neq 0} \frac{A\left(m_{1}, \ldots, m_{n-1}\right)}{\prod_{k=1}^{n-1}\left|m_{k}\right| k(n-k) / 2} W_{\mathrm{Jac}}(M y, \nu) e\left(m_{1} x_{1}+\cdots+m_{n-1} x_{n-1}\right) \\
& \times E_{n}\left(z, s^{\prime}, 1\right) \operatorname{det}(y)^{s} \prod_{1 \leq i<j \leq n-1} d x_{i, j} \prod_{k=1}^{n-1} \frac{d y_{k}}{y_{k}^{k(n-k)+1}} .
\end{aligned}
$$

To utilize the Fourier expansion

$$
E_{n}(z, s, 1)=\sum_{m_{1} \in \mathbb{Z}} \hat{\phi}_{\left(m_{1}, 0, \ldots, 0\right)}(z)+\sum_{i=2}^{n-1} \sum_{\gamma_{i} \in P_{i}(\mathbb{Z}) \backslash S L_{i}(\mathbb{Z})} \sum_{m_{i}=1}^{\infty} \hat{\phi}_{\left(0, \ldots, 0, m_{i}, 0 \ldots, 0\right)}\left(\left(\begin{array}{cc}
\gamma_{i} & \\
& I_{n-i}
\end{array}\right) z\right),
$$

we must understand the effect of $\left(\begin{array}{cc}\gamma_{i} & \\ & I_{n-i}\end{array}\right)$ on $x_{i, i+1}$. First we write

$$
x=\left(\begin{array}{cc}
X_{1} & V \\
& X_{2}
\end{array}\right) \quad y=\left(\begin{array}{cc}
Y_{1} y_{1} \cdots y_{n-i-1} & \\
& Y_{2}
\end{array}\right),
$$

where $x \cdot y$ is the Iwasawa decomposition of $z$ and $X_{1}, Y_{1}$ are of dimension $i \times i, X_{2}, Y_{2}$ are of dimension $(n-i) \times(n-i)$. Matrix multiplication gives

$$
\left(\begin{array}{cc}
\gamma_{i} & \\
& I_{n-i}
\end{array}\right)\left(\begin{array}{cc}
X_{1} & V \\
& X_{2}
\end{array}\right)\left(\begin{array}{cc}
Y_{1} y_{1} \cdots y_{n-i-1} & \\
& Y_{2}
\end{array}\right)=\left(\begin{array}{cc}
\gamma_{i} \cdot X_{1} \cdot Y_{1} & \gamma_{i} \cdot V \\
& X_{2}
\end{array}\right)\left(\begin{array}{c}
I_{i} y_{1} \cdots y_{n-i-1} \\
Y_{2}
\end{array}\right) .
$$

By the Iwasawa decomposition, the matrix $\gamma_{i} \cdot X_{1} \cdot Y_{1}$ can be written as $X_{1}^{\prime} \cdot Y_{1}^{\prime} \cdot K_{1}^{\prime}$. So

$$
\left(\begin{array}{cc}
\gamma_{i} & \\
& I_{n-i}
\end{array}\right) z=\left(\begin{array}{cc}
X_{1}^{\prime} & \gamma_{i} \cdot V \\
& X_{2}
\end{array}\right)\left(\begin{array}{cc}
Y_{1}^{\prime} y_{1} \cdots y_{n-i-1} & \\
& Y_{2}
\end{array}\right)\left(\begin{array}{cc}
K^{\prime} & \\
& 1
\end{array}\right)
$$

We have put $\left(\begin{array}{cc}\gamma_{i} & \\ & I_{n-i}\end{array}\right) z$ in Iwasawa form. In particular the $(i, i+1)$-entry becomes

$$
\sum_{\ell=1}^{i} \gamma_{i, \ell} x_{\ell, i+1}
$$

which appears in the bottom left corner of $\gamma_{i} \cdot V$. 
It is important to notice that $X_{1}^{\prime}$ and $Y_{1}^{\prime}$ are completed determined by $X_{1}, Y_{1}$ and $\gamma_{i}$. They do not have any relations to the matrix $V$. Thus the exponential factor $e\left(m_{i} x_{i, i+1}\right)$ in $\hat{\phi}_{\left(0, \ldots, 0, m_{i}, 0 \ldots, 0\right)}(z)$ is the only place $x_{i, i+1}$ appears. As a result $\sum_{\ell=1}^{i} \gamma_{i, \ell} x_{\ell, i+1}$ only appears in the exponential after applying $\gamma_{i}$. Integrating against $x_{\ell, i+1}$ forces $\gamma_{\ell, i+1}$ to be 0 for $1 \leq \ell \leq i-1$. This also means that $\gamma_{i, i}=1$ as $\gamma \in S L_{n-1}(\mathbb{Z})$. So only the identity coset in the sum over $P_{i}(\mathbb{Z}) \backslash S L_{i}(\mathbb{Z})$ contributes. Finally integrating against either $x_{i+1, i+2}$ or $x_{i-1, i}$ shows the cuspidal contribution is 0 . This completes the proof.

4.5. Partition of type $n=n_{1}+\cdots+n_{r}, n_{r} \geq 2$. The goal of this section to prove the following proposition.

Proposition 4.3. Let $n=n_{1}+\cdots+n_{r}$ be a partition of $n$ in non-increasing order with $n_{r} \geq 2$, we have

$$
\int_{S L_{n}(\mathbb{Z}) \backslash X_{n}} E_{n_{1}, \ldots, n_{r}}\left(z,, \eta, \phi_{1}, \ldots, \phi_{r}\right)\left|E_{n-1,1}(z, s, 1)\right|^{2} d^{*} z=0 .
$$

Proof. By Lemma 4.5, the triple product can be written as

$$
\begin{aligned}
& \int_{S L_{n}(\mathbb{Z}) \backslash X_{n}} E_{n_{1}, \ldots, n_{r}}\left(z, \phi_{1}, \ldots, \phi_{r}, \eta\right) \overline{E_{n-1,1}}(z, 1, s) E_{n-1,1}(z, 1, s) d^{*} z \\
= & \int_{S L_{n-1}(\mathbb{Z}) \backslash X_{n-1}} \int_{0}^{\infty} \int_{(\mathbb{Z} / \mathbb{R})^{n-1}} E_{n_{1}, \ldots, n_{r}}\left(z, \phi_{1}, \ldots, \phi_{r}, \eta\right) \overline{E_{n-1,1}}(z, 1, s) \ell^{-n s}\left(-\frac{n}{n-1}\right) d \bar{x}_{n} \ell^{n} \frac{d \ell}{\ell} d^{*} z^{\prime},
\end{aligned}
$$

where

$\Xi\left(z^{\prime}\right):=\int_{0}^{\infty} \int_{(\mathbb{Z} / \mathbb{R})^{n-1}} E_{n_{1}, \ldots, n_{r}}\left(\bar{x}_{n}, z^{\prime}, \ell, \phi_{1}, \ldots, \phi_{r}, \eta\right) \overline{E_{n-1,1}}\left(\bar{x}_{n}, z^{\prime}, \ell, 1, s\right) \ell^{-n s}\left(-\frac{n}{n-1}\right) d \bar{x}_{n} \ell^{n} \frac{d \ell}{\ell}$

is automorphic.

We have the Fourier expansion:

$$
\begin{aligned}
& \sum_{m_{1}=0}^{\infty} \sum_{m_{2}=0}^{E_{n_{1}, \ldots, n_{r}}}\left(z, \phi_{1}, \ldots, \phi_{r}, \eta\right) \\
& \times \sum_{\gamma_{2} \in P_{1,1} \backslash S L_{2}}^{\prime} \ldots \sum_{m_{m_{1}, \ldots, m_{n-1}}(Y) e}^{\infty} \sum_{m_{\gamma_{n-1}} \in P_{n-2,1} \backslash S L_{n-1}}^{\infty} a_{m_{1}, \ldots, m_{n-1}} \\
& \left.\sum_{1,2}+\cdots m_{n-1} x_{n-1, n}\right)\left.\right|_{\gamma_{2} \cdots \gamma_{n-1}}
\end{aligned}
$$

By Lemma 2.1 we have that

(1) $a_{m_{1}, \ldots, m_{n-2}, 0}=0$, hence, in the Fourier expansion above, the variable $m_{n-1}$ starts at 1.

(2) $a_{m_{1}, \ldots, m_{n-4}, 0,0, m_{n-1}}=0$.

(3) $a_{0, \ldots, 0}=0$.

We now use these properties to simplify $\Xi\left(z^{\prime}\right)$. Notice $m_{n-1} \neq 0$ in the expansion for $E_{n_{1}, \ldots, n_{r}}\left(z, \phi_{1}, \ldots, \phi_{r}, \eta\right)$, so $E_{n_{1}, \ldots, n_{r}}\left(z, \phi_{1}, \ldots, \phi_{r}, \eta\right)$ is orthogonal to all terms with $i<n-1$ in the Fourier expansion of $E_{(n-1,1)}(z, s, 1)$. Thus after executing the $\bar{x}_{n}$ integrals we have

$$
\begin{aligned}
\Xi\left(z^{\prime}\right)= & \int_{0}^{\infty} \sum_{m_{1}=0}^{\infty} \sum_{m_{2}=0}^{\infty} \sum_{\gamma_{2} \in P_{1,1} \backslash S L_{2}}^{\prime} \ldots \sum_{m_{n-1}=1}^{\infty} \sum_{\gamma_{n-1} \in P_{n-2,1} \backslash S L_{n-1}} a_{m_{1}, \ldots, m_{n-1}} b_{m_{n-1}} \\
& \times\left.\left. W_{m_{1}, \ldots, m_{n-1}}\left(Y^{\prime}, \ell\right) e\left(m_{1} x_{1,2}+\cdots m_{n-2} x_{n-2, n-1}\right)\right|_{\gamma_{2} \cdots \gamma_{n-1}} W_{m_{n-1}}^{\prime}\left(Y^{\prime}, \ell\right)\right|_{\gamma_{n-1}}\left(-\frac{n}{n-1}\right) \ell^{n} \frac{d \ell}{\ell} .
\end{aligned}
$$


Here $z^{\prime}=X^{\prime} Y^{\prime}$ in Iwasawa form and we used the simplified notation for the Fourier coefficients of degenerate Eisenstein series:

$$
\begin{aligned}
b_{m_{n-1}} & =\frac{2}{\xi(n s)}\left|m_{n-1}\right|^{\frac{n s}{2}-\frac{1}{2}} \sigma_{-n s+1}\left(\left|m_{n-1}\right|\right) \\
W^{\prime}\left(Y^{\prime}, \ell\right) & =K_{\frac{n s}{2}-\frac{1}{2}}\left(2 \pi\left|m_{n-1}\right| y_{1}\right)\left(y_{1}^{n-1} y_{2}^{n-2} \cdots y_{n-1}\right)^{s} y_{1}^{-\frac{n s}{2}+\frac{1}{2}} \\
& =K_{\frac{n s}{2}-\frac{1}{2}}\left(2 \pi\left|m_{n-1}\right| y_{1}\right) \ell^{-n s} y_{1}^{-\frac{n s}{2}+\frac{1}{2}} .
\end{aligned}
$$

This corresponds to the term $\hat{\phi}_{\left(0, \ldots, 0, m_{n-1}\right)}$ in Theorem 3.1. It is important to note that $\ell$ is invariant under the action of $\gamma_{2}, \ldots, \gamma_{n-1}$, so we may move the integral inside all the summations:

$$
\begin{aligned}
\Xi\left(z^{\prime}\right)= & \sum_{m_{1}=0}^{\infty} \sum_{m_{2}=0}^{\infty} \sum_{\gamma_{2} \in P_{1,1} \backslash S L_{2}}^{\prime} \cdots \sum_{m_{n-1}=1}^{\infty} \sum_{\gamma_{n-1} \in P_{n-2,1} \backslash S L_{n-1}} a_{m_{1}, \ldots, m_{n-1}} b_{m_{n-1}} \\
& \times\left.\left.\int_{0}^{\infty} W_{m_{1}, \ldots, m_{n-1}}\left(Y^{\prime}, \ell\right) e\left(m_{1} x_{1,2}+\cdots m_{n-2} x_{n-2, n-1}\right)\right|_{\gamma_{2} \cdots \gamma_{n-1}} W_{m_{n-1}}^{\prime}\left(Y^{\prime}, \ell\right)\right|_{\gamma_{n-1}}\left(-\frac{n}{n-1}\right) \ell^{n} \frac{d \ell}{\ell} .
\end{aligned}
$$

It's tempting to conclude here that $\Xi\left(z^{\prime}\right)$ is an automorphic function without constant term, so

$\int_{S L_{n-1} \backslash X_{n-1}} \Xi\left(z^{\prime}\right) d^{*} z^{\prime}$ must be 0. However this is not the correct form of Fourier expansion for $\Xi\left(z^{\prime}\right)$.

Note that $\Xi\left(z^{\prime}\right)$ is a function on $S L_{n-1} \backslash X_{n-1}$. The correct form of Fourier expansion looks like

$$
\sum_{m_{1}=0}^{\infty} \sum_{m_{2}=0}^{\infty} \sum_{\gamma_{2} \in P_{1,1} \backslash S L_{2}}^{\prime} \ldots \sum_{m_{n-2}=0}^{\infty} \sum_{\gamma_{n-2} \in P_{n-3,1} \backslash S L_{n-2}}^{\prime} a_{\left(m_{1}, \ldots, m_{n-2}\right)} f_{\left(m_{1}, \ldots, m_{n-2}\right)}(y) e\left(m_{1} x_{1,2}+\ldots+m_{n-2} x_{n-2, n-1}\right) .
$$

In our expansion of $\Xi\left(z^{\prime}\right)$, we have an sum over $P_{n-2,1} \backslash S L_{n-1}$. But this allows us to unfold again.

$$
\begin{aligned}
& \int_{S L_{n-1} \backslash X_{n-1}} \Xi\left(z^{\prime}\right) d^{*} z^{\prime} \\
= & \int_{P_{n-2,1} \backslash X_{n-1}} \sum_{m_{1}=0}^{\infty} \sum_{m_{2}=0}^{\infty} \sum_{\gamma_{2} \in P_{1,1} \backslash S L_{2}}^{\prime} \cdots \sum_{m_{n-2}=0}^{\infty} \sum_{\gamma_{n-2} \in P_{n-3,1} \backslash S L_{n-2}}^{\prime} \sum_{m_{n-1}=1}^{\infty} a_{m_{1}, \ldots, m_{n-1}} b_{m_{n-1}} \\
& \times\left.\int_{0}^{\infty} W_{m_{1}, \ldots, m_{n-1}}\left(Y^{\prime}, \ell\right) e\left(m_{1} x_{1,2}+\cdots m_{n-2} x_{n-2, n-1}\right)\right|_{\gamma_{2} \cdots \gamma_{n-2}} W_{m_{n-1}}^{\prime}\left(Y^{\prime}, \ell\right)\left(-\frac{n}{n-1}\right) \ell^{n} \frac{d \ell}{\ell} d^{*} z^{\prime}
\end{aligned}
$$

Now suppose $F(z)$ is an automorphic function on $S L_{n}(\mathbb{Z}) \backslash X_{n}$ in the form of $F(z)=\left.\sum_{\gamma \in P_{n-1,1} \backslash S L_{n}(\mathbb{Z})} G(z)\right|_{\gamma}$. First of all, for this definition to make sense, we need $G(z)$ to be invariant under the group $P_{n-1,1}$. (This is clearly the case for $\Xi\left(z^{\prime}\right)$.) Then

$$
\begin{aligned}
& \int_{S L_{n}(\mathbb{Z}) \backslash X_{n}} F(z) d^{*} z \\
& =\int_{P_{n-1,1} \backslash X_{n}} G(z) d^{*} z \\
& =\int_{S L_{n-1}(\mathbb{Z}) \backslash X_{n-1}} \int_{0}^{\infty} \int_{(\mathbb{Z} / \mathbb{R})^{n-1}} G\left(\left(\begin{array}{cc}
I_{n-1} & \bar{x}_{n} \\
& 1
\end{array}\right) \cdot\left(\begin{array}{ll}
z^{\prime} & \\
& 1
\end{array}\right) \cdot\left(\begin{array}{ll}
\ell^{-\frac{1}{n-1}} \cdot I_{n-1} & \\
&
\end{array}\right)\right) \\
& \times\left(-\frac{n}{n-1}\right) d \bar{x}_{n} \ell^{n} \frac{d \ell}{\ell} d^{*} z^{\prime} .
\end{aligned}
$$

We must verify that

$$
G^{\prime}\left(z^{\prime}\right):=\int_{0}^{\infty} \int_{(\mathbb{Z} / \mathbb{R})^{n-1}} G\left(\left(\begin{array}{cc}
I_{n-1} & \bar{x}_{n} \\
& 1
\end{array}\right) \cdot\left(\begin{array}{ll}
z^{\prime} & \\
& 1
\end{array}\right) \cdot\left(\begin{array}{ll}
\ell^{-\frac{1}{n-1}} \cdot I_{n-1} & \\
&
\end{array}\right)\right)\left(-\frac{n}{n-1}\right) d \bar{x}_{n} \ell^{n} \frac{d \ell}{\ell}
$$


is automorphic. Indeed,

$$
\begin{aligned}
& G^{\prime}\left(\gamma z^{\prime}\right) \\
& =\int_{0}^{\infty} \int_{(\mathbb{Z} / \mathbb{R})^{n-1}} W\left(\left(\begin{array}{cc}
I_{n-1} & \bar{x}_{n} \\
& 1
\end{array}\right) \cdot\left(\begin{array}{ll}
\gamma & \\
& 1
\end{array}\right) \cdot\left(\begin{array}{cc}
z^{\prime} & \\
& 1
\end{array}\right) \cdot\left(\begin{array}{cc}
\ell^{-\frac{1}{n-1}} \cdot I_{n-1} & \\
&
\end{array}\right)\right)\left(\begin{array}{ll}
-\frac{n}{n-1} &
\end{array}\right) d \bar{x}_{n} \ell^{n} \frac{d \ell}{\ell}
\end{aligned}
$$

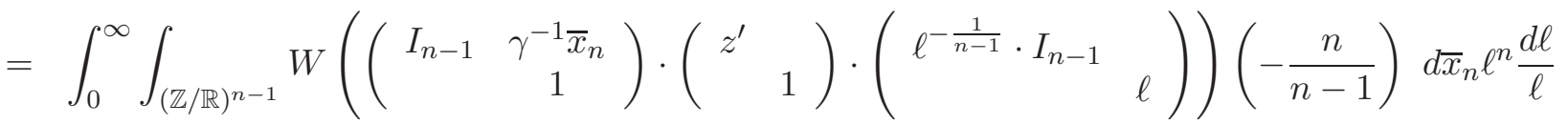

$$
\begin{aligned}
& =\int_{0}^{\infty} \int_{(\mathbb{Z} / \mathbb{R})^{n-1}} W\left(\left(\begin{array}{cc}
I_{n-1} & \bar{x}_{n} \\
& 1
\end{array}\right) \cdot\left(\begin{array}{ll}
z^{\prime} & \\
& 1
\end{array}\right) \cdot\left(\begin{array}{ll}
\ell^{-\frac{1}{n-1}} \cdot I_{n-1} & \\
&
\end{array}\right)\right)\left(-\frac{n}{n-1}\right) d \bar{x}_{n} \ell^{n} \frac{d \ell}{\ell} \\
& =G^{\prime}\left(z^{\prime}\right) \text {. } \\
& \int_{S L_{n-1} \backslash X_{n-1}} \Xi\left(z^{\prime}\right) d^{*} z^{\prime} \\
& =\int_{P_{n-2,1} \backslash X_{n-1}} \sum_{m_{1}=0}^{\infty} \sum_{m_{2}=0}^{\infty} \sum_{\gamma_{2} \in P_{1,1} \backslash S L_{2}}^{\prime} \ldots \sum_{m_{n-2}=0}^{\infty} \sum_{\gamma_{n-2} \in P_{n-3,1} \backslash S L_{n-2}}^{\prime} \sum_{m_{n-1}=1}^{\infty} a_{m_{1}, \ldots, m_{n-1}} b_{m_{n-1}} \\
& \times\left.\int_{0}^{\infty} W_{m_{1}, \ldots, m_{n-1}}\left(Y^{\prime}, \ell\right) e\left(m_{1} x_{1,2}+\cdots m_{n-2} x_{n-2, n-1}\right)\right|_{\gamma_{2} \cdots \gamma_{n-2}} W_{m_{n-1}}^{\prime}\left(Y^{\prime}, \ell\right)\left(-\frac{n}{n-1}\right) \ell^{n} \frac{d \ell}{\ell} d^{*} z^{\prime} \\
& =\int_{S L_{n-2} \backslash X_{n-2}} \sum_{m_{1}=0}^{\infty} \sum_{m_{2}=0}^{\infty} \sum_{\gamma_{2} \in P_{1,1} \backslash S L_{2}}^{\prime} \ldots \sum_{m_{n-3}=1}^{\infty} \sum_{\gamma_{n-3} \in P_{n-4,1} \backslash S L_{n-3}} \sum_{m_{n-1}=1}^{\infty} a_{m_{1}, \ldots, m_{n-3}, 0, m_{n-1}} b_{m_{n-1}} \\
& \times\left.\int_{0}^{\infty} \int_{0}^{\infty} W_{m_{1}, \ldots, m_{n-3}, 0, m_{n-1}}\left(Y^{\prime \prime}, \ell^{\prime}, \ell\right) e\left(m_{1} x_{1,2}+\cdots+m_{n-3} x_{n-3, n-2}\right)\right|_{\gamma_{2} \cdots \gamma_{n-3}} \\
& \times W_{m_{n-1}}^{\prime}\left(\ell^{\prime}, \ell\right)\left(-\frac{n}{n-1}\right) \ell^{n} \frac{d \ell}{\ell}\left(-\frac{n-1}{n-2}\right) \ell^{\prime n} \frac{d \ell^{\prime}}{\ell^{\prime}} d^{*} z^{\prime}
\end{aligned}
$$

with

$$
z^{\prime}=\left(\begin{array}{cc}
I_{n-2} & \bar{x}_{n-1} \\
& 1
\end{array}\right) \cdot\left(\begin{array}{ll}
z^{\prime \prime} & \\
& 1
\end{array}\right) \cdot\left(\begin{array}{ll}
\ell^{-\frac{1}{n-2}} \cdot I_{n-2} & \\
& \\
& \ell^{\prime}
\end{array}\right)
$$

and $\ell^{\prime}=\left(y_{2}^{n-2} \cdots y_{n-1}\right)^{-\frac{1}{n-1}}$.

Here we also note that $W^{\prime}\left(Y^{\prime}, \ell\right)$ is really a function of $y_{1}$ and $\ell$. But $y_{1}=\ell^{-\frac{n}{n-1}} \ell^{\prime}$ so $W^{\prime}\left(Y^{\prime}, \ell\right)$ is a function of $\ell$ and $\ell^{\prime}$. And $\ell$ and $\ell^{\prime}$ are invariant under the action of $\gamma_{2}, \ldots, \gamma_{n-3}$.

Finally,

$$
\begin{aligned}
& \sum_{m_{1}=0}^{\infty} \sum_{m_{2}=0}^{\infty} \sum_{\gamma_{2} \in P_{1,1} \backslash S L_{2}}^{\prime} \ldots \sum_{m_{n-3}=1}^{\infty} \sum_{\gamma_{n-3} \in P_{n-4,1} \backslash S L_{n-3}} \sum_{m_{n-1}=1}^{\infty} a_{m_{1}, \ldots, m_{n-3}, 0, m_{n-1}} b_{m_{n-1}} \\
& \times \int_{0}^{\infty} \int_{0}^{\infty} W_{m_{1}, \ldots, m_{n-3}, 0, m_{n-1}}\left(Y^{\prime \prime}, \ell^{\prime}, \ell\right) W_{m_{n-1}}^{\prime}\left(\ell^{\prime}, \ell\right)\left(-\frac{n}{n-1}\right) \ell^{n} \frac{d \ell}{\ell}\left(-\frac{n-1}{n-2}\right) \ell^{\prime n} \frac{d \ell^{\prime}}{\ell^{\prime}} \\
& \times\left. e\left(m_{1} x_{1,2}+\cdots+m_{n-3} x_{n-3, n-2}\right)\right|_{\gamma_{2} \cdots \gamma_{n-3}}
\end{aligned}
$$

is in proper Fourier expansion form and is automorphic without constant term. Thus the integral over $S L_{n-2} \backslash X_{n-2}$ is 0 . 


\section{Main Term From Minimal Parabolic Contribution}

The purpose of this section is to prove the following theorem.

\section{Theorem 5.1.}

$$
\begin{aligned}
& \int_{S L_{n}(\mathbb{Z}) \backslash X_{n}} E_{(1, \ldots, 1)}(z, \eta)\left|E_{(n-1,1)}\left(z, \frac{1}{2}+i t, 1\right)\right|^{2} d^{*} z \\
= & \frac{2}{\xi(n)} \log (t)\left(\int_{S L_{n}(\mathbb{Z}) \backslash X_{n}} E_{(1, \ldots, 1)}(z, \eta) d^{*} z\right)+O_{n, \eta}(1),
\end{aligned}
$$

as $t \rightarrow \infty$.

We begin the proof by evaluating the integral on the right hand side.

Proposition 5.1.

$$
\int_{S L_{n}(\mathbb{Z}) \backslash X_{n}} E_{(1, \ldots, 1)}(z, \eta) d^{*} z=\frac{1}{n^{n-2}} \tilde{\eta}\left(\frac{2}{n}, \ldots, \frac{2}{n}\right)
$$

Proof. Recall that the incomplete Eisenstein series associated to the minimal parabolic subgroup is given by

$$
E_{(1, \ldots, 1)}(z, \eta)=\left.\sum_{\gamma \in P_{1, \ldots, 1}(\mathbb{Z}) \backslash S L_{n}(\mathbb{Z})} \eta\left(\prod_{j=1}^{n-1} y_{j}^{b_{j, 1}}, \prod_{j=1}^{n-1} y_{j}^{b_{j, 2}}, \ldots, \prod_{j=1}^{n-1} y_{j}^{b_{j, n-1}}\right)\right|_{\gamma}
$$

where

$$
b_{j, k}= \begin{cases}j k & \text { if } j+k \leq n, \\ (n-j)(n-k) & \text { if } j+k \geq n .\end{cases}
$$

We can unfold with respect to the sum over $P_{1, \ldots, 1}(\mathbb{Z}) \backslash S L_{n}(\mathbb{Z})$ :

$$
\begin{aligned}
& \int_{S L_{n}(\mathbb{Z}) \backslash X_{n}} E_{(1, \ldots, 1)}(z, \eta) d^{*} z \\
= & \int_{P_{1, \ldots, 1}(\mathbb{Z}) \backslash X_{n}} \eta\left(\prod_{j=1}^{n-1} y_{j}^{b_{j, 1}}, \prod_{j=1}^{n-1} y_{j}^{b_{j, 2}}, \ldots, \prod_{j=1}^{n-1} y_{j}^{b_{j, n-1}}\right) d^{*} z \\
= & \int_{\left(\mathbb{R}^{+}\right)^{n-1}} \int_{N_{\min }(\mathbb{Z}) \backslash N_{\min }(\mathbb{R})} \eta\left(\prod_{j=1}^{n-1} y_{j}^{b_{j, 1}}, \prod_{j=1}^{n-1} y_{j}^{b_{j, 2}}, \ldots, \prod_{j=1}^{n-1} y_{j}^{b_{j, n-1}}\right) \prod_{1 \leq \alpha<\beta \leq n} d x_{\alpha, \beta} \prod_{\ell=1}^{n-1} y_{\ell}^{-\ell(n-\ell)-1} d y_{\ell} \\
= & \int_{\left(\mathbb{R}^{+}\right)^{n-1}} \eta\left(\prod_{j=1}^{n-1} y_{j}^{b_{j, 1}}, \prod_{j=1}^{n-1} y_{j}^{b_{j, 2}}, \ldots, \prod_{j=1}^{n-1} y_{j}^{b_{j, n-1}}\right) \prod_{\ell=1}^{n-1} y_{\ell}^{-\ell(n-\ell)-1} d y_{\ell} .
\end{aligned}
$$

At this moment, we make a change of variables:

$$
w_{k}=\prod_{j=1}^{n-1} y_{j}^{b_{j, k}} \quad \text { for } 1 \leq k \leq n-1
$$

One can easily show that

$$
y_{j}=\left(\frac{w_{n-j}^{2}}{w_{n-j-1} w_{n-j+1}}\right)^{\frac{1}{n}} \quad \text { for } 1 \leq j \leq n-1
$$


where we set $w_{0}=w_{n}=1$. Then we can calculate the Jacobian of this change of variables

$$
J=\frac{1}{n^{n-2}}\left(w_{1} w_{n-1}\right)^{-\frac{n-1}{n}} \prod_{k=2}^{n-2} w_{k}^{-1} .
$$

At the same time,

$$
\prod_{\ell=1}^{n-1} y_{\ell}^{-\ell(n-\ell)-1}=\left(w_{1} w_{n-1}\right)^{-\frac{3}{n}} \prod_{k=2}^{n-2} w_{k}^{-\frac{2}{n}}
$$

Thus

$$
\begin{aligned}
& \int_{S L_{n}(\mathbb{Z}) \backslash X_{n}} E_{(1, \ldots, 1)}(z, \eta) d^{*} z \\
= & \int_{\left(\mathbb{R}^{+}\right)^{n-1}} \eta\left(w_{1}, \ldots, w_{n-1}\right) \frac{1}{n^{n-2}} \prod_{k=1}^{n-1} w_{k}^{-\frac{2}{n}} \prod_{\ell=1}^{n-1} \frac{d w_{\ell}}{w_{\ell}} \\
= & \frac{1}{n^{n-2}} \tilde{\eta}\left(\frac{2}{n}, \ldots, \frac{2}{n}\right)
\end{aligned}
$$

as desired.

To prove Theorem 5.1, we use the Fourier expansion of the degenerate Eisenstein series from Theorem 3.1. We cross multiply the Fourier expansion to open the square. There are four type of terms that we will evaluate separately:

(1)

$$
\Delta_{1}(z, t):=\left|\hat{\phi}_{(0, \ldots, 0)}\left(z, \frac{1}{2}+i t\right)\right|^{2}
$$

$$
\Delta_{2}(z, t):=\left|\sum_{m_{1} \neq 0} \hat{\phi}_{\left(m_{1}, 0, \ldots, 0\right)}\left(z, \frac{1}{2}+i t\right)\right|^{2} ;
$$

(3) For $2 \leq k \leq n-1$

$$
\Delta_{3, k}(z, t):=\left|\sum_{\gamma_{i} \in P_{i}(\mathbb{Z}) \backslash S L_{k}(\mathbb{Z})} \sum_{m_{k}=1}^{\infty} \hat{\phi}_{\left(0, \ldots, 0, m_{k}, 0 \ldots, 0\right)}\left(\left(\begin{array}{cc}
\gamma_{i} & \\
& I_{n-i}
\end{array}\right) z, \frac{1}{2}+i t\right)\right|^{2} ;
$$

(4) All cross terms.

Lemma 5.1. All cross terms vanish.

Proof. A typical term after opening the square has the form

$\sum_{\gamma_{i} \in P_{i}(\mathbb{Z}) \backslash S L_{i}(\mathbb{Z})} \sum_{m_{i}=1}^{\infty} \hat{\phi}_{\left(0, \ldots, 0, m_{i}, 0 \ldots, 0\right)}\left(\left(\begin{array}{cc}\gamma_{i} & \\ & I_{n-i}\end{array}\right) z\right) \sum_{\gamma_{j} \in P_{j}(\mathbb{Z}) \backslash S L_{j}(\mathbb{Z})} \sum_{m_{j}=1}^{\infty} \overline{\hat{\phi}}_{\left(0, \ldots, 0, m_{j}, 0 \ldots, 0\right)}\left(\left(\begin{array}{ll}\gamma_{j} & \\ & I_{n-j}\end{array}\right) z\right)$.

If $i \neq j$, without loss of generality we can assume $i>j$. Then integrating against the variables $x_{1, k+1}, \ldots, x_{k-1, k+1}$ forces $\gamma_{i}$ to be the identity coset. Then the this cross term vanishes after integrating against $x_{k, k+1}$. 
Lemma 5.2.

$$
\int_{S L_{n}(\mathbb{Z}) \backslash X_{n}} E_{(1, \ldots, 1)}(z, \eta) \Delta_{1}(z, t) d^{*} z=O(1)
$$

Proof.

$$
\Delta_{1}(z, t)=\left|\sum_{k=0}^{n-1} \frac{2 \xi\left(k+1-\frac{n}{2}+i n t\right)}{\xi\left(\frac{n}{2}+i n t\right)}\left(y_{1} y_{2}^{2} \cdots y_{n-k-1}^{n-k-1}\right)^{\frac{1}{2}-i t}\left(y_{n-k}^{k} y_{n-(k-1)}^{k-1} \cdots y_{n-1}\right)^{\frac{1}{2}+i t}\right|^{2}
$$

First we treat diagonal terms:

$$
\begin{aligned}
& \int_{S L_{n}(\mathbb{Z}) \backslash X_{n}} E_{(1, \ldots, 1)}(z, \eta) \sum_{k=0}^{n-1} \frac{\left|\xi\left(k+1-\frac{n}{2}+i n t\right)\right|^{2}}{\left|\xi\left(\frac{n}{2}+i n t\right)\right|^{2}}\left(y_{1} y_{2}^{2} \cdots y_{n-k-1}^{n-k-1}\right)\left(y_{n-k}^{k} y_{n-(k-1)}^{k-1} \cdots y_{n-1}\right) d^{*} z \\
= & \sum_{k=0}^{n-1} \frac{\left|\xi\left(k+1-\frac{n}{2}+i n t\right)\right|^{2}}{\left|\xi\left(\frac{n}{2}+i n t\right)\right|^{2}} \int_{0}^{\infty} \cdots \int_{0}^{\infty} \eta\left(\prod_{j=1}^{n-1} y_{j}^{b_{j, 1}}, \prod_{j=1}^{n-1} y_{j}^{b_{j, 2}}, \ldots, \prod_{j=1}^{n-1} y_{j}^{b_{j, n-1}}\right) \\
& \times\left(y_{1} y_{2}^{2} \cdots y_{n-k-1}^{n-k-1}\right)\left(y_{n-k}^{k} y_{n-(k-1)}^{k-1} \cdots y_{n-1}\right) \prod_{\ell=1}^{n-1} y_{\ell}^{-\ell(n-\ell)-1} d y_{\ell} \\
= & \sum_{k=0}^{n-1} \frac{\left|\xi\left(k+1-\frac{n}{2}+i n t\right)\right|^{2}}{\left|\xi\left(\frac{n}{2}+i n t\right)\right|^{2}} c_{k, \eta}
\end{aligned}
$$

for some constants $c_{k, \eta}$. We will not calculate these constants as they do not contribute to the main term. For $k=0, n-1$, we get a contribution of $O(1)$. By Stirling's formula the rest will contribute $O\left(t^{-1}\right)$.

For off-diagonal terms, some powers of $y$ will be imaginary unlike the diagonal terms. We can then use integration by parts repeatedly and get a contribution of $O\left(t^{-2016}\right)$, say.

\section{Lemma 5.3.}

$$
\begin{aligned}
& \int_{S L_{n}(\mathbb{Z}) \backslash X_{n}} E_{(1, \ldots, 1)}(z, \eta) \Delta_{2}(z, t) d^{*} z \\
= & \frac{2}{\left|\xi\left(\frac{n}{2}+i n t\right)\right|^{2}} \int_{\left(\mathbb{R}^{+}\right)^{n-2}} \frac{1}{(2 \pi i)^{n-1}} \int_{(2)} \ldots \int_{(2)} \tilde{\eta}(\nu) \\
& \times \frac{\xi\left(\sum_{j=1}^{n-1} b_{n-1, j} \nu_{j}\right) \xi\left(\sum_{j=1}^{n-1} b_{n-1, j} \nu_{j}-\frac{n}{2}+1+i n t\right)}{\xi\left(2 \sum_{j=1}^{n-1} b_{n-1, j} \nu_{j}-n+2\right)} \\
& \times \xi\left(\sum_{j=1}^{n-1} b_{n-1, j} \nu_{j}-\frac{n}{2}+1-i n t\right) \xi\left(\sum_{j=1}^{n-1} b_{n-1, j} \nu_{j}-n+2\right) \\
& \times\left(y_{1} y_{2}^{2} \cdots y_{n-2}^{n-2}\right) \prod_{\ell=1}^{n-1} d \nu_{\ell} \prod_{k=1}^{n-2} \frac{d y_{k}}{y_{k}^{k(n-k)+1}}
\end{aligned}
$$


Proof.

$$
\begin{aligned}
& \int_{S L_{n}(\mathbb{Z}) \backslash X_{n}} E_{(1, \ldots, 1)}(z, \eta) \Delta_{2}(z, t) d^{*} z \\
= & \int_{S L_{n}(\mathbb{Z}) \backslash X_{n}} E_{(1, \ldots, 1)}(z, \eta)\left|\sum_{m_{1} \neq 0} \hat{\phi}_{\left(m_{1}, 0, \ldots, 0\right)}\left(z, \frac{1}{2}+i t\right)\right|^{2} d^{*} z \\
= & \frac{8}{\left|\xi\left(\frac{n}{2}+i n t\right)\right|^{2}} \int_{\left(\mathbb{R}^{+}\right)^{n-1}} \int_{N_{\min (\mathbb{Z})} \backslash N_{\min (\mathbb{R})}} \frac{1}{(2 \pi i)^{n-1}} \int_{(2)} \cdots \int_{(2)} \tilde{\eta}(\nu)\left(\prod_{i=1}^{n-1} y_{i}^{\sum_{j=1}^{n-1} b_{i, j} \nu_{j}}\right) \\
& \times \sum_{m_{1}=1}^{\infty} m_{1}^{1-\frac{n}{2}} \sigma_{\frac{n}{2}-1-i n t}\left(m_{1}\right) \sigma_{\frac{n}{2}-1+i n t}\left(m_{1}\right) K_{\frac{1}{2}-\frac{n}{4}+\frac{i n t}{2}}\left(2 \pi m_{1} y_{n-1}\right) K_{\frac{1}{2}-\frac{n}{4}-\frac{i n t}{2}}\left(2 \pi m_{1} y_{n-1}\right) \\
& \times\left(y_{1} y_{2}^{2} \cdots y_{n-2}^{n-2}\right) y_{n-1}^{\frac{n}{2}} \prod_{\ell=1}^{n-1} d \nu_{\ell} \prod_{k=1}^{n-1} \frac{d y_{k}}{y_{k}^{k(n-k)+1}}
\end{aligned}
$$

The Lemma is then proved by applying Lemma 4.2 to convert the divisor sum into Zeta functions and 4.1 to convert K-Bessel integrals to Gamma functions.

\section{Lemma 5.4.}

$$
\begin{aligned}
& \int_{S L_{n}(\mathbb{Z}) \backslash X_{n}} E_{(1, \ldots, 1)}(z, \eta) \Delta_{3, k}(z, t) d^{*} z \\
= & \frac{2}{\left|\xi\left(\frac{n}{2}+i n t\right)\right|^{2}} \int_{\left(\mathbb{R}^{+}\right)^{n-2}} \frac{1}{(2 \pi i)^{n-1}} \int_{(2)} \cdots \int_{(2)} \tilde{\eta}(\nu) \\
& \times \sum_{h=0}^{k-1} \frac{\xi\left((1-k)(n-k)+\sum_{j=1}^{n-1} b_{n-k, j} \nu_{j}\right) \xi\left((1-k)(n-k)+\sum_{j=1}^{n-1} b_{n-k, j} \nu_{j}-\frac{n}{2}+k+i n t\right)}{\xi\left(2(1-k)(n-k)+2 \sum_{j=1}^{n-1} b_{n-k, j} \nu_{j}-n+2 k\right)} \\
& \times \xi\left((1-k)(n-k)+\sum_{j=1}^{n-1} b_{n-k, j} \nu_{j}-\frac{n}{2}+k-i n t\right) \xi\left(\sum_{j=1}^{n-1} b_{n-k, j} \nu_{j}-k(n-k)+h+1\right) \\
& \times \prod_{i=1}^{n-k-1} y_{i}^{\sum_{j=1}^{n-1} b_{i, j} \nu_{j}+i-i(n-i)} \prod_{i=n-k+1}^{n-h-1} y_{i}^{k(n-k)-i(n-i)+\sum_{j=1}^{n-1}\left(b_{i, j}-b_{n-k, j}\right) \nu_{j}} \prod_{i=n-h}^{n-1} y_{i}^{\sum_{j=1}^{n-1} b_{i, j} \nu_{j}+n-i-i(n-i)} \\
& \prod_{\ell=1}^{n-1} d \nu_{\ell} \prod_{i=1}^{n-1} \frac{d y_{i}}{y_{i}}
\end{aligned}
$$

Proof. By Lemma 3.3, as $\gamma_{i}$ maps $x_{i, i+1}$ to $a_{1} x_{1, i+1}+a_{2} x_{2, i+1}+\cdots a_{i} x_{i, i+1}$ where the primitive vector $\left(a_{1}, \ldots, a_{i}\right)$ is the bottom row of $\gamma_{i}$. Now integrating the exponential factor

$$
e\left(m_{i}\left(a_{1} x_{1, i+1}+a_{2} x_{2, i+1}+\cdots a_{i} x_{i, i+1}\right)-m_{i}^{\prime}\left(a_{1}^{\prime} x_{1, i+1}+a_{2}^{\prime} x_{2, i+1}+\cdots a_{i}^{\prime} x_{i, i+1}\right)\right)
$$

in the diagonal term forces $m_{i}=m_{i}^{\prime}$ and $\gamma_{i}=\gamma_{i}^{\prime}$ up to a sign. Thus the $k$-th $(2 \leq k \leq n-1)$ diagonal term $\Xi_{k}$ reads with $s=\frac{1}{2}+i t$ :

$$
\begin{aligned}
\Xi_{k}:= & \frac{8}{\xi\left(\frac{n}{2}+i n t\right) \xi\left(\frac{n}{2}-i n t\right)} \sum_{\gamma_{k} \in P_{k}(\mathbb{Z}) \backslash S L_{k}(\mathbb{Z})} \sum_{m_{k}=1}^{\infty}\left|m_{k}\right|^{k-\frac{n}{2}} \sigma_{\frac{n}{2}-k-i n t}\left(\left|m_{k}\right|\right) \sigma_{\frac{n}{2}-k+i n t}\left(\left|m_{k}\right|\right) \\
& \times K_{\frac{k}{2}-\frac{n}{4}+\frac{i n t}{2}}\left(2 \pi\left|m_{k}\right| y_{n-k}^{\prime}\right) K_{\frac{k}{2}-\frac{n}{4}-\frac{i n t}{2}}\left(2 \pi\left|m_{k}\right| y_{n-k}^{\prime}\right)\left(y_{1} y_{2}^{2} \cdots y_{n-k-1}^{n-k-1}\right)\left(y_{n-k}^{k} y_{n-(k-1)}^{k-1} \cdots y_{n-1}\right) y_{n-k}^{\prime \frac{n}{2}-k}
\end{aligned}
$$


Putting all terms together, we get

$$
\Omega_{k}:=\int_{\left(\mathbb{R}^{+}\right)^{n-1}} \int_{N_{\min (\mathbb{Z})} \backslash N_{\min (\mathbb{R})}} \frac{1}{(2 \pi i)^{n-1}} \int_{(2)} \cdots \int_{(2)} \prod_{i=1}^{n-1} y_{i}^{\sum_{j=1}^{n-1} b_{i, j} \nu_{j}} \Xi_{k} \prod_{\ell=1}^{n-1} d \nu_{\ell} \prod_{1 \leq i<j \leq n-1} d x_{i, j} \prod_{k=1}^{n-1} \frac{d y_{k}}{y_{k}^{k(n-k)+1}} .
$$

We now make the change of variable $\left|m_{k}\right| y_{n-k}^{\prime} \mapsto y_{n-k}$ and let $\Theta_{k}=\left(b_{1}^{2}+b_{2}^{2}+\cdots+b_{k}^{2}\right)^{\frac{1}{2}}$. So we have

$$
\begin{aligned}
\Omega_{k}= & \frac{8}{\xi\left(\frac{n}{2}+i n t\right) \xi\left(\frac{n}{2}-i n t\right)} \int_{\left(\mathbb{R}^{+}\right)^{n-1}} \int_{N_{\min (\mathbb{Z})} \backslash N_{\min (\mathbb{R})}} \frac{1}{(2 \pi i)^{n-1}} \int_{(2)} \cdots \int_{(2)} \tilde{\eta}(\nu)\left(\prod_{i=1}^{n-1} y_{i}^{\sum_{j=1}^{n-1} b_{i, j} \nu_{j}}\right) \\
& \times \sum_{\gamma_{k} \in P_{k}(\mathbb{Z}) \backslash S L_{k}(\mathbb{Z})} \sum_{m_{k}=1}^{\infty}\left|m_{k}\right|^{k-\frac{n}{2}} \sigma_{\frac{n}{2}-k-i n t}\left(\left|m_{k}\right|\right) \sigma_{\frac{n}{2}-k+i n t}\left(\left|m_{k}\right|\right)\left(\left|m_{k}\right| \Theta_{k}\right)^{-\sum_{j=1}^{n-1} b_{n-k, j} \nu_{j}-k+k(n-k)}\left|m_{k}\right|^{k-\frac{n}{2}} \\
& \times K_{\frac{k}{2}-\frac{n}{4}+\frac{i n t}{2}}\left(2 \pi y_{n-k}\right) K_{\frac{k}{2}-\frac{n}{4}-\frac{i n t}{2}}\left(2 \pi y_{n-k}\right)\left(y_{1} y_{2}^{2} \cdots y_{n-k-1}^{n-k-1}\right)\left(y_{n-k}^{k} y_{n-(k-1)}^{k-1} \cdots y_{n-1}\right) y_{n-k}^{\frac{n}{2}-k} \\
& \prod_{\ell=1}^{n-1} d \nu_{\ell} \prod_{1 \leq i<j \leq n-1} d x_{i, j} \prod_{k=1}^{n-1} \frac{d y_{k}}{y_{k}^{k(n-k)+1}} .
\end{aligned}
$$

We use the identity

$$
\sum_{n=1} \frac{\sigma_{a}(n) \sigma_{b}(n)}{n^{s}}=\frac{\zeta(s) \zeta(s-a) \zeta(s-b) \zeta(s-a-b)}{\zeta(2 s-a-b)}
$$

to convert the sum over $m_{k}$ to $\zeta$ functions:

$$
\begin{aligned}
& \sum_{m_{k}=1}^{\infty} \frac{\sigma_{\frac{n}{2}-k-i n t}\left(\left|m_{k}\right|\right) \sigma_{\frac{n}{2}-k+i n t}\left(\left|m_{k}\right|\right)}{\left|m_{k}\right|^{n-k-k(n-k)+\sum_{j=1}^{n-1} b_{n-k, j} \nu_{j}}} \\
= & \frac{\zeta\left((1-k)(n-k)+\sum_{j=1}^{n-1} b_{n-k, j} \nu_{j}\right) \zeta\left((1-k)(n-k)+\sum_{j=1}^{n-1} b_{n-k, j} \nu_{j}-\frac{n}{2}+k+i n t\right)}{\zeta\left(2(1-k)(n-k)+2 \sum_{j=1}^{n-1} b_{n-k, j} \nu_{j}-n+2 k\right)} \\
& \times \zeta\left((1-k)(n-k)+\sum_{j=1}^{n-1} b_{n-k, j} \nu_{j}-\frac{n}{2}+k-i n t\right) \zeta\left((1-k)(n-k)+\sum_{j=1}^{n-1} b_{n-k, j} \nu_{j}-n+2 k\right) .
\end{aligned}
$$

Now we notice that

$$
\sum_{\gamma_{k} \in P_{k}(\mathbb{Z}) \backslash S L_{k}(\mathbb{Z})} \Theta_{k}^{-\sum_{j=1}^{n-1} b_{n-k, j} \nu_{j}-k+k(n-k)}
$$

is just a degenerate Eisenstein series. Integrating over $x$ simply gives the constant term. It reads

$$
\sum_{h=0}^{k-1} \frac{2 \xi\left(\sum_{j=1}^{n-1} b_{n-k, j} \nu_{j}-k(n-k)+h+1\right)\left(y_{(n-k)+1} \cdots y_{(n-k)+(k-h-1)^{+}}\right)^{-\sum_{j=1}^{n-1} b_{n-k, j} \nu_{j}+k(n-k)-h-1}}{\xi\left(\sum_{j=1}^{n-1} b_{n-k, j} \nu_{j}-k(n-k)+k\right)\left(y_{(n-k)+1}^{k-h-2} \cdots y_{(n-k)+(k-h-2)^{+}}\right)}
$$

Now we use the identity

$$
\int_{0}^{\infty} K_{\mu}(y) K_{\nu}(y) y^{s} \frac{d y}{y}=2^{s-3} \frac{\Gamma\left(\frac{s+\mu+\nu}{2}\right) \Gamma\left(\frac{s+\mu-\nu}{2}\right) \Gamma\left(\frac{s-\mu+\nu}{2}\right) \Gamma\left(\frac{s-\mu-\nu}{2}\right)}{\Gamma(s)}
$$

to evaluate the integral of product $K$-Bessel functions:

$$
\int_{0}^{\infty} K_{\frac{k}{2}-\frac{n}{4}+\frac{i n t}{2}}\left(2 \pi y_{n-k}\right) K_{\frac{k}{2}-\frac{n}{4}-\frac{i n t}{2}}\left(2 \pi y_{n-k}\right) y_{n-k}^{\sum_{j=1}^{n-1} b_{n-k, j} \nu_{j}+\frac{n}{2}-k(n-k)} \frac{d y_{n-k}}{y_{n-k}} .
$$


These $\gamma$ factors will form completed $\zeta$-functions:

$$
\begin{aligned}
& \frac{1}{8} \frac{\xi\left((1-k)(n-k)+\sum_{j=1}^{n-1} b_{n-k, j} \nu_{j}\right) \xi\left((1-k)(n-k)+\sum_{j=1}^{n-1} b_{n-k, j} \nu_{j}-\frac{n}{2}+k+i n t\right)}{\xi\left(2(1-k)(n-k)+2 \sum_{j=1}^{n-1} b_{n-k, j} \nu_{j}-n+2 k\right)} \\
& \times \xi\left((1-k)(n-k)+\sum_{j=1}^{n-1} b_{n-k, j} \nu_{j}-\frac{n}{2}+k-i n t\right) \xi\left((1-k)(n-k)+\sum_{j=1}^{n-1} b_{n-k, j} \nu_{j}-n+2 k\right) .
\end{aligned}
$$

Now we gather $y_{i}$ factors for $i \neq n-k$ :

$$
\begin{aligned}
& \frac{\left(\prod_{\substack{i=1 \\
i \neq n-k}}^{n-1} y_{i}^{\sum_{j=1}^{n-1} b_{i, j} \nu_{j}}\right)\left(y_{1} y_{2}^{2} \cdots y_{n-k-1}^{n-k-1}\right)\left(y_{n-(k-1)}^{k-1} \cdots y_{n-1}\right)}{\left(y_{(n-k)+1}^{k-h-2} \cdots y_{(n-k)+(k-h-2)^{+}}\right) \prod_{\substack{\ell=1 \\
\ell \neq n-k}}^{n-1} y_{\ell}^{\ell(n-\ell)}} \\
& \times\left(y_{(n-k)+1} \cdots y_{(n-k)+(k-h-1)^{+}}\right)^{-\sum_{j=1}^{n-1} b_{n-k, j} \nu_{j}+k(n-k)-h-1} \\
& =\prod_{i=1}^{n-k-1} y_{i}^{\sum_{j=1}^{n-1} b_{i, j} \nu_{j}+i-i(n-i)} \prod_{i=n-k+1}^{n-h-1} y_{i}^{k(n-k)-i(n-i)+\sum_{j=1}^{n-1}\left(b_{i, j}-b_{n-k, j}\right) \nu_{j}} \prod_{i=n-h}^{n-1} y_{i}^{\sum_{j=1}^{n-1} b_{i, j} \nu_{j}+n-i-i(n-i)} \text {. }
\end{aligned}
$$

Now we put everything together,

$$
\begin{aligned}
\Omega_{k}= & \frac{2}{\left|\xi\left(\frac{n}{2}+i n t\right)\right|^{2}} \int_{\left(\mathbb{R}^{+}\right)^{n-2}} \frac{1}{(2 \pi i)^{n-1}} \int_{(2)} \cdots \int_{(2)} \tilde{\eta}(\nu) \\
& \times \sum_{h=0}^{k-1} \frac{\xi\left((1-k)(n-k)+\sum_{j=1}^{n-1} b_{n-k, j} \nu_{j}\right) \xi\left((1-k)(n-k)+\sum_{j=1}^{n-1} b_{n-k, j} \nu_{j}-\frac{n}{2}+k+i n t\right)}{\xi\left(2(1-k)(n-k)+2 \sum_{j=1}^{n-1} b_{n-k, j} \nu_{j}-n+2 k\right)} \\
& \times \xi\left((1-k)(n-k)+\sum_{j=1}^{n-1} b_{n-k, j} \nu_{j}-\frac{n}{2}+k-i n t\right) \xi\left(\sum_{j=1}^{n-1} b_{n-k, j} \nu_{j}-k(n-k)+h+1\right) \\
& \times \prod_{i=1}^{n-k-1} y_{i}^{\sum_{j=1}^{n-1} b_{i, j} \nu_{j}+i-i(n-i)} \prod_{i=n-k+1}^{n-h-1} y_{i}^{k(n-k)-i(n-i)+\sum_{j=1}^{n-1}\left(b_{i, j}-b_{n-k, j}\right) \nu_{j}} \prod_{i=n-h}^{n-1} y_{i}^{\sum_{j=1}^{n-1} b_{i, j} \nu_{j}+n-i-i(n-i)} \\
& \prod_{\ell=1}^{n-1} d \nu_{\ell} \prod_{\substack{i=1 \\
i \neq n-k}}^{n-1} \frac{d y_{i}}{y_{i}}
\end{aligned}
$$


Combining the previous two lemmas, we let

$$
\begin{aligned}
\Omega_{k, h}:= & \frac{2}{\left|\xi\left(\frac{n}{2}+i n t\right)\right|^{2}} \int_{\left(\mathbb{R}^{+}\right)^{n-2}} \frac{1}{(2 \pi i)^{n-1}} \int_{(2)} \cdots \int_{(2)} \tilde{\eta}(\nu) \\
& \times \frac{\xi\left((1-k)(n-k)+\sum_{j=1}^{n-1} b_{n-k, j} \nu_{j}\right) \xi\left((1-k)(n-k)+\sum_{j=1}^{n-1} b_{n-k, j} \nu_{j}-\frac{n}{2}+k+i n t\right)}{\xi\left(2(1-k)(n-k)+2 \sum_{j=1}^{n-1} b_{n-k, j} \nu_{j}-n+2 k\right)} \\
& \times \xi\left((1-k)(n-k)+\sum_{j=1}^{n-1} b_{n-k, j} \nu_{j}-\frac{n}{2}+k-i n t\right) \xi\left(\sum_{j=1}^{n-1} b_{n-k, j} \nu_{j}-k(n-k)+h+1\right) \\
& \times \prod_{i=1}^{n-k-1} y_{i}^{\sum_{j=1}^{n-1} b_{i, j} \nu_{j}+i-i(n-i)} \prod_{i=n-k+1}^{n-h-1} y_{i}^{k(n-k)-i(n-i)+\sum_{j=1}^{n-1}\left(b_{i, j}-b_{n-k, j}\right) \nu_{j}} \prod_{i=n-h}^{n-1} y_{i}^{\sum_{j=1}^{n-1} b_{i, j} \nu_{j}+n-i-i(n-i)} \\
& \prod_{\ell=1}^{n-1} d \nu_{\ell} \prod_{\substack{i=1 \\
i \neq n-k}}^{n-1} \frac{d y_{i}}{y_{i}}
\end{aligned}
$$

for $1 \leq k \leq n-1$ and $0 \leq h \leq k-1$, then we claim that the main term comes from $\Omega_{n-1,0}$. We have

$$
\begin{aligned}
\Omega_{n-1,0}= & \frac{2}{\left|\xi\left(\frac{n}{2}+i n t\right)\right|^{2}} \int_{\left(\mathbb{R}^{+}\right)^{n-2}} \frac{1}{(2 \pi i)^{n-1}} \int_{(2)} \cdots \int_{(2)} \tilde{\eta}(\nu) \\
& \times \frac{\xi\left(2-n+\sum_{j=1}^{n-1} b_{1, j} \nu_{j}\right)^{2} \xi\left(1-\frac{n}{2}+\sum_{j=1}^{n-1} b_{n-k, j} \nu_{j}+i n t\right) \xi\left(1-\frac{n}{2}+\sum_{j=1}^{n-1} b_{n-k, j} \nu_{j}-i n t\right)}{\xi\left(2-n+2 \sum_{j=1}^{n-1} b_{n-k, j} \nu_{j}\right)} \\
& \times \prod_{i=2}^{n-1} y_{i}^{n-1-i(n-i)+\sum_{j=1}^{n-1}\left(b_{i, j}-b_{1, j}\right) \nu_{j}} \prod_{\ell=1}^{n-1} d \nu_{\ell} \prod_{\substack{i=1 \\
i \neq n-k}}^{n-1} \frac{d y_{i}}{y_{i}}
\end{aligned}
$$

\section{Lemma 5.5.}

$$
\Omega_{n-1,0}=\frac{2 \tilde{\eta}\left(\frac{2}{n}, \ldots, \frac{2}{n}\right) \log t}{\xi(n) n^{n-2}}+O(1) .
$$

Proof. Notice that there are $n-2$ Mellin transforms and $n-1$ Mellin inversion in the above expression. Thus we can take advantage of the Mellin inversion theorem. We make the change of variables

$$
\sum_{j=1}^{n-1}\left(b_{i, j}-b_{1, j}\right) \nu_{j} \mapsto s_{i-1} \quad \text { for } 2 \leq i \leq n-1
$$

and $s_{n-1}=v_{n-1}$. The Jacobian is calculated to be $\frac{1}{n^{n-3}}$ and explicitly we have

$$
\begin{aligned}
v_{1} & =\frac{s_{1}-s_{n-3}+2 s_{n-2}}{n}+v_{n-1}, \\
v_{i} & =\frac{2 s_{n-i-1}-s_{n-i}-s_{n-i-2}}{n} \quad \text { for } 2 \leq i \leq n-3, \\
v_{n-2} & =\frac{2 s_{1}-s_{2}}{8} \\
v_{n-1} & =s_{n-1} .
\end{aligned}
$$


By 5.1, we also have $\sum_{j=1}^{n-1} b_{1, j} \nu_{j} \mapsto s_{1}+n s_{n-1}$. So

$$
\begin{aligned}
\Omega_{n-1,0}= & \frac{1}{n^{n-3}} \frac{2}{\left|\xi\left(\frac{n}{2}+i n t\right)\right|^{2}} \int_{\left(\mathbb{R}^{+}\right)^{n-2}} \frac{1}{(2 \pi i)^{n-1}} \int_{\left(\sigma_{1}\right)} \ldots \int_{\left(\sigma_{n-1}\right)} \\
& \tilde{\eta}\left(\frac{s_{1}-s_{n-3}+2 s_{n-2}}{n}+s_{n-1}, \ldots, \frac{2 s_{n-i-1}-s_{n-i}-s_{n-i-2}}{n}, \ldots, \frac{2 s_{1}-s_{2}}{n}, s_{n-1}\right) \\
& \times \frac{\xi\left(2-n+s_{1}+n s_{n-1}\right)^{2} \xi\left(1-\frac{n}{2}+s_{1}+n s_{n-1}+i n t\right) \xi\left(1-\frac{n}{2}+s_{1}+n s_{n-1}-i n t\right)}{\xi\left(2-n+2 s_{1}+2 n s_{n-1}\right)} \\
& \times \prod_{i=2}^{n-1} y_{i}^{n-1-i(n-i)+s_{i-1}} \prod_{\ell=1}^{n-1} d s_{\ell} \prod_{i=1}^{n-1} \frac{d y_{i}}{y_{i}} \\
= & \frac{1}{n^{n-3}} \frac{2}{\left|\xi\left(\frac{n}{2}+i n t\right)\right|^{2}} \frac{1}{2 \pi i} \int_{\left(\sigma_{n-1}\right)} \tilde{\eta}\left(s_{n-1}, \frac{2}{n}, \ldots, \frac{2}{n}, s_{n-1}\right) \\
& \times \frac{\xi\left(n s_{n-1}-1\right)^{2} \xi\left(\frac{n}{2}-2+n s_{n-1}+i n t\right) \xi\left(\frac{n}{2}-2+n s_{n-1}-i n t\right)}{\xi\left(n-4+2 n s_{n-1}\right)} d s_{n-1}
\end{aligned}
$$

We now shift contour from $\sigma_{n-1}$ to $\frac{2-\Delta}{n}$ for some small $\Delta>0$. In the process, we pick up the residue corresponding to the pole of the $\zeta\left(n s_{n-1}-1\right)^{2}$ term at $s_{n-1}=\frac{2}{n}$. Hence

$$
\Omega_{n-1,0}=R_{1}+R_{2}
$$

where

$$
\begin{aligned}
R_{1} \quad & :=\frac{2 \tilde{\eta}\left(\frac{2}{n}, \ldots, \frac{2}{n}\right)}{\xi(n) n^{n-1}}\left(O(1)+\frac{n}{2}\left(\frac{\Gamma^{\prime}}{\Gamma}\left(\frac{n}{4}+\frac{i n t}{2}\right)+\frac{\Gamma^{\prime}}{\Gamma}\left(\frac{n}{4}-\frac{i n t}{2}\right)\right)\right) \\
& =\frac{2 \tilde{\eta}\left(\frac{2}{n}, \ldots, \frac{2}{n}\right) \log t}{\xi(n) n^{n-2}}+O(1) \\
R_{2}:= & \frac{1}{n^{n-3}} \frac{2}{\left|\xi\left(\frac{n}{2}+i n t\right)\right|^{2}} \frac{1}{2 \pi i} \int_{\left(\frac{2-\Delta}{n}\right)} \tilde{\eta}\left(s_{n-1}, \frac{2}{n}, \ldots, \frac{2}{n}, s_{n-1}\right) \\
& \times \frac{\xi\left(n s_{n-1}-1\right)^{2} \xi\left(\frac{n}{2}-2+n s_{n-1}+i n t\right) \xi\left(\frac{n}{2}-2+n s_{n-1}-i n t\right)}{\xi\left(n-4+2 n s_{n-1}\right)} d s_{n-1} .
\end{aligned}
$$

Let $f\left(t_{2}\right)=\tilde{\eta}\left(\frac{2-\Delta}{n}+i t_{2}, \frac{2}{n}, \ldots, \frac{2}{n}, \frac{2-\Delta}{n}+i t_{2}\right)$, then $f\left(t_{s}\right)$ is a function with rapid decay in $t_{2}$. We can bound the $R_{2}$ integral as follows:

$$
\begin{aligned}
R_{2} \ll & \int_{-\infty}^{\infty}\left|f\left(t_{2}\right)\right| \frac{\left|\zeta\left(1-\Delta+i n t_{2}\right)\right|^{2}\left|\Gamma\left(\frac{1-\Delta}{2}+\frac{i n t_{2}}{2}\right)\right|^{2}}{\zeta\left(n-2 \Delta+2 i n t_{2}\right) \Gamma\left(\frac{n}{2}-\Delta+i n t_{2}\right)} \\
& \times \frac{\left|\zeta\left(\frac{n}{2}-\Delta+i n\left(t_{2}+t\right)\right)\right|\left|\zeta\left(\frac{n}{2}-\Delta+i n\left(t_{2}-t\right)\right)\right|\left|\Gamma\left(\frac{n}{4}-\frac{\Delta}{2}+\frac{i n\left(t_{2}+t\right)}{2}\right)\right|\left|\Gamma\left(\frac{n}{4}-\frac{\Delta}{2}+\frac{i n\left(t_{2}-t\right)}{2}\right)\right|}{\left|\zeta\left(\frac{n}{2}+i n t\right)\right|^{2}\left|\Gamma\left(\frac{n}{4}+\frac{i n t}{2}\right)\right|^{2}} d t_{2}
\end{aligned}
$$


By Stirling's formula,

$$
\begin{aligned}
& \frac{\left|\Gamma\left(\frac{n}{4}-\frac{\Delta}{2}+\frac{i n\left(t_{2}+t\right)}{2}\right)\right|\left|\Gamma\left(\frac{n}{4}-\frac{\Delta}{2}+\frac{i n\left(t_{2}-t\right)}{2}\right)\right|}{\left|\Gamma\left(\frac{n}{4}+\frac{i n t}{2}\right)\right|^{2}} \\
\ll & \frac{e^{-\frac{\pi n}{4}\left(\left|t_{2}+t\right|+\left|t_{2}-t\right|\right)}\left|t_{2}+t\right|^{\frac{n}{4}-\frac{1}{2}-\frac{\Delta}{2}}\left|t_{2}-t\right|^{\frac{n}{4}-\frac{1}{2}-\frac{\Delta}{2}}}{e^{-\frac{\pi n t}{2}}|t|^{\frac{n}{2}-1}} \\
\ll & \frac{\left|t_{2}+t\right|^{\frac{n}{4}-\frac{1}{2}-\frac{\Delta}{2}}\left|t_{2}-t\right|^{\frac{n}{4}-\frac{1}{2}-\frac{\Delta}{2}}}{|t|^{\frac{n}{2}-1}} .
\end{aligned}
$$

So

$$
R_{2} \ll t^{-\Delta}
$$

Now we tackle $\Omega_{k, h}$ for general $k, h$.

Proposition 5.2. For $(k, h) \neq(n-1,0)$, we have

$$
\Omega_{k, h}=O(1) .
$$

Proof. We make the convenient change of variable

$$
\left(\begin{array}{c}
s_{1} \\
s_{2} \\
\vdots \\
s_{n-1}
\end{array}\right)=M_{k, h} \cdot\left(b_{i, j}\right) \cdot\left(\begin{array}{c}
v_{1} \\
v_{2} \\
\vdots \\
v_{n-1}
\end{array}\right)
$$

where the matrix $M_{k, h}$ is the identity matrix except for the entries $(j, n-k)$ being -1 for $n-k+1 \leq j \leq$ $n-h-1$. With this change variable we have

$$
\begin{aligned}
\Omega_{k, h}= & \frac{2}{n^{n-2}\left|\xi\left(\frac{n}{2}+i n t\right)\right|^{2}} \int_{\left(\mathbb{R}^{+}\right)^{n-2}} \frac{1}{(2 \pi i)^{n-1}} \int_{\left(\sigma_{1}\right)} \ldots \int_{\left(\sigma_{n-1}\right)} \tilde{\eta}\left(v_{1}, \ldots, v_{n-1}\right) \\
& \times \frac{\xi\left((1-k)(n-k)+s_{n-k}\right) \xi\left(s_{n-k}-k(n-k)+h+1\right)}{\xi\left(2(1-k)(n-k)+2 s_{n-k}-n+2 k\right)} \\
& \times \xi\left((1-k)(n-k)+s_{n-k}-\frac{n}{2}+k-i n t\right) \xi\left((1-k)(n-k)+s_{n-k}-\frac{n}{2}+k+i n t\right) \\
& \times \prod_{i=1}^{n-k-1} y_{i}^{s_{i}+i-i(n-i)} \prod_{i=n-k+1}^{n-h-1} y_{i}^{k(n-k)-i(n-i)+s_{i}} \prod_{i=n-h}^{n-1} y_{i}^{s_{i}+n-i-i(n-i)} \prod_{\ell=1}^{n-1} d s_{\ell} \prod_{i=1}^{n-1} \frac{d y_{i}}{y_{i}} \\
= & \frac{1 \neq n-k}{n^{n-2}\left|\xi\left(\frac{n}{2}+i n t\right)\right|^{2}} \frac{1}{2 \pi i} \int_{\left(\sigma_{n-1}\right)} f\left(s_{n-1}\right) \frac{\xi\left((1-k)(n-k)+s_{n-k}\right) \xi\left(s_{n-k}-k(n-k)+h+1\right)}{\xi\left(2(1-k)(n-k)+2 s_{n-k}-n+2 k\right)} \\
& \times \xi\left((1-k)(n-k)+s_{n-k}-\frac{n}{2}+k-i n t\right) \xi\left((1-k)(n-k)+s_{n-k}-\frac{n}{2}+k+i n t\right) d s_{n-1} .
\end{aligned}
$$

Now we will shift contours to $\operatorname{Re}\left(s_{n-k}\right)=1-(1-k)(n-k)-\Delta$ for small $\Delta>0$. There are two cases to consider.

Case 1: $n-k=h+1$ and $k \neq n-1$.

In this situation, $\xi\left((1-k)(n-k)+s_{n-k}\right)=\xi\left(s_{n-k}-k(n-k)+h+1\right)$. As a result, we will encounter a pole of order 2 during the contour shift at $s_{n-1}=1-(1-k)(n-k)$. So

$$
\Omega_{k, h}=R_{1}+R_{2}
$$


where

$$
R_{1}=\frac{C_{1} \xi\left(k+1-\frac{n}{2}\right) \xi\left(k+1-\frac{n}{2}\right)}{\left|\xi\left(\frac{n}{2}+i n t\right)\right|^{2}}\left(C_{2}+\frac{\xi^{\prime}}{\xi}\left(1+k-\frac{n}{2}+i n t\right)+\frac{\xi^{\prime}}{\xi}\left(1+k-\frac{n}{2}-i n t\right)\right)
$$

and

$$
\begin{aligned}
R_{2}= & \frac{2}{n^{n-2}\left|\xi\left(\frac{n}{2}+i n t\right)\right|^{2}} \frac{1}{2 \pi i} \int_{(1-(1-k)(n-k)-\Delta)} f\left(s_{n-1}\right) \frac{\xi\left((1-k)(n-k)+s_{n-k}\right)^{2}}{\xi\left(2(1-k)(n-k)+2 s_{n-k}-n+2 k\right)} \\
& \times \xi\left((1-k)(n-k)+s_{n-k}-\frac{n}{2}+k-i n t\right) \xi\left((1-k)(n-k)+s_{n-k}-\frac{n}{2}+k+i n t\right) d s_{n-1} .
\end{aligned}
$$

By Stirling's formula, one can show that

$$
R_{1}+R_{2} \ll t^{-\frac{1}{2}}
$$

Case 2: $n-k \neq h+1$.

For this case, $\xi\left((1-k)(n-k)+s_{n-k}\right) \neq \xi\left(s_{n-k}-k(n-k)+h+1\right)$. As a result, we will encounter a pole of order 1 during the contour shift at $s_{n-1}=1-(1-k)(n-k)$. So

$$
\Omega_{k, h}=R_{3}+R_{4}
$$

where

$$
R_{3}=C_{3} \frac{\xi\left(1-\frac{n}{2}+k+i n t\right) \xi\left(1-\frac{n}{2}+k-i n t\right)}{\left|\xi\left(\frac{n}{2}+i n t\right)\right|^{2}}
$$

and

$$
\begin{aligned}
R_{4}= & \frac{2}{n^{n-2}\left|\xi\left(\frac{n}{2}+i n t\right)\right|^{2}} \frac{1}{2 \pi i} \int_{(1-(1-k)(n-k)-\Delta)} f\left(s_{n-1}\right) \frac{\xi\left((1-k)(n-k)+s_{n-k}\right) \xi\left(s_{n-k}-k(n-k)+h+1\right)}{\xi\left(2(1-k)(n-k)+2 s_{n-k}-n+2 k\right)} \\
& \times \xi\left((1-k)(n-k)+s_{n-k}-\frac{n}{2}+k-i n t\right) \xi\left((1-k)(n-k)+s_{n-k}-\frac{n}{2}+k+i n t\right) d s_{n-1} .
\end{aligned}
$$

By Stirling's formula, one can show that

$$
R_{3}+R_{4}=O(1)
$$




\section{REFERENCES}

[Ar] J. Arthur, A trace formula for reductive groups. II. Applications of a truncation operator, Compos. Math.40 (1980), 87-121

[B] D. Bump, Automorphic Forms on $G L(3, \mathbb{R})$, Lecture Notes in Mathematics 1083, Springer-Verlag (1984)

[Bu] L.A. Bunimovich, On the Ergodic Properties of Nowhere Dispersing Billiards, Commun Math Phys. 65 (1979) 295312 .

[BS] L.A. Bunimovich, Ya. G. Sinai Markov Partitions for Dispersed Billiards. Commun Math Phys. 78 (1980) $247-280$.

[Co] Y. Colin de Verdiére, Ergodicité et fonctions propres du laplacien, Com. Math. Phys., 102(1985), 497-502.

[Go] D. Goldfeld, Automorphic forms and L-functions for the group $G L(n, \mathbb{R})$, Cambridge studies in advanced mathematics 99 (2006)

[GR] I. S. Gradshteyn, I. M. Ryzhik, Table of Integrals, Series, and Products, seventh edition, Academic Press, Elsevier (2007)

[HR] D.A. Hejhal, D. Rackner, On the topography of Maass wave forms, Exper. Math. 1(1992), $275-305$.

[IT] K. Imai and A. Terras, The Fourier Expansions of Eisenstein Series for GL(3, Z), Trans. AMS 273 (1982), \#2, 679-694

[I] H. Iwaniec, Spectral Methods of Automorphic Forms, Graduate Studies in Mathematics 53, American Mathematical Society (2002)

[L] R. P. Langlands, On the functional equations satisfied by Eisenstein series, Lecture Notes in Mathematics, 544, Springer-Verlag, Berlin-New York, (1976)

[Li] E. Lindenstrauss, Invariant measures and arithmetic quantum unique ergodicity, Ann. of Math. (2) 163 (2006), 165-219.

[LS] W. Luo, P. Sarnak, Quantum Ergodicity of Eigenfunctions on PSL $(\mathbb{Z}) \backslash \mathbb{H}^{2}$, Inst. Hautes Etudes Sci. Publ. Math. 81 (1995), 207-237

[MW] C. Moeglin, J.-L. Waldspurger, Spectral decomposition and Eisenstein series, Cambridge University Press, 113 (1995)

[PK] R.B. Paris, D. Kaminski, Asymptotics and Mellin-Barnes Integrals, Encyclopedia of Mathematics and Its Applications, 85 (2001)

[Ra] S. Ramanujan, Some formulae in the arithmetic theory of numbers, Messenger of Math., 45 (1916), 81-84.

[RS] Z. Rudnick, P. Sarnak, The behavior of eigenstates of arithmetic hyperbolic manifolds, Com. Math. Phys., 161 (1994), 195-213.

[S] K. Soundararajan, Quantum unique ergodicity for $S L_{2}(\mathbb{Z}) \backslash \mathbb{H}$. Ann. of Math. (2) 172 (2010), no. 2, $1529-1538$.

[Sh] A. I. Schnirelman, Ergodic properties of eigenfunctions, Usp. Math. Nauk., 29(1974), 79-98

[SV1] L. Silberman, A. Venkatesh, On quantum unique ergodicity for locally symmetric spaces. Geom. Funct. Anal. 17 (2007), no. 3, 960-998.

[SV2] L. Silberman, A. Venkatesh, Entropy bounds and quantum unique ergodicity for Hecke eigenfunctions on division algebras. https://arxiv.org/abs/1606.02267

[Ve] A. Venkov, The Selberg trace formula for $S L(3, \mathbb{Z})$ Dokl. Akad. Nauk SSSR 228 (1976), No. 2, 273-276

[Ze1] S. Zelditch, Uniform distribution of eigenfunctions on compact hyperbolic surfaces, Duke Math. Jnl., 55(1987), 919941

[Ze2] S. Zelditch, Selberg trace formulae and equidistribution theorems, Memoirs of AMS, Vol.96, No.465, 1992.

10 Hillhouse Ave. New Haven, CT 06511

E-mail address: liyang.zhang@yale.edu 